\title{
Synthesis of 1,2,3-triazole-containing furanosyl nucleoside analogs and their phosphate, phosphoramidate or phoshonate derivatives as potential sugar diphosphate or nucleotide mimetics
}

\author{
Andreia Fortuna, ${ }^{a, b}$ Paulo J. Costa, ${ }^{b}$ M. Fátima M. Piedade, ${ }^{a, c}$ M. Conceição Oliveira ${ }^{c}$ \\ and Nuno M. Xavier ${ }^{\mathrm{a}, *}$ \\ ${ }^{a}$ Centro de Química Estrutural, Faculdade de Ciências, Universidade de Lisboa, \\ Ed. C8, $5^{\circ}$ Piso, Campo Grande, 1749-016 Lisboa, Portugal \\ ${ }^{b}$ University of Lisboa, Faculty of Sciences, BioISI - Biosystems \& Integrative Sciences \\ Institute, Campo Grande, C8 bdg, 1749-016 Lisboa, Portugal \\ ${ }^{c}$ Centro de Química Estrutural, Instituto Superior Técnico, Universidade de Lisboa, \\ Av. Rovisco Pais, 1049-001 Lisboa, Portugal \\ *corresponding author, e-mail: nmxavier@fc.ul.pt
}

\begin{abstract}
The synthesis of a variety of novel, rather stable and potentially bioactive nucleoside analogs and nucleotide mimetics based on xylofuranose scaffolds and comprising a 1,2,3-triazole moiety as a surrogate for a nucleobase or a phosphate group is reported. Isonucleosides embodying a 3-O-(benzyltriazolyl)methyl moiety at $\mathrm{C}-3$ were accessed by using the $\mathrm{Cu}(\mathrm{I})$-catalyzed "click" 1,3-dipolar cycloaddition between 3-O-propargyl1,2- $O$-isopropylidene- $\alpha$-D-xylofuranose and benzyl azide as the key step. Related isonucleotides comprising a phosphate or a phosphoramidate moiety at C-5 were obtained via 5-O-phosphorylation of acetonide-protected 3-O-propargyl xylofuranose followed by "click" cycloaddition or by Staudinger reaction of a 5'-azido $N$ benzyltriazole isonucleoside with triethyl phosphite, respectively. Hydroxy, amino- or bromomethyl triazole $5^{\prime}$-isonucleosides were synthesized through thermal cycloaddition between 5-azido 3-O-benzyl/dodecyl-1,2- $O$-isopropylidene- $\alpha$-D-xylofuranoses and propargyl alcohol, propargylamine or propargyl bromide, respectively. The regiochemical outcome of the cycloaddition reactions was influenced by nature of the alkyne hetero substituent (alkyne $\mathrm{CH}_{2} \mathrm{X}$ substituent). The $5^{\prime}$-isonucleosides were converted into their [(xylofuranos-5-yl)triazolyl]methyl phosphate, phosphoramidate and phosphonate derivatives as prospective sugar diphosphate mimetics by an appropriate method involving treatment with diethyl phosphorochloridate or a Michaelis-Arbuzov reaction. 4-Phosphonomethyl-1-xylofuranos-5'-yl triazoles were converted into 1,2-O-acetyl glycosyl donors and subsequently subjected to nucleosidation with uracil leading to the corresponding uracil nucleoside 5'(triazolyl)methyl phosphonates, whose structure potentially mimics that of a nucleoside diphosphate.
\end{abstract}




\section{Introduction}

Nucleosides and nucleotides are key molecules in essential biological events including nucleic acid synthesis, cell division, cell signalling, and metabolism. The development of synthetic analogues of these molecules, aiming at inhibiting nucleotide-dependent processes/enzymes that are overactivated and play crucial roles in diseases such as cancer or viral infections, has been explored throughout the years. A significant number of nucleoside and nucleotide analogs have reached clinical application as chemotherapeutic agents, acting mostly as nucleic acid antimetabolites, from which cytarabine, gemcitabine or azidothymidine can be highlighted. ${ }^{1}$ Their mechanism of action involves a kinase-mediated conversion into di- and triphosphate nucleoside metabolites and further incorporation into nucleic acids or altarnatively, inhibition of polymerases or other enzymes involved in nucleic acid or nucleotide biosynthesis, inducing inhibition of cell division or viral replication. ${ }^{1}$ Despite the proven anticancer and antiviral efficacy of nucleos(t)ide analogs, some drawbacks are associated with their use, including their high polarity which results in low oral bioavailability and the intrinsic or acquired resistance that cancer or virus-infected cells exert against their action. Among these mechanisms are the downregulation of nucleoside transporters, ${ }^{2}$ hindering their passage to the intracellular medium, and of nucleoside kinases, ${ }^{3}$ which results in a decreased formation of nucleoside phosphates. The intracellular levels of the former metabolites are also reduced by their increased dephosphorylation due to the overexpression of nucleotidases as another relevant nucleoside-specific mechanism of chemotherapy resistance. ${ }^{4}$ Nucleoside analogs or mimetics possessing structural features that may allow them to overcome these issues have been synthesized. Modifications at the nucleobase, sugar moiety or the insertion of phosphate group analogs have been performed aiming at nucleos(t)ide analogs of improved bioavailability, cell-penetrating ability or stability to enzymatic hydrolysis. These molecules include acyclic nucleos(t)ides, ${ }^{5}$ carbocyclic derivatives $^{6}$ or other sugar heteroanalog-containing nucleosides, ${ }^{7}$ nucleosides comprising uncommon glycosyl units, ${ }^{8} C$-nucleosides, ${ }^{9}$ nucleoside alkyl/aryl phosphodi/triesters, ${ }^{10}$ phosphoramidates or H- or C-phosphonates. ${ }^{10,11}$ Other biological effects of therapeutic relevance beyond anticancer and antiviral activities have also been reported for nucleos(t)ide analogs. Various naturally-occurring nucleosides and synthetic derivatives were described as possessing antibiotic properties, acting as inhibitors of crucial microbial cell processes such as cell wall assembly, nucleotide metabolism, nucleic acid or protein biosynthesis. ${ }^{12}$ Some reports, which include studies previously undertaken by us, revealed the ability of nucleoside analogs to inhibit cholinesterases, ${ }^{13}$ which are important therapeutic targets for Alzheimer's disease symptomatic treatment. Among the latter compounds are $5^{\prime} / 6^{\prime}$-isonucleosides, ${ }^{13 \mathrm{~d}-\mathrm{f}}$ which comprise a nucleobase or an analogous nitrogeneous heteroaromatic moiety $N$-linked to a sugar moiety at C-5 or C6 , and D-glucofuranuronamide-based $N$-glycosyl triazoles. ${ }^{13 e}$ Isonucleosides are rather stable nucleoside regioisomers since their $\mathrm{N}-\mathrm{C}$ bond linking the sugar and nucleobase 
units is less susceptible to chemical or enzymatic hydrolysis than the $\mathrm{N}$-glycosidic bond present in nucleosides. Furanosyl $2^{\prime} / 3^{\prime}$-isonucleosides displaying anticancer ${ }^{14}$ and antiviral $^{15}$ properties were reported.

The biological efficacy and broad bioactivity profile of nucleosides and nucleotide analogs encourage the development of new structures that may circumvent the limitations of the clinically-used ones, exhibit alternative mechanisms of action, and offer new therapeutic opportunities. In this context, we report herein on the synthesis of a variety of xylofuranose-based isonucleosides $(\mathbf{A}, \mathbf{B})$ together with mimetics of sugar diphosphate (D) and nucleotides (C, E) comprising a 1,2,3-triazole moiety (Fig. 1). The broad biological profile associated with this motif, ${ }^{16}$ its ability for hydrogen-bond, iondipole, cation- $\pi$ or $\pi$-stacking interactions with amino acid residues or to coordinate metals, enabling molecular recognition by enzymes or receptors and its high chemical and enzymatic stability are relevant aspects that motivate the inclusion of this unit in new potentially bioactive structures. Furthermore, this moiety can be accessed by a straightforward synthetic methodology based on an Huisgen azide-alkyne cycloaddition reaction. ${ }^{17}$ Reported examples on furanosyl nucleoside analogs containing a 1,2,3triazole moiety ${ }^{17 \mathrm{~b}}$ revealed their propensity to exhibit antiviral, ${ }^{18}$ anticancer ${ }^{19}$ or antifungal activities, ${ }^{20}$ and to inhibit nucleotide-dependent enzymes. ${ }^{21}$ This $\mathrm{N}$ heteroaromatic system was included as a surrogate of a nucleobase in isonucleosides of types $\mathbf{A}$ and related isonucleotides $\mathbf{C}$, comprising a benzyltriazole motif linked by an oxymethylene spacer to the xylofuranose unit at C-3 (3'-O-methyl-branched isonucleosides), and in 5'-isonucleosides B containing hydroxy, amino- or bromomethyl triazole systems. A terminal aminomethyl triazole isonucleoside was previously reported as a moderate acetylcholinesterase inhibitor, ${ }^{13 \mathrm{c}}$ which shows the biological interest of this type of structure.

Moreover, the triazole unit was also aimed as a potential neutral and rather stable surrogate of a phosphate group in compounds of types $\mathbf{D}$ and E. Such bioisostere replacement was already demonstrated to be feasible in the mimicry of glycosyl phosphates, ${ }^{22}$ DNA oligonucleotides, ${ }^{23}$ nucleoside mono- or diphosphates, ${ }^{21 \mathrm{~d}}$ or nucleoside triphosphates. ${ }^{24}$ Non-charged phosphate group analogs such as di-Oethyl/phenyl phosphate, di- $O$-ethyl phosphoramidate or phosphonate moieties were installed in the molecules, which may solve polarity and low cell-penetrating ability concerns. Moreover, the di- $O$-alkyl/aryl phosph(on)ate and phosphoramidate moieties may undergo intracellular cleavage by phosphodiesterase-mediated hydrolysis, releasing the free phosph(on)ate and phosphoramidate isonucleosides, similarly to what occurs with ester-type nucleoside phosph(on)ate prodrugs. ${ }^{11 \mathrm{~b}}$ In compounds of types $\mathbf{D}$ and $\mathbf{E}$, the combination of a phosphate group analog with a triazole unit was intended to establish new potential neutral diphosphate mimetics of higher stability, namely the (triazolyl)methyl phosphate, phosphoramidate and phosphonate systems. The (triazolyl)methyl phosphonate fragment may render the molecules particularly stable due to their non-hydrolytically cleavable P-C bond, which encouraged the synthesis of 
prospective nucleoside diphosphate mimetics of type $\mathbf{E}$. To confer the molecules higher lipophilicity, and thus enhance their membrane permeability and bioavailability, $O$ benzyl and $O$-dodecyl groups were included in their sugar moieties.

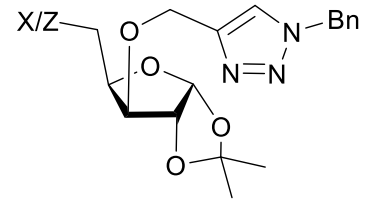

A $\mathrm{X}=\mathrm{OH}, \mathrm{N}_{3}$

$\stackrel{\mathrm{O}}{\|} \stackrel{\mathrm{O}}{\|} \underset{\mathrm{OP}}{\|}(\mathrm{OR})_{2}, \mathrm{NHP}(\mathrm{OEt})_{2}$

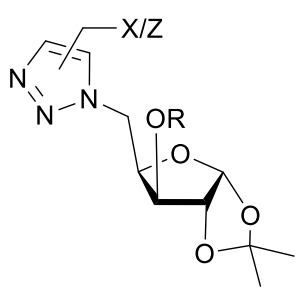

B $\mathrm{X}=\mathrm{OH}, \mathrm{NH}_{2}, \mathrm{Br}$

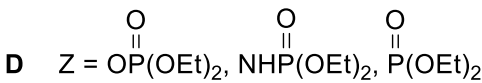

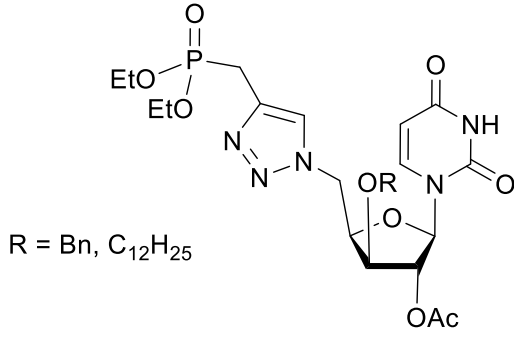

E

Fig. 1. General skeletons of triazole-containing isonucleosides $(\mathbf{A}, \mathbf{B})$, isonucleotides (C), sugar diphosphate mimetics (D) and nucleotide mimetics (D) exploited herein.

\section{Results and Discussion}

The synthesis of isonucleosides and related isonucleotides comprising an $O-(N-$ benzyltriazolyl)methyl system at a pentoxylofuranosyl template at C-3 (compounds of types $\mathrm{A}$ and $\mathrm{C}$, Fig. 1) involved the access to 3-O-propagyl xylofuranosyl derivatives as key synthons. Thus, diacetone-D-glucose (1, Scheme 1) was subjected to $O$ propargylation with propargyl bromide in the presence of sodium hydride, which was followed by removal of the primary acetonide by mild acid-mediated hydrolysis $(75 \%$ acetic acid aqueous soln.) to furnish the 5,6-diol 2. Oxidative cleavage of 2 with sodium periodate and subsequent reduction of the formed dialdofuranose with sodium borohydride afforded the 3-O-propargyl xylofuranose 3. "Click" 1,3-dipolar cycloaddition between $\mathbf{3}$ and benzyl azide in the presence of a copper iodide/Amberlyste A-21 catalytic system provided the $N$-benzyl triazole derivative 4 in quantitative yield. The synthesis of C-5 functionalized derivatives of this isonucleoside was then exploited. In order to increase its lipophilicity, a dodecyl moiety was introduced at $\mathrm{OH}-5$ through alkylation with dodecyl bromide in the presence of sodium hydride, affording 5. Phosphorylation of the 3-O-propargylated derivative 3 with diethyl phosphorochloridate in the presence of trimethylamine and 4-dimethylaminopyridine (DMAP) gave the corresponding phosphate triester 6, which was subsequently subjected to $\mathrm{Cu}(\mathrm{I})$ catalyzed cycloaddition with benzyl azide to provide the triazole-containing isonucleoside 5'-diethyl phosphate 7 in $92 \%$ yield. On the other hand, treatment of $\mathbf{3}$ with diphenylphosphoryl azide in the presence of 1,8-diazabicyclo[5.4.0]undec-7-ene (DBU) in toluene at $40{ }^{\circ} \mathrm{C}$ afforded the 5-O-diphenylphosphono xylofuranose derivative 
8. Even at a prolonged reaction time (16 h), no formation of a secondary product resulting from the nucleophilic replacement of the phosphate group by the azide anion occurred, as reported for this methodology, ${ }^{25}$ being the xylofuranos-5-yl diphenyl phosphate $\mathbf{8}$ obtained as the sole product in $68 \%$ yield. Further "click" cycloaddition with benzyl azide furnished the 5'-diphenyl phosphate isonucleoside 9 in $74 \%$ yield, as a diphenyl counterpart of isonucleotide 7 .

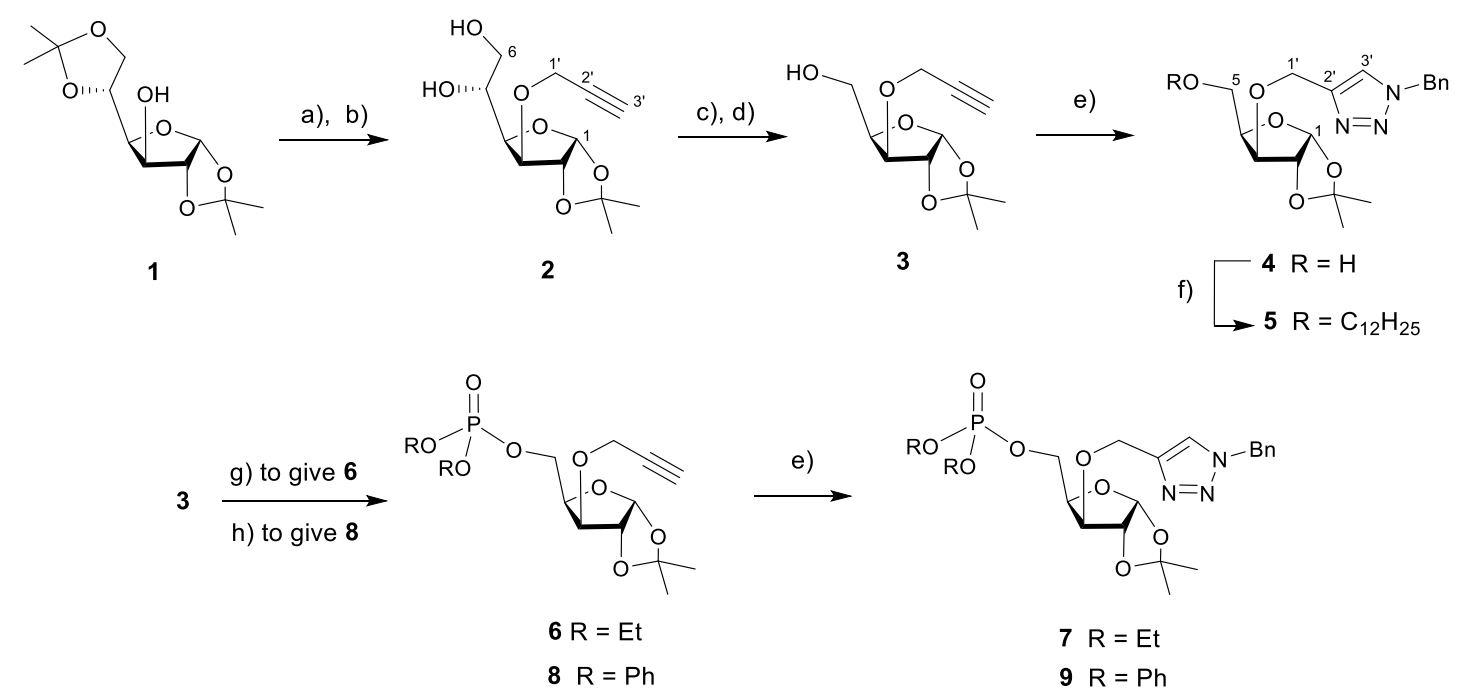

Scheme 1. Reagents and conditions: (a) $\mathrm{C}_{3} \mathrm{H}_{3} \mathrm{Br}, \mathrm{NaH}$, DMF, r. t., 16 h; (b) $\mathrm{AcOH}(75 \%$ aq. soln.), r. t. 16 h., 96\%, 2 steps. (c) $\mathrm{NaIO}_{4}, \mathrm{THF} / \mathrm{H}_{2} \mathrm{O}$ (3:1), r. t. 4 h; (d) $\mathrm{NaBH}_{4}$, $\mathrm{EtOH} / \mathrm{H}_{2} \mathrm{O}(2: 1)$, r. t., 1 h, 57\%, 2 steps; (e) $\mathrm{BnN}_{3}, \mathrm{CuI} / \mathrm{A}-21, \mathrm{CH}_{2} \mathrm{Cl}_{2}$, r. t., 24 h, $98 \%$ (4), $92 \%$ (7), 74\% (9); (f) $\mathrm{C}_{12} \mathrm{H}_{25} \mathrm{Br}, \mathrm{NaH}, \mathrm{DMF}$, r. t., 16 h, 95\%; (g) $\mathrm{ClPO}(\mathrm{OEt})_{2}, \mathrm{NEt}_{3}$, DMAP, $\mathrm{CH}_{2} \mathrm{Cl}_{2}$, r. t., 16 h, 98\%; (h) DPPA, DBU, toluene, r. t., 24 h, then $40{ }^{\circ} \mathrm{C}, 16 \mathrm{~h}$, $68 \%$.

For the synthesis of an isonucleoside phosphoramidate analog of isonucleotide $\mathbf{7}$, the use of the 5-azido 3-O-propargyl xylofuranose derivative $\mathbf{1 1}$ as precursor was firstly attempted (Scheme 2). It was synthesized by 3-O-propargylation (propargyl bromide/sodium hydride) of 5-azido-5-deoxy-1,2- $O$-isopropylidene- $\alpha$-D-xylofuranose (10), ${ }^{26}$ which was completed within a considerable shorter reaction time $(5 \mathrm{~min})$ than that previously reported $(2 \mathrm{~h})$ under similar conditions. ${ }^{27}$ However, due to the presence of both alkynyl and azide moieties, this compound was found to be unstable at room temperature and in dichloromethane solution in the presence of the CuI/A-21 catalyst, envolved towards the fused tetracyclic 1,5-dissubstituted triazole 12-a, resulting from intramolecular 1,3-dipolar cycloaddition. The ${ }^{13} \mathrm{C}$ NMR signals for C-4 and C-5 of the triazole moiety at $132.2 \mathrm{ppm}$ and $135.2 \mathrm{ppm}$, respectively, were indicative of a 1,5disubstituted derivative, ${ }^{28}$ while the fused system in 12-a was confirmed by the HMBC correlations between protons $\mathrm{H}-5$ of the sugar moiety with $\mathrm{C}-5$ of the triazole unit and between the oxymethylenic protons and both C-4/C-5 of the triazole motif. The sole formation of 12-a was previously reported via an intramolecular thermal Huisgen 
cycloaddition of $\mathbf{1 1}$ in toluene at $100{ }^{\circ} \mathrm{C},{ }^{27,29}$ a protocol which in our hands led to 12-a in $67 \%$ yield. Although our NMR data for this compound were in conformity with those reported, ${ }^{27}$ no concordance was verified between our single crystal X-ray structure (A, Fig. 2) with the published structure (CSD refcode DAQJOV) which corresponds to the L-xylo-configured derivative (see Fig. S37, in the Supplementary Information). The crystal structure of 12-a obtained in this work corresponds to the D-xylo-configured molecule with a fused tetracyclic framework containing a 7-membered ring (1,4oxazepane) between the triazole motif and the xylofuranose ring, which adopts a C3endo conformation. The bond lengths and angles for the two enantiomers are analogous (see CIF file).

To gain insight into the observed experimental regioselectivity in the thermal cycloaddition, density functional theory (DFT) calculations (PBE1PBE/6-311G**) were performed on 1,5- and 1,4-disubstituted triazoles (12-a, 12-b) to investigate their relative stability. The three-dimensional structures of $\mathbf{1 2}$-a and $\mathbf{1 2}-\mathbf{b}$ were built using Pymol $^{30}$ and geometry optimization (B, C, Fig. 2) was performed using Gaussian $09^{31}$ (see Computational Details in the Exptl. Sect. for more informations). The DFToptimised structure of 12-a (B, Fig. 2) was compared with the X-ray structure (A, Fig. 2) and the agreement was excellent with a root-mean square deviation (RMSD) of atomic positions of $\sim 0.09 \AA$ (see Fig. S38, in the Supplementary Information). The calculated values for the enthalpy $(\Delta \mathrm{H})$ and free energy $(\Delta \mathrm{G})$ of the 1,4-disubstituted regioisomer (C, Fig. 2) in toluene are higher by ca. $55 \mathrm{kcal} / \mathrm{mol}$ relative to those obtained for the 1,5-regioisomer (B, Fig. 2), thereby supporting the observed regioselectivity and indicating that $\mathbf{1 2}$-a is most certainly the thermodynamic product. Similar energy differences in favour of the 1,5-regioisomer were obtained when chloroform was considered as solvent in the study, indicating that solvent polarity has no effect on the regiochemical course of this intramolecular 1,3-dipolar cycloaddition.

Since the installation of the $\mathrm{N}$-benzyltriazole moiety on the bisfuctionalized 5-azido 3$O$-propargyl xylofuranose template 11 towards the desired 5-azido isonucleoside appeared not to be feasible, an alternative pathway consisting on the introduction of an azide functionality on the 5-hydroxylated 3-O-( $\mathrm{N}$-benzyltriazolyl)methyl isonucleoside 4 was undertaken. Thus, 4 was subjected to a sequence of tosylation (tosyl chloride/pyridine) and nucleophilic displacement with sodium azide, leading to $\mathbf{1 3}$ in $78 \%$ overall yield. Staudinger-type reaction of 13 with triethyl phosphite afforded quantitatively the isonucleoside 5-phosphoramidate $\mathbf{1 4}$.

In an attempt to synthesize an isonucleoside phosphonate as a more stable analog of $\mathbf{7}$, the synthesis of a 5-bromo xylofuranose precursor to be further used in an Arbuzov reaction was carried out. Hence, bromination at C-5 of the 3-O-propargyl xylofuranose derivative 3 via Appel reaction using carbon tetrabromide and triphenylphosphine in dicloromethane at room temperature lead to the 5-bromo derivative $\mathbf{1 5}$, although in a 
modest yield (24\%), along with a mixture of degradation products. This compound was found to be unstable at room temperature and when treated with triethylphosphite, no formation of the desired phosphonate was detected. On the other hand, the bromination of the triazole isonucleoside 4 could not be accomplished either using the abovementioned Appel conditions or by treatment with phosphorous tribromide, upon which only degradation of the starting material occurred.

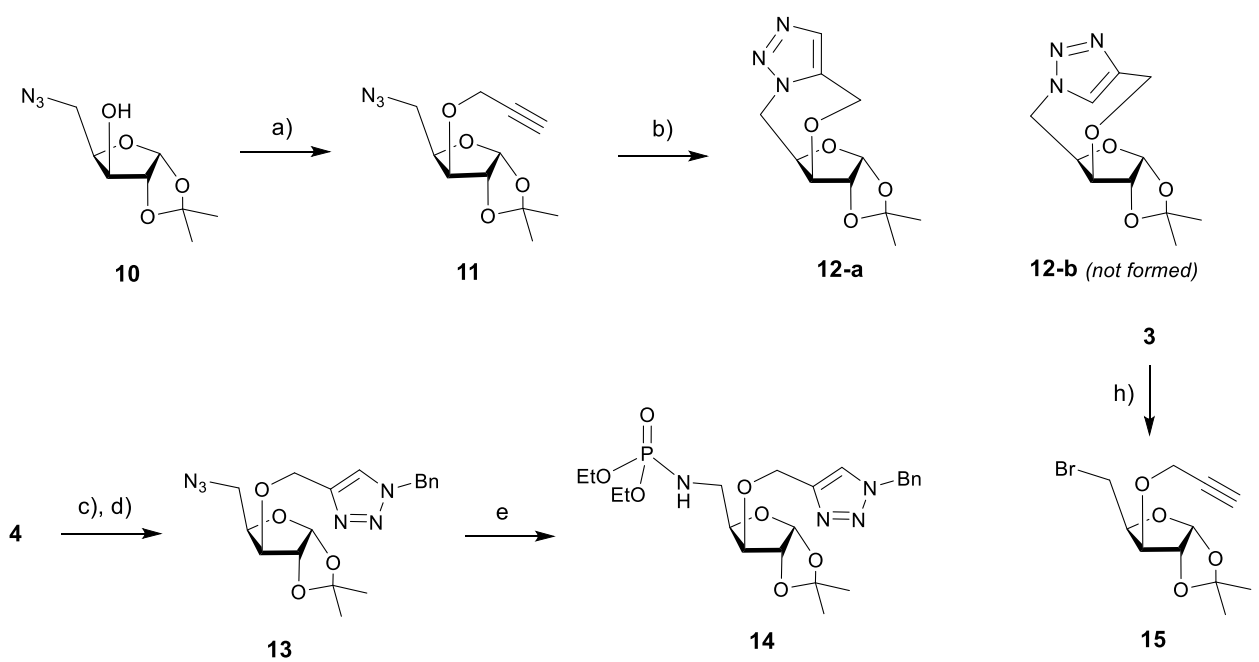

Scheme 2. Reagents and conditions: (a) $\mathrm{C}_{3} \mathrm{H}_{3} \mathrm{Br}, \mathrm{NaH}, \mathrm{DMF}$, r. t., 5 min., 95\%; (b) toluene, $100{ }^{\circ} \mathrm{C}, 2$ h, 67\%; (c) TsCl, py, r. t., 16 h; (d) $\mathrm{NaN}_{3}$, DMF, $80{ }^{\circ} \mathrm{C}, 16$ h, 78\%, 2 steps; (e) $\mathrm{P}(\mathrm{OEt})_{3}, \mathrm{CH}_{2} \mathrm{Cl}_{2}$, r. t., 48 h, 96\%; (f) $\mathrm{CBr}_{4}, \mathrm{PPh}_{3}$, DCM, r. t., 24 h, $23 \%$.

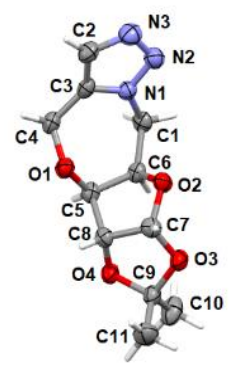

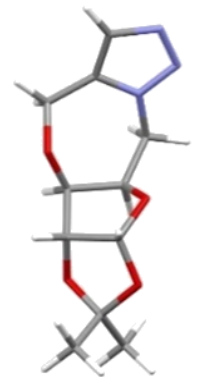

B (12-a)

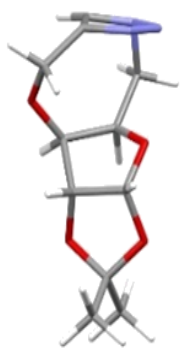

C (12-b)

1,4-disubstituted triazole

$\begin{array}{lll}\Delta H(\mathrm{kcal} / \mathrm{mol}) & 0 & 55.7 \\ \Delta G(\mathrm{kcal} / \mathrm{mol}) & 0 & 55.1\end{array}$

Fig. 2. (A) X-ray molecular structure of 12-a. (B, C) Optimized structures of 12-a and 12-b in toluene (PBE1PBE/6-311G**) and respective calculated enthalpy and Gibbs free energy. The values for the lowest energy regioisomer (12-a, structure B) were set as reference. 
5-Azido xyfuranoses were the precursors used for the synthesis of a variety of xylofuranos-5'-yl triazole isonucleosides B (Fig. 1) and their derivatives comprising additional phosphate, phosphoramidate or phosphonate groups as potential mimetics of sugar diphosphates (D, Fig. 1). Thus, the 5-azido 3-O-benzyl and 3-O-dodecyl xylofuranose derivatives $(\mathbf{1 8}, \mathbf{1 9})$ were synthesized by tosylation of the partially protected xylofuranoses $\mathbf{1 6}^{13 \mathrm{e}}$ and $\mathbf{1 7}^{8 \mathrm{~b}}$ and subsequent tosylate substitution with sodium azide (Scheme 3). Thermal cycloaddition between the 3-O-benzylated precursor 18 and propargyl alcohol in refluxing toluene afforded the 4-hydroxymethyl-1-xylofuranosyl triazole 20 and the 1,5-regioisomer 21 in $46 \%$ and 36\% yields, respectively, which were clearly identified based on the ${ }^{13} \mathrm{C}$ NMR chemical shifts of their triazole carbon signals. While in the 1,4-disubstituted triazole 20, the signals for C-4 and C-5 appeared at 147.5 ppm and $123.0 \mathrm{ppm}$, respectively, in the 1,5-dissubstituted counterpart 21, C-4 and C-5 resonated at $133.3 \mathrm{ppm}$ and $137.3 \mathrm{ppm}$, respectively. These characteristic ${ }^{13} \mathrm{C} \mathrm{NMR}$ chemical shift differences between 1,4 and 1,5-disubstituted triazoles were considered for the structural assignment of the different regioisomers of the further triazole derivatives described below. Compound $\mathbf{2 0}$ was obtained as colourless crystals (prisms), allowing its X-ray crystallographic analysis which confirmed the 1,4-regiochemistry at the triazole unit and revealed that the xylofuranose ring adopts a $\mathrm{C} 3$-endo conformation (Fig. 3a). The crystal packing of this compound revealed that the molecules forms $C_{1}^{1}(5)$ infinite linear chains that are sustained by a $\mathrm{OH} \cdots \mathrm{N} 1$ hydrogen bond $\left(d_{\mathrm{OH} \cdots \mathrm{N} 1}=\right.$ 2.02(5) $\AA$ ) (Fig. 3b). This motif is reinforced by a non-classical H-bond C3H...N2 $\left(d_{\mathrm{C} 3 \mathrm{H} \cdots \mathrm{N} 2}=2.59(3) \AA\right)$. The overall packing is completed by other four non classical $\mathrm{H}$ bonds of the type $\mathrm{CH} \cdots \mathrm{O}$ (two intermolecular bonds), $\mathrm{CH} \cdots \mathrm{N}$ and $\mathrm{CH} \cdots \pi\left(d_{\mathrm{C} 1 \mathrm{H} \cdots \mathrm{O} 4}=\right.$ $2.47(4) \AA, d_{\mathrm{C} 7 \mathrm{H} \cdots \mathrm{O} 1}=2.49(3) \AA, d_{\mathrm{C} 16 \mathrm{H} \cdots \mathrm{N} 2}=2.62(3) \AA, d_{\mathrm{C} 12 \mathrm{H} \cdots \pi}=3.3(1) \AA$, respectively). A similar cycloaddition procedure was applied to the 3-O-dodecyl counterpart 19, leading again to the 1,4-disubstituted triazole 22 in a higher yield (54\%) than that obtained for the 1,5-dissubstituted congener 23 (31\%). The hydroxymethyltriazole $5^{\prime}-$ isonucleosides 20-23 were then subjected to phosphorylation with diethyl phosphorochloridate under basic conditions as already mentioned for $\mathbf{3}$. The corresponding [(xylofuranos-5-yl)triazolyl]methyl phosphates 24-27 were obtained in good to excellent yields (68-93\%). It is noteworthy to mention that in the case of the ${ }^{1} \mathrm{H}$ NMR spectra of the 4-phosphonoxymethyl-1-xylofuranosyl triazoles $\mathbf{2 4}$ and $\mathbf{2 6}$, the signal corresponding to the protons of the oxymethylene group linked to the triazole unit appeared as a doublet at $c a .5 .2 \mathrm{ppm}$ with a coupling constant of $J_{\mathrm{CH}, \mathrm{P}}=8.8 \mathrm{~Hz}$, while in the case of the 1,5-disubstituted regioisomers 25 and 27, these protons resonated as doublet of doublets each $(\delta=c a .5 .3$ and $5.2 \mathrm{ppm})$ from an ABX system resulting from the geminal coupling $\left({ }^{2} J_{\mathrm{a}, \mathrm{b}}=\mathrm{ca} .13 \mathrm{~Hz}\right)$ and the individual couplings with the phosphorous atom $\left(J_{\mathrm{H}-\mathrm{a}, \mathrm{P}}=9.2 \mathrm{~Hz}\right.$ and $\left.J_{\mathrm{H}^{-} \mathrm{b}, \mathrm{P}}=8.4 \mathrm{~Hz}\right)$. Moreover, the ${ }^{13} \mathrm{C} \mathrm{NMR}$ signals for the triazole quaternary carbon atom atoms (C-4 or C-5) of the (triazolyl)methyl phosphates 24-27 appeared as doublets due to coupling with the phosphorous atom $\left(J_{\mathrm{C}, \mathrm{P}}=c a .7 .5 \mathrm{~Hz}\right)$. 


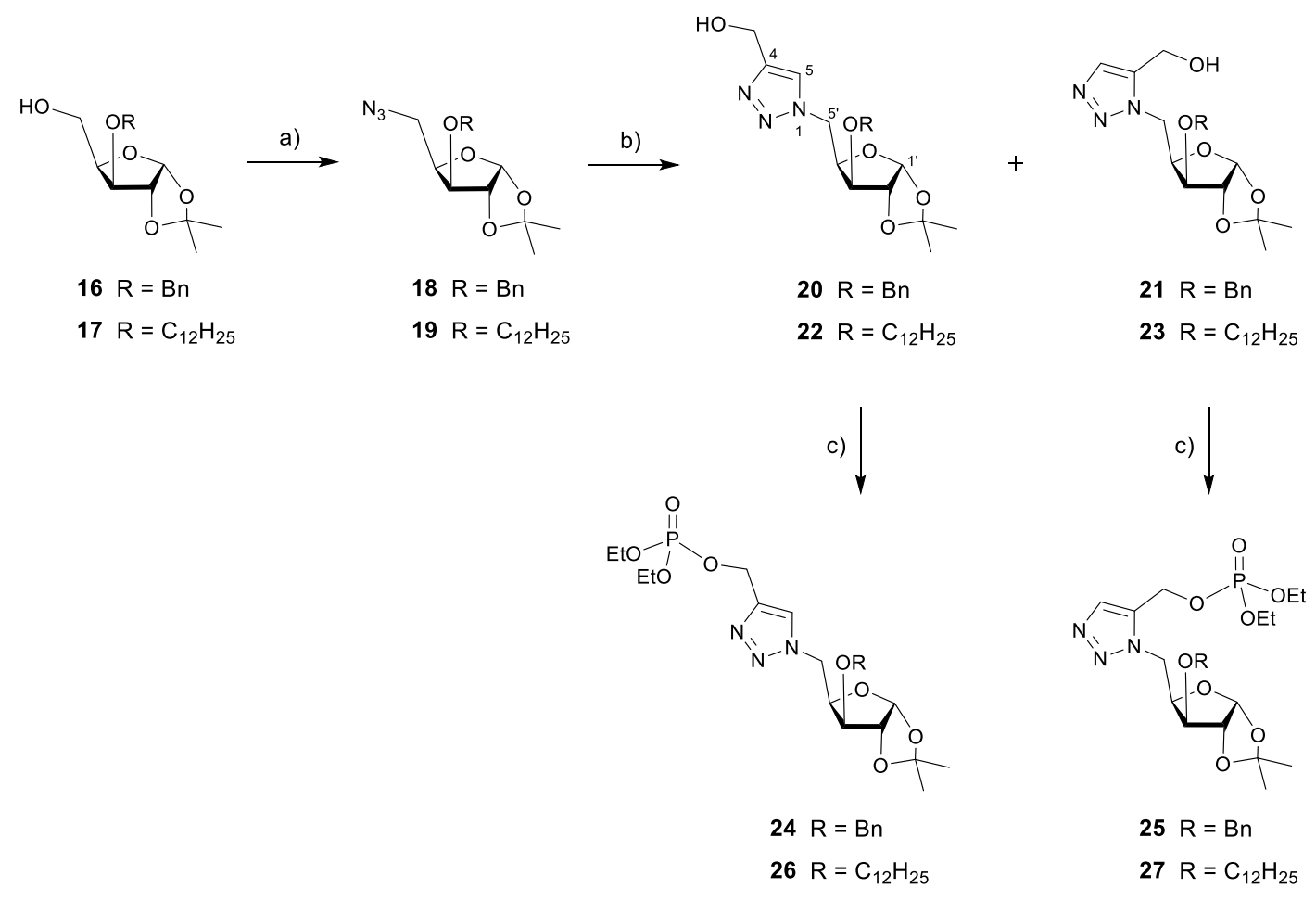

Scheme 3. Reagents and conditions: (a) TsCl, py, r. t., 16 h; (b) $\mathrm{NaN}_{3}, \mathrm{DMF}, 80{ }^{\circ} \mathrm{C}, 16$ h, 80\% (18), 77\% (19) ${ }^{8 b}, 2$ steps; (b) $\mathrm{C}_{3} \mathrm{H}_{3} \mathrm{OH}$, toluene, $110{ }^{\circ} \mathrm{C}, 24 \mathrm{~h}, 46 \%$ (20), 36\% (21), or 48 h, 54\% (22), $31 \%$ (23); (c) ClPO(EtO) $)_{2}, \mathrm{NEt}_{3}, \mathrm{DMAP} \mathrm{CH}_{2} \mathrm{Cl}_{2}$, r. t., 16 h, $78 \%$ (24), 68\% (25), 81\% (26), 93\% (27).

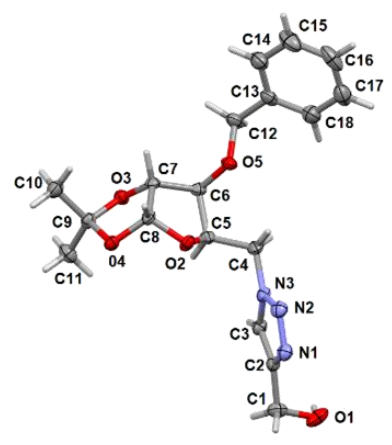

$\mathbf{a}$

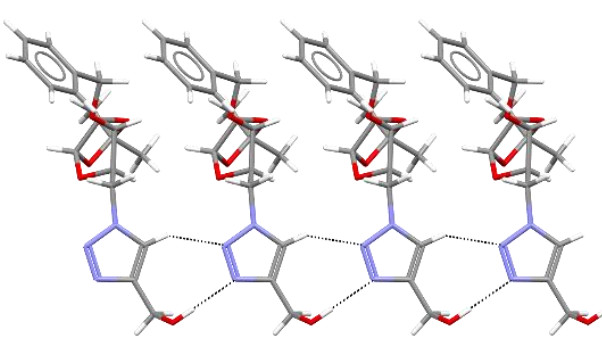

b

Fig. 3. (a) X-ray molecular structure of the 4-hydroxymethyl-1-xylofuranosyl triazole 20; (b) $C_{1}^{1}(5)$ infinite chain synthon that is formed through $\mathrm{OH} \cdots \mathrm{N} 1$ hydrogen bonds.

The 5- $O$-protected azido furanoses 18 and 19 were also subjected to thermal cycloaddition reactions with propargylamine in refluxing toluene (Scheme 4). The 5azido-3- $O$-benzyl xylofuranose derivative 18 were converted within $24 \mathrm{~h}$ into the 4 - and 5-aminomethyl-1-xylofuranosyl triazoles 28-29 in a modest total yield of $6 \%$ and in a regioisomeric ratio of 1:1. This low conversion could not be improved either with 
longer reaction times or with an increase in the amount of propargylamine, with most of 18 remaining unreacted. The 1,5-regioisomer 29 could not be isolated, being inseparably contaminated with traces of the starting material. On the other hand, when the 3-O-dodecylated derivative 19 was subjected to similar cycloaddition conditions, the 1,4- and the 1,5-disubstituted triazoles $\mathbf{3 0}$ and $\mathbf{3 1}$ were obtained in $20 \%$ yield each. Treatment of the aminomethyltriazole 5 '-isonucleosides 28, 30 and $\mathbf{3 1}$ with diethyl phosphorochloridate furnished the expected phosphoramidate derivatives 32-34 in moderate yields (50-56\%). The reaction proceeded reasonably without base. In fact the addition of trimethylamine and DMAP to the reaction medium proved to promote the formation of side products, as detected by TLC. In the ${ }^{1} \mathrm{H}$ NMR spectra of the 4phosphonoaminomethyl-1-xylofuranosyl triazoles $\mathbf{3 2}$ and 33, the aminomethylenic protons appeared as a doublet of doublet at $c a .4 .2 \mathrm{ppm}$ with coupling with the phosphorous atom $\left(J_{\mathrm{CH} 2, \mathrm{P}}=c a .11 \mathrm{~Hz}\right)$ and with the nitrogen-bound proton $\left(J_{\mathrm{CH} 2, \mathrm{NH}}=7\right.$ $\mathrm{Hz})$. On the other hand, the methylenic protons in the 1,5-disubstituted triazole $\mathbf{3 4}$ gave signals corresponding to the parts $\mathrm{A}$ and $\mathrm{B}$ of an $\mathrm{ABMX}$ system $\left(\mathrm{ABM}={ }^{1} \mathrm{H}, \mathrm{X}={ }^{31} \mathrm{P}\right)$, showing the geminal coupling $\left({ }^{2} J_{\mathrm{a}, \mathrm{b}}=15.7\right)$ in addition to the individual ${ }^{3} J$ couplings with the phosphorous atom $\left(J_{\mathrm{H}-\mathrm{a}, \mathrm{P}}=9.5, J_{\mathrm{H}-\mathrm{b}, \mathrm{P}}=12.1\right)$ and with the phosphonoamino proton $\left(J_{\mathrm{H}-\mathrm{a}, \mathrm{NH}}=9.5, J_{\mathrm{H}-\mathrm{b}, \mathrm{N} H}=5.4\right)$. Similarly to that observed in the ${ }^{13} \mathrm{C}$ NMR spectra of their phosphate counterparts 24-27, the triazole quaternary carbon atoms of the (triazolyl)methyl phosphoramidates 32-34 resonated as doublets with $J_{\mathrm{C}, \mathrm{P}}=c a .6 \mathrm{~Hz}$.

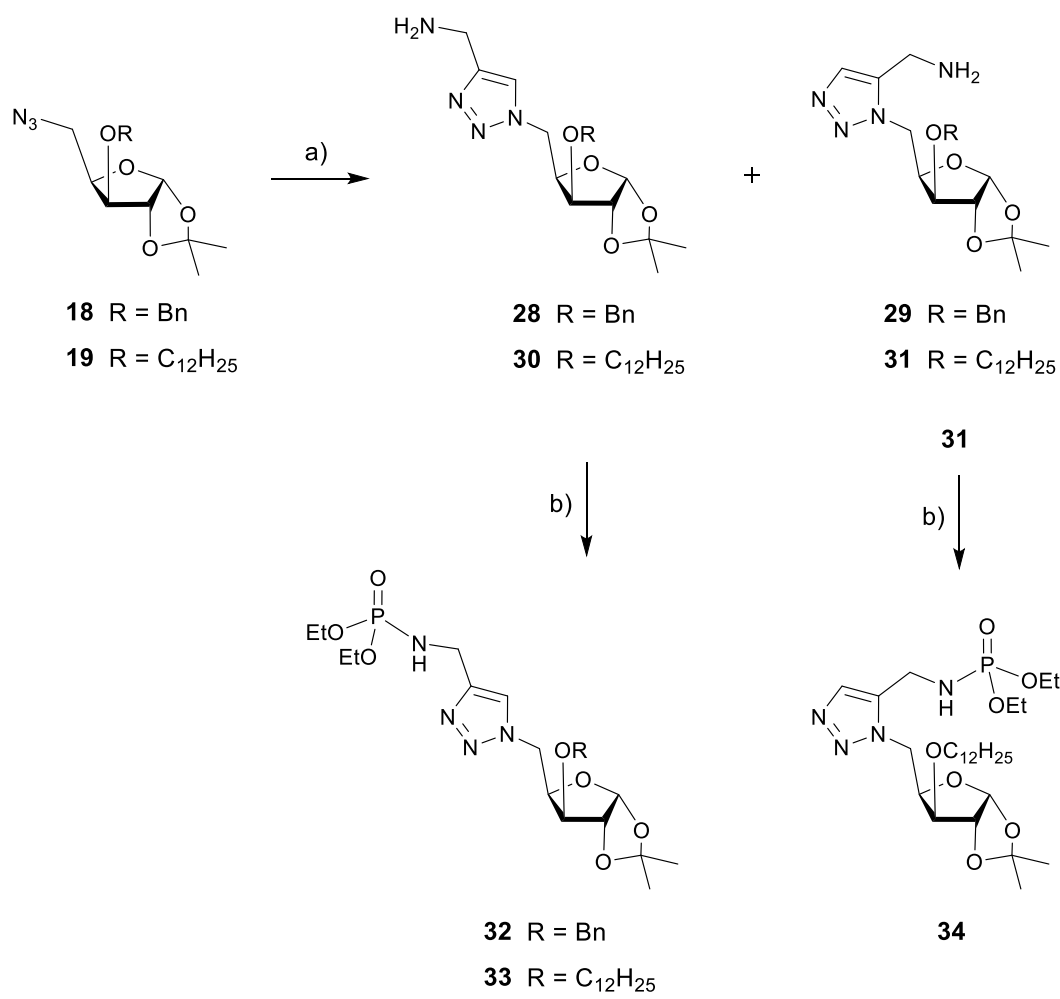

Scheme 4. Reagents and conditions: (a) propargylamine, toluene, $110{ }^{\circ} \mathrm{C}, 24 \mathrm{~h}, 3 \%$ (28), 3\% (29), or 16 h, 20\% (30), 20\% (31); (b) $\mathrm{ClPO}(\mathrm{EtO})_{2}, \mathrm{CH}_{2} \mathrm{Cl}_{2}$, r.t., 2 h, $56 \%$ (32), $50 \%(33), 52 \%(34)$. 
Towards the access to phosphonate analogs of 24-26 and 32-33, the 5-azido xylofuranoses 18 and 19 were engaged in cycloaddition with propargyl bromide under thermal conditions (Scheme 5). The reactions employing both the 3-O-benzyl and 3-Ododecyl precursors led to 4-bromomethyl-1-xylosyl-1,2,3-triazoles 35, 37 as major products in moderate yields (48-58\%), along with the 1,5-disubstituted regioisomers 36 and 37 obtained in only $2 \%$ and $5 \%$ yields, respectively. X-ray diffraction analysis of the crystals obtained for the 3'-O-benzyl bromomethyl triazole 5'-isonucleoside $\mathbf{3 5}$ proved its structure in which the xylofuranose ring is in the $\mathrm{C} 3$-endo conformation (Fig. 4a). Although in the packing of this compound the only intermolecular contacts observed are non-classical H-bonds, there are similarities between the crystalline structures of compound $\mathbf{2 0}$ and compound 35. The 3D arrangement of compound $\mathbf{3 5}$ is composed by $C_{1}^{1}(5)$ infinite non linear chains wich are supported by a non-classical $\mathrm{C} 1 \mathrm{H} \cdots \mathrm{N} 2$ interaction $\left(d_{\mathrm{C} 1 \mathrm{H} \cdots \mathrm{N} 2}=2.40 \AA\right)$. In these chains the adjacent molecules are rotated $180^{\circ}$ in order to have a more effective packing (Fig. 4b). These chains are linked to neighboring ones by non-classical hydrogen bonds of the type $\mathrm{CH} \cdots \mathrm{O}\left(d_{\mathrm{C} 6 \mathrm{H} \cdots \mathrm{O} 1}=\right.$ $2.53 \AA$ and $d_{\mathrm{C} 4 \mathrm{H} \cdots \mathrm{O} 2}=2.64 \AA$ ) and of the genre $\mathrm{CH} \cdots \pi$ between the triazole group and the phenyl group $\left(d_{\mathrm{C} 3 \mathrm{H} \cdots \pi}=3.03 \AA\right)$, fulfilling the $3 \mathrm{D}$ packing. A distinct ${ }^{13} \mathrm{C} \mathrm{NMR}$ spectral feature observed for the 1,4 and 1,5-regioisomers is the chemical shift for the bromomethylenic carbon, which appears at $c a . \delta 21.7-21.8 \mathrm{ppm}$ in the 4-bromomethyl triazole derivatives $\mathbf{3 5 / 3 7}$ and at $\delta 17.5 \mathrm{ppm}$ in the 5-bromomethyl congeners $\mathbf{3 6 / 3 8}$. The different regiochemical outcome of the 1,3-dipolar cycloaddition reactions of 18-19 with propargyl bromine relatively to that achieved when using propargyl alcohol and propargylamine, may be explained by the possibility of stabilization of the 1,5disubstituted hydroxy- $(\mathbf{2 1}, \mathbf{2 3})$ and aminomethyl $(\mathbf{2 9}, \mathbf{3 1})$ triazole derivatives through hydrogen bond interactions between the terminal hydroxyl or amino groups and the furanose endocyclic oxygen atom. In the 5-bromomethyl-1-xylosyl triazoles, not only these interactions are absent, but also lone pair repulsions between the bromine and the ring oxygen atoms, which are closer in the 1,5-regioisomers, may destabilize their formation, resulting therefore in a high regioselectivity for the 1,4-disubstituted derivatives.

The bromomethyl triazoles $\mathbf{3 5}, \mathbf{3 7}$ and $\mathbf{3 8}$ were submitted to a Michaelis-Arbuzov reaction with triethylphosphite at $110{ }^{\circ} \mathrm{C}$ to afford the [(xylofuranos-5yl)triazolyl]methyl phosphonates 39-41 in yields ranging from $75 \%$ to $96 \%$. In the ${ }^{1} \mathrm{H}$ NMR spectra of 39-41, the phosphonomethylenic protons resonate between $\delta 3.5$ to 3.2 ppm as parts $\mathrm{A}$ and $\mathrm{B}$ of an $\mathrm{ABX}$ system, with geminal couplings $\left({ }^{2} J_{\mathrm{a}, \mathrm{b}}\right)$ of $c a .21 \mathrm{~Hz}$ and individual three-bond ${ }^{31} \mathrm{P}-{ }^{1} \mathrm{H}$ couplings $\left(J_{\mathrm{H}-\mathrm{a}, \mathrm{P}}\right.$ and $\left.J_{\mathrm{H}-\mathrm{b}, \mathrm{P}}\right)$ of $c a$. $16 \mathrm{~Hz}$. Their ${ }^{13} \mathrm{C}$ NMR spectra showed both quaternary and methinic carbon atoms of the triazole units resonating as doublets with $J_{\mathrm{C}, \mathrm{P}}$ of $c a$. $7 \mathrm{~Hz}$ and 3-4 Hz, respectively. Moreover, a large $J_{\mathrm{C}, \mathrm{P}}$ coupling of $c a \cdot 143-144 \mathrm{~Hz}$ was detected for the phosphonomethylenic carbon atoms, whose signals appeared at $\delta 24.3 \mathrm{ppm}$ in the 1,4-dissubstituted phosphonomethyl triazoles 39-40 and at $21.9 \mathrm{ppm}$ in the 1,5-disubstituted derivative $\mathbf{4 1}$. 

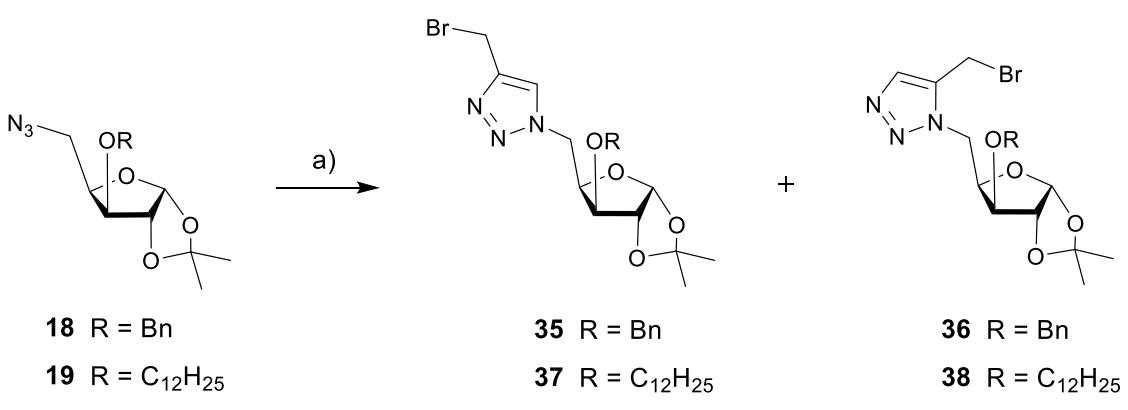

$35 \mathrm{R}=\mathrm{Bn}$
$37 \mathrm{R}=\mathrm{C}_{12} \mathrm{H}_{25}$

$36 \mathrm{R}=\mathrm{Bn}$

$38 \mathrm{R}=\mathrm{C}_{12} \mathrm{H}_{25}$

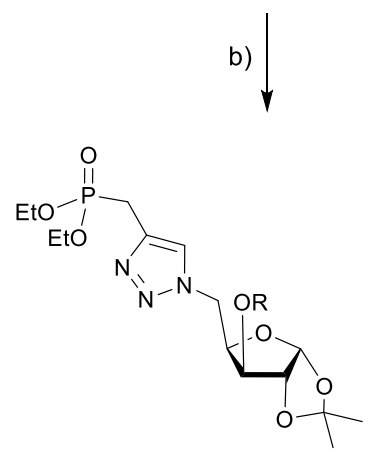

$39 \mathrm{R}=\mathrm{Bn}$

b)

$40 \mathrm{R}=\mathrm{C}_{12} \mathrm{H}_{25}$

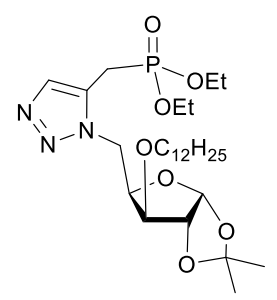

$41 \mathrm{R}=\mathrm{Bn}$

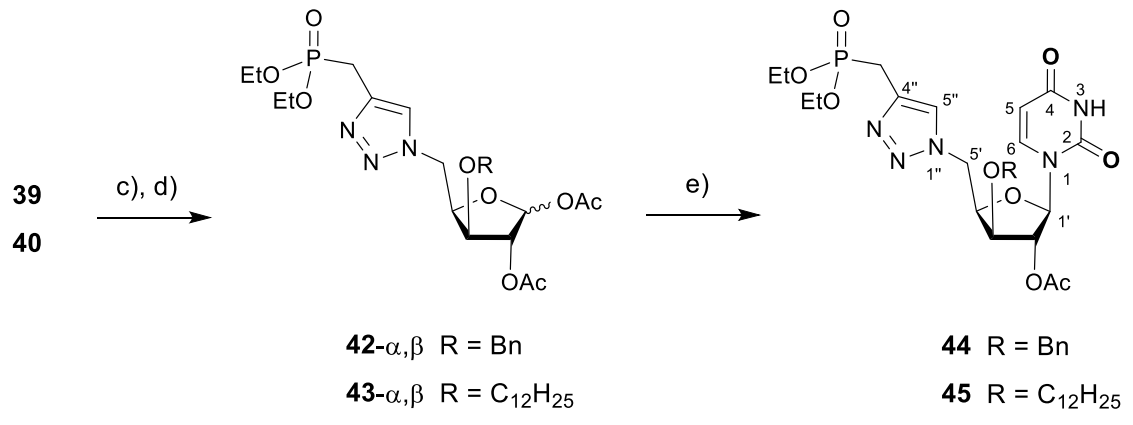

Scheme 5. Reagents and conditions: (a) $\mathrm{C}_{3} \mathrm{H}_{3} \mathrm{Br}$, toluene, $110{ }^{\circ} \mathrm{C}, 16 \mathrm{~h}, 48 \%$ (35), $2 \%$ (36), or 48 h, 58\% (37), 5\% (38); (b) $\mathrm{P}(\mathrm{EtO})_{3}, 110{ }^{\circ} \mathrm{C}, 3 \mathrm{~h}, 83 \%$ (39), $96 \%$ (40), $76 \%$ (41); (c) TFA (65\% aq. soln), r.t., 2 h (d) $\mathrm{Ac}_{2} \mathrm{O}$, py, r.t., 1.5 h, 51\% (42), 68\% (43), 2 two steps; (e) uracil, BSA, TMSOTf, $\mathrm{CH}_{3} \mathrm{CN}, 65^{\circ} \mathrm{C}$, MW, max. $150 \mathrm{~W}, 45 \mathrm{~min}, 36 \%$ (44), $16 \%$ (45).

The structural arrangement of [(furanos-5-yl)triazolyl]methyl phosphonates as potential stable mimetics of sugar diphosphates comprising a hydrolytically-resistant (triazolyl)methyl phosphonate system, motivated the insertion of a nucleobase at the anomeric position at the skeleton towards nucleotide mimetics of type $\mathbf{E}$ (Fig. 1). A 7methylguanosine-based molecule of this type of structure was previously synthesized through cycloaddition between 5-azido methylguanosine and propargylphosphonate in the context of accessing potential inhibitors of a cytosolic $5^{\prime}$-nucleotidase. ${ }^{21 \mathrm{~d}}$ Thus, compounds 39-40 were converted into the corresponding 1,2-di- $O$-acetyl xylofuranosyl 
donors 42-43 through acidic hydrolysis (65\% trifluoroacetic acid aqueous soln.) of the 1,2-acetonide group followed by acetylation $\left(\mathrm{Ac}_{2} \mathrm{O} /\right.$ pyridine). Further $\mathrm{N}$-glycosidation with uracil, which was activated by silylation with bis(trimethylsilyl)acetamide (BSA), was carried out in the presence of trimethylsilyl triflate as Lewis acid and under microwave irradiation $(150 \mathrm{~W}$, Pmax $250 \mathrm{Psi})$ in acetonitrile at $65^{\circ} \mathrm{C}$ leading to the $\mathrm{N}^{1}$ linked uracil nucleosides $\mathbf{4 4}$ and $\mathbf{4 5}$ in yields of $36 \%$ and 16\%, respectively. HMBC correlations between the anomeric proton $(\mathrm{H}-1)$ and both $\mathrm{C}-2 / \mathrm{C}-6$ of the uracil unit confirmed the regiochemistry of the nucleosidic linkage.

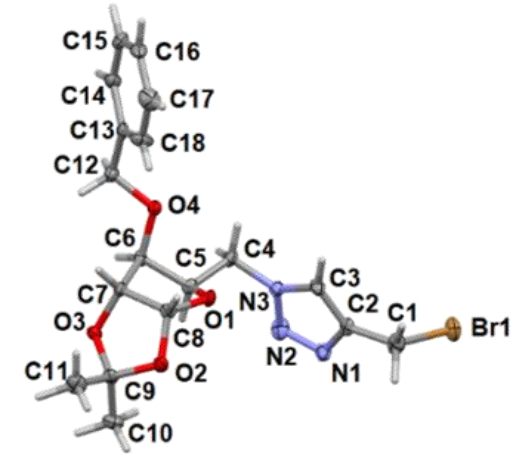

$\mathbf{a}$

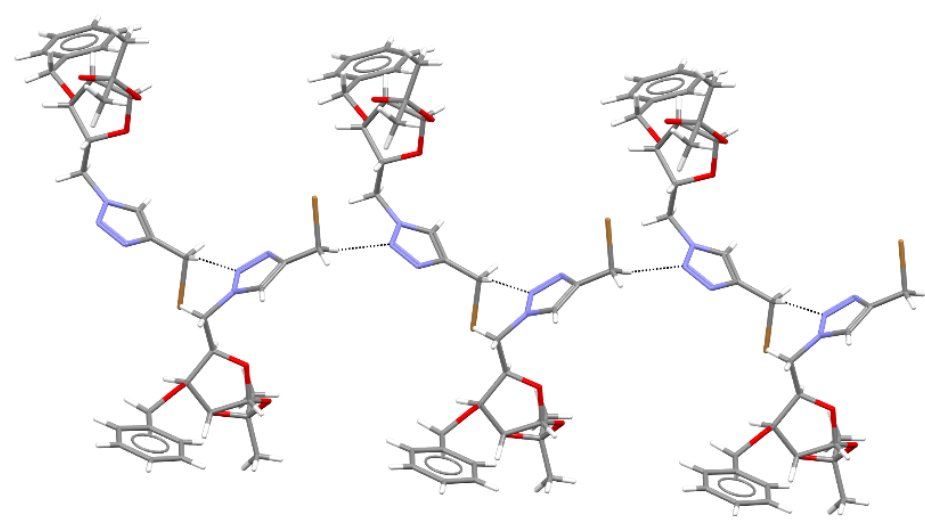

b

Fig. 4. (a) X-ray molecular structure of the bromomethyl triazole 5'-isonucleoside 35; (b) $C_{1}^{1}(5)$ infinite non-linear chains that compose the $3 \mathrm{D}$ packing of this compound.

\section{Conclusions}

This contribution showed the synthesis of a diversity of new isonucleosides, isonucleotides and nucleotide mimetics based on xylofuranosyl moieties and containing a 1,2,3-triazole unit, comprising a total of 30 molecules. 3'-O-Methyl-branched benzyltriazole isonucleosides and their isonucleotides comprising 5 '-di- $O$-ethyl/phenyl phosphate and 5'-di- $O$-ethyl phosphoramidate groups, which are previously unreported types of structural frameworks, were accessed by efficient synthetic pathways from a 1,2-diacetonide-protected 3-O-propargyl xylofuranose precursor. The CuI/Amberlyst A21-catalyzed cycloaddition reactions with benzyl azide occurred in high yields (92$98 \%$ ) for the partially protected 3-O-propargyl xylofuranose derivative and for its corresponding xylofuranos-5-yl diethyl phosphate, while the xylofuranos-5-yl diphenyl phosphate led to the corresponding triazole-containing isonucleotide in a relatively lower yield (74\%) most likely due to the hiher steric hindrance arising from the diphenyl phosphate moiety. Triazole xylofuranos-5'-yl isonucleosides containg C4/C5linked hydroxyl-, amino- or bromomethyl groups were synthesized from 5-azido 1,2-O- 
isopropylidene xylofuranoses containing 3-O-benzyl or 3-O-dodecyl groups. In general lower total yields $(6 \%-82 \%)$ were obtained for the thermal cycloaddition reactions starting from the 3-O-benzylated xylofuranose precursor than when using the 3-Ododecyl counterpart (40\%-85\%), which reflects the higher steric constrain created by the $O$-benzyl substituent. Significant lower conversions were achieved when using propargyl amine, which is in accordance with its lower electron-deficient character relatively to that of propargyl alcohol or propargy bromide, being therefore the less reactive propargyl derivative herein used in the Huisgen cycloaddition reactions. Hydrogen-bond interactions, which may stabilize 5-hydroxy- and 5-aminomethyl-1xylosyl triazoles, and lone electron pair repulsions, which eventually destabilize their bromomethyl counterparts, might explain the higher regioselectivity to 1,4-dissubstitued triazoles when using propargyl bromide.

The hydroxy-, amino- and bromomethyl 5'-triazole isonucleosides were efficiently converted into their [(xylofuranos-5'-yl)triazolyl]methyl phosphate, phosphoramidate and phosphonates, respectively, in which the structural fragment linked at the furanose unit at C-5 is a potential and relatively stable bioisostere of a disphosphate moiety. The (triazolyl)methyl phosphoramidate skeleton is herein reported for the first time. $\mathrm{N}$ Glycosylation of uracil with 1,2-di-O-acetylated 5-[4-(phosphonomethyl)triazolyl] xylofuranoses afforded the corresponding $\mathrm{N}^{1}$-linked uracil nucleosides as nucleotidelike molecules. The low yields (16\%-36\%) obtained in the nucleosidation reactions may be due to the presence of the electron-withdrawing (triazolyl)methyl phosphonate moiety in the glycosyl donors, which affects their reactivity by destabilizing the intermediate glycosyl oxocarbenium ion formed during the reaction.

In view of the biological profile of nucleoside and nucleotide analogs and the interest in the search for more cell-permeable, stable and bioactive molecules of these groups that can display new mechanisms of action or open new therapeutic oportunities, the structures of the newly synthesized compounds, which include innovative skeletons, motivate further studies to exploit their biological potential.

\section{Experimental Section}

\section{Chemistry}

\section{General methods}

Chemicals were purchased from Sigma-Aldrich and Alfa Aesar. The progress of the reactions was monitored by thin layer chromatography (TLC) using Merck $60 \mathrm{~F}_{254}$ silica gel aluminum plates with detection under UV light $(254 \mathrm{~nm})$ and/or by immersion on a solution of $10 \% \mathrm{H}_{2} \mathrm{SO}_{4}$ in EtOH or with the Hanessian stain (solution of cerium (IV) sulfate $(0.2 \% \mathrm{w} / \mathrm{v})$ and ammonium molybdate $(5 \% \mathrm{w} / \mathrm{v})$ in $\mathrm{H}_{2} \mathrm{SO}_{4}(6 \%$ aq.) followed by heating $\left(200{ }^{\circ} \mathrm{C}\right)$. Flash column chromatography was performed on silica gel $60 \mathrm{G}$ 
(0.040-0.063 mm, E. Merck). NMR spectra were acquired with a BRUKER Avance 400 spectrometer operating at $400.13 \mathrm{MHz}$ for ${ }^{1} \mathrm{H}, 100.62 \mathrm{MHz}$ for ${ }^{13} \mathrm{C}$ and at 161.91 $\mathrm{MHz}$ for ${ }^{31} \mathrm{P}$. Chemical shifts are given in parts per million and are reported relative to internal TMS, in the case of ${ }^{1} \mathrm{H} \mathrm{NMR}$ spectra in $\mathrm{CDCl}_{3}$, or relative to the respective solvent peak as reference. ${ }^{31} \mathrm{P}$ NMR Spectra were calibrated according to the IUPAC recommendations for chemical shift referencing. ${ }^{32}$ Coupling constants $(\mathrm{J})$ are reported in hertz (Hz). Signal assignments were made with aid from 2D NMR experiments (COSY, HSQC, HMBC). High-resolution mass spectrometry (HRMS) analyses were performed on a High Resolution QqTOF Impact II mass spectrometer from Bruker Daltonics equipped with an electrospray ion source (ESI). Spectra were recorded in positive mode with external calibration. Melting points were determined with a Stuart Scientific SMP 3 apparatus and are uncorrected. Optical rotations $\left(589 \mathrm{~nm}, 20{ }^{\circ} \mathrm{C}\right)$ were acquired on a Perkin-Elmer 343 polarimeter.

\section{1,2- $O$-isopropylidene-3-O-propargyl- $\alpha$-D-glucofuranose (2)}

To a solution of 1,2:5,6-di- $O$-isopropylidene- $\alpha$-D-glucofuranose $(1,3 \mathrm{~g}, 11.5 \mathrm{mmol})$ in DMF $(20 \mathrm{~mL})$, at $0^{\circ} \mathrm{C}$ and under nitrogen atmosphere, sodium hydride $(60 \% 0.55 \mathrm{~g}$, $13.8 \mathrm{mmol}$ ) was added. After stirring during $10 \mathrm{~min}$, propargyl bromide ( $80 \mathrm{wt} . \%$ soln. in toluene, $1.49 \mathrm{~mL}, 13.8 \mathrm{mmol}$ ) was added. The reaction was stirred at room temperature overnight. Then, it was diluted with diethyl ether and water was added. The phases were separated and the aqueous phase was extracted with diethyl ether $(2 \times)$. The combined organic layers were died with anhydrous $\mathrm{MgSO}_{4}$, filtered and the solvent was evaporated in vacuum. The obtained crude was dissolved in aq. acetic acid soln $(75 \%$, $30 \mathrm{~mL}$ ) and the reaction mixture was stirred at room temperature overnight. After coevaporation with toluene, the residue was purified by column chromatography (EtOAc/hexane, 1:4) to afforded the title compound (2.85 g, 96\%, 2 steps) as a yellow oil. $[\alpha]_{D}^{20}=-35\left(\mathrm{c}=1\right.$, in $\left.\mathrm{CH}_{2} \mathrm{Cl}_{2}\right)$. ${ }^{1} \mathrm{H}$ NMR (400 MHz, $\left.\mathrm{CDCl}_{3}\right): \delta 5.90(\mathrm{~d}, 1 \mathrm{H}, \mathrm{H}-1$, $\left.J_{1,2}=3.4\right), 4.60$ (d, 1H, H-2), 4.34 (br. d, $\left.1 \mathrm{H}, \mathrm{H}-1^{\prime} \mathrm{a}, J_{1^{\prime} \mathrm{a}, 1^{\prime} \mathrm{b}}=15.9\right), 4.27-4.20$ (m, $2 \mathrm{H}$, H-3, H-1'b), 4.15 (dd, 1 H, H-4, $J_{3,4}=2.8, J_{4,5}=8.2$ ), 3.97 (ddd, 1 H, H-5), 3.87 (dd, part A of AB system, $\left.1 \mathrm{H}, \mathrm{H}-6 \mathrm{a}, J_{6 \mathrm{a}, 6 \mathrm{~b}}=11.5, J_{5,6 \mathrm{a}}=2.5\right), 3.75\left(\mathrm{ddd}, 1 \mathrm{H}, \mathrm{H}-6 \mathrm{~b}, J_{5,6 \mathrm{~b}}=\right.$ 5.7), 2.64-2.42 (m, 3 H, H-3', OH-5, OH-6), 1.49 (s, 3 H, CH,$i$-Pr), 1.31 (s, 3 H, $\mathrm{CH}_{3}$, $i$-Pr). ${ }^{13} \mathrm{C}$ NMR (100 MHz, $\left.\mathrm{CDCl}_{3}\right): \delta 112.1(\mathrm{Cq}, i$-Pr), $105.3(\mathrm{C}-1), 82.2(\mathrm{C}-2), 81.6(\mathrm{C}-$ 3), 79.9 (C-4), 79.2 (C-2'), 75.7 (C-3'), 69.1 (C-5), 64.4 (C-6), 57.7 (C-1'), 26.8, 26.3 (2 $\times \mathrm{CH}_{3}, i$-Pr). HRMS: calcd for $\mathrm{C}_{12} \mathrm{H}_{18} \mathrm{O}_{6}[\mathrm{M}+\mathrm{H}]^{+}$259.1176, found 259.1181; calcd. for $\mathrm{C}_{12} \mathrm{H}_{18} \mathrm{O}_{6}[\mathrm{M}+\mathrm{Na}]^{+}$281.0996; found 281.1003.

\section{1,2-O-Isopropylidene-3- $O$-propargyl- $\alpha$-D-xylofuranose (3)}

To a solution of 1,2- $O$-isopropylidene-3- $O$-propargyl- $\alpha$-D-glucofuranose $(2,2.85 \mathrm{~g}, 11$ $\mathrm{mmol})$ in $\mathrm{THF} / \mathrm{H}_{2} \mathrm{O}(22 \mathrm{~mL}, 3: 1)$ at $0{ }^{\circ} \mathrm{C}$, sodium metaperiodate $\left(\mathrm{NaIO}_{4}, 4 \mathrm{~g}, 18.7\right.$ mmol) was added and the mixture was stirred at room temperature for $4 \mathrm{~h}$. The mixture was then diluted with EtOAc and it was filtered over celite. The phases were separated and the aqueous phase was extracted with EtOAc $(2 \times)$. The combined organic layers 
were dried with anhydrous $\mathrm{MgSO}_{4}$, filtered and concentrated under vacuum. The resulting residue was dissolved in $\mathrm{EtOH} / \mathrm{H}_{2} \mathrm{O}(22 \mathrm{~mL}, 2: 1)$ and at $0^{\circ} \mathrm{C}$, sodium borohydride $\left(\mathrm{NaBH}_{4}, 567 \mathrm{mg}, 15 \mathrm{mmol}\right)$ was added. The mixture was stirred at room temperature for $1 \mathrm{~h}$. Then, EtOAc was added. The mixture was washed with water and the aqueous phase was extracted with EtOAc $(2 \times)$. The combined organic layers were washed with brine soln. and were dried with anhydrous $\mathrm{MgSO}_{4}$. After filtration and evaporation of the solvents under vacuum, the residue was subjected to column chromatography (EtOAc/hexane, 1:4) to afford the title compound (1.44 g, 57\%, 2 steps) as a colourless oil. $[\alpha]_{D}^{20}=-62\left(\mathrm{c}=1\right.$, in $\left.\mathrm{CH}_{2} \mathrm{Cl}_{2}\right) .{ }^{1} \mathrm{H} \mathrm{NMR}\left(400 \mathrm{MHz}, \mathrm{CDCl}_{3}\right)$ : $\delta 5.84\left(\mathrm{~d}, 1 \mathrm{H}, \mathrm{H}-1, J_{1,2}=3.0\right), 4.54(\mathrm{~d}, 1 \mathrm{H}, \mathrm{H}-2), 4.27-4.15\left(\mathrm{~m}, 2 \mathrm{H}, \mathrm{H}-4, \mathrm{H}-1^{\prime} \mathrm{a}, J_{1^{\prime} \mathrm{a}, 1^{\prime} \mathrm{b}}\right.$ = 16.4), 4.16-4.04 (m, $2 \mathrm{H}, \mathrm{H}-1$ 'b, H-3), 3.83-3.69 (m, $2 \mathrm{H}, \mathrm{CH}_{2}-5, J_{5 \mathrm{a}, 5 \mathrm{~b}}=11.8, J_{4,5 \mathrm{a}}=$ 5.9, $J_{4,5 \mathrm{~b}}=5.4$ ), 2.80 (br. s, $\left.1 \mathrm{H}, \mathrm{OH}\right), 2.47$ (m, $1 \mathrm{H}, \mathrm{H}-3$ '), 1.41 (s, $3 \mathrm{H}, \mathrm{CH}_{3}, i$-Pr), 1.23 (s, $3 \mathrm{H}, \mathrm{CH}_{3}, i$-Pr). ${ }^{13} \mathrm{C} \mathrm{NMR}\left(100 \mathrm{MHz}, \mathrm{CDCl}_{3}\right): \delta 112.0(\mathrm{Cq}, i$-Pr), $105.2(\mathrm{C}-1), 82.4$ (C-2), 82.2 (C-3), 80.1 (C-4), 79.0 (C-2'), 75.6 (C-3'), 60.7 (C-5), 57.5 (C-1'), 26.9, 26.4 $\left(2 \times \mathrm{CH}_{3}, i\right.$-Pr). HRMS: calcd. for $\mathrm{C}_{11} \mathrm{H}_{18} \mathrm{O}_{5}[\mathrm{M}+\mathrm{Na}]^{+}$251.0890; found 251.0895.

General procedure for the $\mathrm{CuI} / \mathrm{Amberlyst} \mathrm{A}-21$ catalyzed cycloaddition between 3$O$-propargyl xylofuranose derivatives and benzyl azide

To a solution of 3-O-propargyl derivative $(0.88 \mathrm{mmol})$ in $\mathrm{CH}_{2} \mathrm{Cl}_{2}(5 \mathrm{~mL})$ under nitrogen atmosphere, benzyl azide $(0.14 \mathrm{~mL}, 1.06 \mathrm{mmol})$ and CuI/Amberlyst A-21 (0.5 mmol.g ${ }^{-}$ 1, $0.1 \mathrm{mmol} \mathrm{CuI}, 200 \mathrm{mg}$ ) were added. The mixture was stirred at room temperature for $24 \mathrm{~h}$. The catalyst was filtered off, the solvent was evaporated and the crude residue was subjected to column chromatography.

\section{3-O-(1-Benzyl-1H-1,2,3-triazol-4-yl)methyl-1,2- $O$-isopropylidene- $\alpha$-D-xylofuranose}

(4)

Obtained according to the general $\mathrm{CuI} / \mathrm{A} 21$ cycloaddition procedure, starting from 1,2$O$-isopropylidene-3- $O$-propargyl- $\alpha$-D-xylofuranose (3, $200 \mathrm{mg}, 0.88 \mathrm{mmol}$ ), benzyl azide $(0.14 \mathrm{~mL}, 1.06 \mathrm{mmol})$ and in the presence of $\mathrm{CuI} / \mathrm{A}-21$ catalyst $(0.1 \mathrm{mmol} \mathrm{CuI}$, $200 \mathrm{mg}$ ). Purification by column chromatography (EtOA/petroleum ether, 1:1) afforded the title compound $(310 \mathrm{mg}, 98 \%)$ as a colourless oil. $[\alpha]_{D}^{20}=-33\left(\mathrm{c}=1\right.$, in $\left.\mathrm{CH}_{2} \mathrm{Cl}_{2}\right)$. ${ }^{1} \mathrm{H}$ NMR (400 MHz, $\mathrm{CDCl}_{3}$ ): $\delta 7.48(\mathrm{~s}, 1 \mathrm{H}, \mathrm{H}-3$ '), 7.42-7.33 (m, $3 \mathrm{H}, \mathrm{Ph}$ ), 7.31-7.23 $(\mathrm{m}, 2 \mathrm{H}, \mathrm{Ph}), 5.92\left(\mathrm{~d}, 1 \mathrm{H}, \mathrm{H}-1, J_{1,2}=3.7\right), 5.52\left(\mathrm{~s}, 2 \mathrm{H}, \mathrm{CH}_{2} \mathrm{Ph}\right), 4.81(\mathrm{~d}, 1 \mathrm{H}$, part A of AB system, H-1'a, $\left.J_{1^{\prime} \mathrm{a}, 1^{\prime} \mathrm{b}}=12.9\right), 4.67-4.59$ (m, 2 H, H-1'b, H-2), 4.32 (ddd, 1 H, H-4), $4.08\left(\mathrm{~d}, 1 \mathrm{H}, \mathrm{H}-3, J_{3,4}=3.3\right), 3.91-3.77\left(\mathrm{~m}, 2 \mathrm{H}, \mathrm{CH}_{2}-5, J_{5 \mathrm{a}, 5 \mathrm{~b}}=11.7, J_{4,5 \mathrm{a}}=7.0, J_{4,5 \mathrm{~b}}=\right.$ 5.5), 1.46 (s, $3 \mathrm{H}, \mathrm{CH}_{3}, i$-Pr), 1.29 (s, $3 \mathrm{H}, \mathrm{CH}_{3}, i$-Pr) ppm. ${ }^{13} \mathrm{C} \mathrm{NMR}(100 \mathrm{MHz}$, $\left.\mathrm{CDCl}_{3}\right): \delta 144.6\left(\mathrm{C}-2^{\prime}\right), 134.3(\mathrm{Cq}, \mathrm{Ph}), 129.2,128.9,128.1(\mathrm{CH}, \mathrm{Ph}), 122.3\left(\mathrm{C}-3^{\prime}\right)$, 111.7 (Cq, $i$-Pr), 105.1 (C-1), 82.4 (C-2), 82.3 (C-3), 80.1 (C-4), 63.1 (C-1'), 59.5 (C-5), $54.4\left(\mathrm{CH}_{2} \mathrm{Ph}\right), 26.8,26.3\left(2 \times \mathrm{CH}_{3}, i\right.$-Pr $)$. HRMS: calcd. for $\mathrm{C}_{18} \mathrm{H}_{23} \mathrm{~N}_{3} \mathrm{O}_{5}[\mathrm{M}+\mathrm{H}]^{+}$ 362.1710, found 362.1713; calcd. for $\mathrm{C}_{18} \mathrm{H}_{23} \mathrm{~N}_{3} \mathrm{O}_{5}[\mathrm{M}+\mathrm{Na}]^{+} 384.1530$; found 384.1532 . 


\section{3-O-(1-Benzyl-1H-1,2,3-triazol-4-yl)methyl-5-O-dodecyl-1,2- $O$-isopropylidene- $\alpha$-D- xylofuranose (5)}

To a solution of 3-O-(1-benzyl-1H-1,2,3-triazol-4-yl)methyl-1,2- $O$-isopropylidene- $\alpha$-Dxylofuranose $(4,30 \mathrm{mg}, 0.08 \mathrm{mmol})$ in DMF $(2 \mathrm{~mL})$, at $0^{\circ} \mathrm{C}$ and under nitrogen atmosphere, sodium hydride $(60 \%, 4 \mathrm{mg}, 0.1 \mathrm{mmol})$ was added. After stirring during 10 min, dodecyl bromide $(0.02 \mathrm{~mL}, 0.12 \mathrm{mmol})$ was added. The reaction was stirred at room temperature overnight. Then, it was diluted with diethyl ether and water was added. The phases were separated and the aqueous phase was extracted with diethyl ether $(2 \times)$. The combined organic layers were died with anhydrous $\mathrm{MgSO}_{4}$, filtered and the solvent was evaporated in vacuum. The residue was purified by column chromatography (EtOAc/hexane, 1:3) to afford the title compound (42 $\mathrm{mg}, 95 \%$ ) as a white solid. $[\alpha]_{D}^{20}=-15\left(\mathrm{c}=1\right.$, in $\left.\mathrm{CH}_{2} \mathrm{Cl}_{2}\right)$. m.p.: $42.7-43.4{ }^{\circ} \mathrm{C} .{ }^{1} \mathrm{H}$ NMR $(400 \mathrm{MHz}$, $\mathrm{CDCl}_{3}$ ): $\delta 7.44\left(\mathrm{~s}, 1 \mathrm{H}, \mathrm{H}-3^{\prime}\right), 7.42-7.32(\mathrm{~m}, 3 \mathrm{H}, \mathrm{Ph}), 7.30-7.22(\mathrm{~m}, 2 \mathrm{H}, \mathrm{Ph}), 5.87$ (d, 1 $\left.\mathrm{H} \mathrm{H}-1, J_{1,2}=3.7\right), 5.51\left(\mathrm{~s}, 2 \mathrm{H}, \mathrm{CH}_{2} \mathrm{Ph}\right), 4.75$ (d, part A of AB system, $1 \mathrm{H}, \mathrm{H}-1^{\prime} \mathrm{a}, J_{1^{\prime} \mathrm{a}, 1^{\prime} \mathrm{b}}$ = 12.4), 4.65 (d, of AB system, $1 \mathrm{H}, \mathrm{H}-1$ 'b), 4.60 (d, $1 \mathrm{H}, \mathrm{H}-2), 4.33$ (td, $1 \mathrm{H}, \mathrm{H}-4), 3.99$ $\left(\mathrm{d}, 1 \mathrm{H}, \mathrm{H}-3, J_{3,4}=3.0\right), 3.65-3.54\left(\mathrm{~m}, 2 \mathrm{H}, \mathrm{CH}_{2}-5, J_{5 \mathrm{a}, 5 \mathrm{~b}}=10.1, J_{4,5 \mathrm{a}}=6.2, J_{4,5 \mathrm{~b}}=6.0\right)$, 3.48-3.32 (m, $2 \mathrm{H} \mathrm{CH}_{2}-1^{\prime \prime}$ ), 1.57-1.44 (m, $\left.5 \mathrm{H}, \mathrm{CH}_{2}-2^{\prime \prime}, \mathrm{CH}_{3}, i-\mathrm{Pr}\right), 1.34-1.17$ (m, $21 \mathrm{H}$, $\mathrm{CH}_{3}, i$-Pr, $\mathrm{CH}_{2}-3^{\prime \prime}$ to $\left.\mathrm{CH}_{2}-11^{\prime \prime}\right), 0.87$ (t, $\left.3 \mathrm{H}, \mathrm{CH}_{3}-12^{\prime \prime}, J=6.8\right) .{ }^{13} \mathrm{C} \mathrm{NMR}(100 \mathrm{MHz}$, $\left.\mathrm{CDCl}_{3}\right): \delta 145.3\left(\mathrm{C}-2^{\prime}\right), 134.6(\mathrm{Cq}, \mathrm{Ph}) 129.3,128.9,128.2(\mathrm{CH}, \mathrm{Ph}), 122.4\left(\mathrm{C}-3^{\prime}\right)$, 111.8 (Cq, $i$-Pr), 105.1 (C-1), 82.5 (C-2), 82.2 (C-3), 79.0 (C-4), 71.9 (C-1"), 67.8 (C5), $64.0\left(\mathrm{C}-1^{\prime}\right), 54.3\left(\mathrm{CH}_{2} \mathrm{Ph}\right), 32.0,29.8,29.8,29.7,29.6,29.5$ (C-2" to C-9") 26.9, $26.4\left(2 \times \mathrm{CH}_{3}, i\right.$-Pr), $26.2\left(\mathrm{C}-10^{\prime \prime}\right), 22.8\left(\mathrm{C}-11^{\prime \prime}\right), 14.3$ (C-12"). HRMS: calcd for $\mathrm{C}_{30} \mathrm{H}_{47} \mathrm{~N}_{3} \mathrm{O}_{5}[M+\mathrm{H}]^{+}$530.3588, found 530.3594; calcd for $\mathrm{C}_{30} \mathrm{H}_{47} \mathrm{~N}_{3} \mathrm{O}_{5}[M+\mathrm{Na}]^{+}$ 552.3408, found 552.3421.

\section{5- $O$-Diethylphosphono-1,2- $O$-isopropylidene-3- $O$-propargyl- $\alpha$-D-xylofuranose (6)}

To a solution of 1,2- $O$-isopropylidene-3- $O$-propargyl- $\alpha$-D-xylofuranose $(6,100 \mathrm{mg}$, $0.44 \mathrm{mmol})$ in dichloromethane $(5 \mathrm{~mL})$ under nitrogen atmosphere, triethylamine $(0.09$ $\mathrm{mL}, 0.66 \mathrm{mmol}$ ) was added. The mixture was cooled at $0{ }^{\circ} \mathrm{C}$ and diethyl phosphorochloridate $(0.08 \mathrm{~mL}, 0.53 \mathrm{mmol})$ and DMAP (catalytic amount, a spatula tip) were added. The solution was stirred at room temperature for $16 \mathrm{~h}$. Then, water was added to the solution, the phases were separated and the aqueous phase was extracted with $\mathrm{CH}_{2} \mathrm{Cl}_{2}$. The combined organic layers were dried with anhydrous $\mathrm{MgSO}_{4}$, filtered and the solvent was evaporated under vacuum. Purification by column chromatography (EtOAc/hexane, 1:3) afforded the title compound (157 mg, $98 \%$ ) as a colourless oil. $[\alpha]_{D}^{20}=-24\left(\mathrm{c}=1\right.$, in $\left.\mathrm{CH}_{2} \mathrm{Cl}_{2}\right) .{ }^{1} \mathrm{H} \mathrm{NMR}\left(400 \mathrm{MHz}, \mathrm{CDCl}_{3}\right): \delta 5.86\left(\mathrm{~d}, 1 \mathrm{H}, \mathrm{H}-1, J_{1,2}=\right.$ 3.7), 4.58 (d, 1 H, H-2), 4.39 (ddd, 1 H, H-4), 4.24-4.00 (m, 9 H, H-3, H-5a, H-5b, $2 \times$ $\mathrm{CH}_{2}$, Et, $\left.\mathrm{CH}_{2}-1^{\prime}\right), 2.47$ (t, $\left.1 \mathrm{H}, \mathrm{H}-3^{\prime}, J_{1^{\prime} \mathrm{a}, 3^{\prime}}=J_{1^{\prime} \mathrm{b}, 3^{\prime}}=2.3\right), 1.44$ (s, $\left.3 \mathrm{H}, \mathrm{CH}_{3}, i-\mathrm{Pr}\right), 1.32-$ $1.23\left(\mathrm{~m}, 9 \mathrm{H}, 2 \times \mathrm{CH}_{3}, \mathrm{Et}, \mathrm{CH}_{3}, i\right.$-Pr). ${ }^{13} \mathrm{C} \mathrm{NMR}\left(100 \mathrm{MHz}, \mathrm{CDCl}_{3}\right): \delta 112.0(\mathrm{Cq}, i$-Pr), $105.2(\mathrm{C}-1), 82.0(\mathrm{C}-2), 81.1(\mathrm{C}-3), 78.8\left(\mathrm{C}-2^{\prime}\right), 78.7\left(\mathrm{~d}, \mathrm{C}-4, J_{\mathrm{C}-4, \mathrm{P}}=8.5\right), 75.5\left(\mathrm{C}-3^{\prime}\right)$, $64.8\left(\mathrm{~d}, \mathrm{C}-5, J_{\mathrm{C}-\mathrm{P}}=5.3\right), 63.99\left(\mathrm{~d}, C \mathrm{H}_{2}, \mathrm{Et}, J_{\mathrm{C}-\mathrm{P}}=5.9\right), 63.97\left(\mathrm{~d}, C_{2}, \mathrm{Et}, J_{\mathrm{C}-\mathrm{P}}=5.9\right)$, 
$57.5\left(\mathrm{C}-1^{\prime}\right), 26.8,26.3\left(2 \times \mathrm{CH}_{3}, i\right.$-Pr $), 16.1\left(\mathrm{~d}, 2 \times \mathrm{CH}_{3}, 2 \times \mathrm{Et}, J_{\mathrm{C}, \mathrm{P}}=6.9\right) .{ }^{31} \mathrm{P}-\mathrm{RMN}$ $\left(162 \mathrm{MHz}, \mathrm{CDCl}_{3}\right): \delta-1.28$. HRMS: calcd. for $\mathrm{C}_{15} \mathrm{H}_{25} \mathrm{O}_{8} \mathrm{P}[\mathrm{M}+\mathrm{H}]^{+} 387.1179$, found 387.1179; calcd. for $\mathrm{C}_{15} \mathrm{H}_{25} \mathrm{O}_{8} \mathrm{P}[\mathrm{M}+\mathrm{Na}]^{+}$365.1360, found 365.1358.

\section{3-O-(1-Benzyl-1H-1,2,3-triazol-4-yl)methyl-5-O-diethylphosphono-1,2-O- isopropylidene- $\alpha$-D-xylofuranose $(7)$}

Obtained according to the general $\mathrm{CuI} / \mathrm{A} 21$ cycloaddition procedure, starting from $5-\mathrm{O}$ diethylphosphono-1,2- $O$-isopropylidene-3- $O$-propargyl- $\alpha$-D-xylofuranose $(6,100 \mathrm{mg}$, $0.27 \mathrm{mmol})$, benzyl azide $(0.06 \mathrm{~mL}, 0.44 \mathrm{mmol})$ in the presence of CuI/A-21 catalyst (0.05 mmol CuI, $100 \mathrm{mg}$ ). Purification by column chromatography (EtOAc/petroleum ether, 1:2) afforded the title compound $(125 \mathrm{mg}, 92 \%)$ as pale yellow oil. $[\alpha]_{D}^{20}=-23$ (c = 1, in $\mathrm{CH}_{2} \mathrm{Cl}_{2}$ ). ${ }^{1} \mathrm{H}$ NMR (400 MHz, $\left.\mathrm{CDCl}_{3}\right): \delta 7.58$ (s, $\left.1 \mathrm{H}, \mathrm{H}-3^{\prime}\right), 7.41-7.25$ (m, 5 $\mathrm{H}, \mathrm{Ph}), 5.89\left(\mathrm{~d}, 1 \mathrm{H}, \mathrm{H}-1, J_{1,2}=3.7\right), 5.55,5.50\left(2 \mathrm{~d}, \mathrm{AB}\right.$ system, $2 \mathrm{H}, \mathrm{CH}_{2} \mathrm{Ph}, J_{\mathrm{a}, \mathrm{b}}=$ 15.0), 4.77 (d, $1 \mathrm{H}$, part A of AB system, H-1'a, $\left.J_{1^{\prime} \mathrm{a}, 1^{\prime} \mathrm{b}}=12.2\right), 4.66-4.59$ (m, $2 \mathrm{H}, \mathrm{H}-1^{\prime} \mathrm{b}$, H-2), 4.40 (ddd, 1 H, H-4), 4.25-4.00 (m, H-3, $\mathrm{CH}_{2}-5,2 \times \mathrm{CH}_{2}, \mathrm{Et}$ ), 1.48 (s, $3 \mathrm{H}, \mathrm{CH}_{3}, i-$ Pr), 1.34-1.26 (m, $9 \mathrm{H}, 2 \times \mathrm{CH}_{3}, \mathrm{Et}, \mathrm{CH}_{3}, i$-Pr). ${ }^{13} \mathrm{C} \mathrm{NMR}\left(100 \mathrm{MHz}, \mathrm{CDCl}_{3}\right): \delta 144.7$ $\left(\mathrm{C}-2^{\prime}\right), 134.7(\mathrm{Cq}, \mathrm{Ph}), 129.2,128.8,128.2(\mathrm{CH}, \mathrm{Ph}), 122.9\left(\mathrm{C}-3^{\prime}\right), 112.0(\mathrm{Cq}, i-\mathrm{Pr})$, $105.2(\mathrm{C}-1), 82.1(\mathrm{C}-2), 81.6(\mathrm{C}-3), 78.6\left(\mathrm{~d}, \mathrm{C}-4, J_{\mathrm{C}-4, \mathrm{P}}=8.8\right), 64.4\left(\mathrm{~d}, \mathrm{C}-5, J_{\mathrm{C}-\mathrm{P}}=5.3\right)$, $64.09\left(\mathrm{~d}, \mathrm{CH}_{2}, \mathrm{Et}, J_{\mathrm{C}-\mathrm{P}}=5.9\right), 64.05\left(\mathrm{~d}, \mathrm{CH}_{2}, \mathrm{Et}, J_{\mathrm{C}-\mathrm{P}}=5.7\right), 63.8\left(\mathrm{C}-1^{\prime}\right), 54.3\left(\mathrm{CH}_{2} \mathrm{Ph}\right)$, 26.9, $26.3\left(2 \times \mathrm{CH}_{3}, i\right.$-Pr), $16.2\left(\mathrm{~d}, 2 \times \mathrm{CH}_{3}, 2 \times \mathrm{Et}, J_{\mathrm{C}, \mathrm{P}}=6.8\right) .{ }^{31} \mathrm{P} \mathrm{NMR}(162 \mathrm{MHz}$, $\left.\mathrm{CDCl}_{3}\right): \delta-1.26$ ppm. HRMS: calcd for $\mathrm{C}_{22} \mathrm{H}_{32} \mathrm{~N}_{3} \mathrm{O}_{8} \mathrm{P}[\mathrm{M}+\mathrm{H}]^{+} 498.2000$, found 498.2009.

\section{1,2-O-Isopropylidene-5- $O$-diphenylphosphono-3- $O$-propargyl- $\alpha$-D-xylofuranose (8)}

To a solution of 1,2- $O$-isopropylidene-3- $O$-propargyl- $\alpha$-D-xylofuranose $(3,100 \mathrm{mg}$, $0.44 \mathrm{mmol})$ in toluene $(6 \mathrm{~mL})$ under nitrogen, at $0{ }^{\circ} \mathrm{C}$, diphenylphosphoryl azide $(0.11$ $\mathrm{mL}, 0.53 \mathrm{mmol})$ and $\mathrm{DBU}(0.08 \mathrm{~mL}, 0.53 \mathrm{mmol})$ were added and the mixture was stirred at room temperature for $24 \mathrm{~h}$. Additional DBU $(0.05 \mathrm{~mL}, 0.8$ equiv. $)$ was then added at $0{ }^{\circ} \mathrm{C}$ and stirring was continued at $40{ }^{\circ} \mathrm{C}$ for $16 \mathrm{~h}$. The mixture was then washed twice with $2 \mathrm{M} \mathrm{HCl}$ soln. and water. The organic phase was dried with anhydrous magnesium sulfate, filtered and the solvent was evaporated under vacuum. The crude residue was purified by column chromatography (EtOAc/hexane, 1:4) to afford the title compound $(138 \mathrm{mg}, 68 \%)$ as a colourless oil. $[\alpha]_{D}^{20}=-46(\mathrm{c}=1$, in $\mathrm{CH}_{2} \mathrm{Cl}_{2}$ ). ${ }^{1} \mathrm{H}$ NMR $\left(400 \mathrm{MHz}, \mathrm{CDCl}_{3}\right): \delta 7.42-7.15(\mathrm{~m}, 10 \mathrm{H}, 2 \times \mathrm{Ph}), 5.94\left(\mathrm{~d}, 1 \mathrm{H}, J_{1,2}\right.$ = 3.7), 4.65 (d, $1 \mathrm{H}, \mathrm{H}-2)$, 4.54-4.40 (m, $3 \mathrm{H}, \mathrm{H}-4, \mathrm{H}-5 \mathrm{a}, \mathrm{H}-5 \mathrm{~b}), 4.19-4.09$ (m, $3 \mathrm{H}, \mathrm{CH}_{2^{-}}$ $\left.1^{\prime}, \mathrm{H}-3\right), 2.47$ (t, $\left.1 \mathrm{H}, \mathrm{H}-3^{\prime}, J_{1^{\prime} \mathrm{a}, 3^{\prime}}=J_{1^{\prime} \mathrm{b}, 3^{\prime}}=2.2\right), 1.49$ (s, $3 \mathrm{H}, \mathrm{CH}_{3}, i$-Pr), 1.33 (s, $3 \mathrm{H}$, $\mathrm{CH}_{3}, i$-Pr). ${ }^{13} \mathrm{C}$ NMR $\left(100 \mathrm{MHz}, \mathrm{CDCl}_{3}\right): \delta 150.52$ (d, Cq, Ph, $\left.J_{\mathrm{C}, \mathrm{P}}=7.3\right), 150.49$ (d, Cq, $\left.\mathrm{Ph}, J_{\mathrm{C}, \mathrm{P}}=7.1\right), 129.8,125.4,120.2,120.2(\mathrm{CH}, 2 \times \mathrm{Ph}) 112.2(\mathrm{Cq}, i-\mathrm{Pr}), 105.3(\mathrm{C}-1)$, 82.1 (C-2), $81.1(\mathrm{C}-3), 78.8\left(\mathrm{C}-2^{\prime}\right), 78.5\left(\mathrm{~d}, \mathrm{C}-4, J_{\mathrm{C}-4, \mathrm{P}}=8.6\right), 75.6\left(\mathrm{C}-3^{\prime}\right), 66.2$ (d, C-5, $\left.J_{\mathrm{C}-5, \mathrm{P}}=6.2\right), 57.6\left(\mathrm{C}-1^{\prime}\right), 26.9,26.4\left(2 \times \mathrm{CH}_{3}, i\right.$-Pr $) .{ }^{31} \mathrm{P} \mathrm{NMR}\left(162 \mathrm{MHz}, \mathrm{CDCl}_{3}\right): \delta$ 
12.06. HRMS: calcd. for $\mathrm{C}_{23} \mathrm{H}_{25} \mathrm{O}_{8} \mathrm{P}[\mathrm{M}+\mathrm{H}]^{+}$461.1360, found 461.1362; calcd. for $\mathrm{C}_{23} \mathrm{H}_{25} \mathrm{O}_{8} \mathrm{P}[\mathrm{M}+\mathrm{Na}]^{+}$483.1179, found 483.1183 .

\section{3-O-(1-Benzyl-1H-1,2,3-triazol-4-yl)methyl-1,2-O-isopropylidene-5- $O$ - diphenylphosphono- $\alpha$-D-xylofuranose (9)}

Obtained according to the general CuI/A21 cycloaddition procedure, starting from 1,2$O$-isopropylidene-5- $O$-diphenylphosphono-3- $O$-propargyl- $\alpha$-D-xylofuranose $(8,124 \mathrm{mg}$, $0.27 \mathrm{mmol})$, benzyl azide $(0.04 \mathrm{~mL}, 0.32 \mathrm{mmol})$ in the presence of CuI/A-21 catalyst (0.06 mmol CuI, $120 \mathrm{mg}$ ). Purification by column chromatography (EtOAc/petroleum ether, 1:3) afforded the title compound $(118 \mathrm{mg}, 74 \%)$ as a colourless oil. $[\alpha]_{D}^{20}=-21$ (c = 1, in $\mathrm{CH}_{2} \mathrm{Cl}_{2}$ ). ${ }^{1} \mathrm{H}$ NMR (400 MHz, $\mathrm{CDCl}_{3}$ ): $\delta 7.54$ (s, $\left.1 \mathrm{H}, \mathrm{H}-3^{\prime}\right), 7.39-7.12$ (m, 15 $\mathrm{H}, 3 \times \mathrm{Ph}), 5.90\left(\mathrm{~d}, 1 \mathrm{H}, \mathrm{H}-1, J_{1,2}=3.6\right), 5.49,5.44\left(2 \mathrm{~d}, \mathrm{AB}\right.$ system, $2 \mathrm{H}, \mathrm{CH}_{2} \mathrm{Ph}, J_{\mathrm{a}, \mathrm{b}}=$ 15.1), 4.73 (d, part A of AB system, $\left.1 \mathrm{H}, \mathrm{H}-1^{\prime} \mathrm{a}, J_{1^{\prime} \mathrm{a}, 1^{\prime} \mathrm{b}}=11.9\right), 4.65$ (d, 1H, H-2), 4.574.33 (m, 4 H, H-1'b H-4, H-5a, H-5b), 4.01 (br. s, 1 H, H-3), 1.48 (s, 3 H, $\mathrm{CH}_{3}, i$-Pr), $1.32\left(\mathrm{~s}, 3 \mathrm{H}, \mathrm{CH}_{3}, i\right.$-Pr). ${ }^{13} \mathrm{C} \mathrm{NMR}\left(100 \mathrm{MHz} \mathrm{CDCl}_{3}\right): \delta=150.44\left(\mathrm{~d}, \mathrm{Cq}, \mathrm{Ph}, J_{\mathrm{C}, \mathrm{P}}=7.5\right)$, $150.36\left(\mathrm{~d}, \mathrm{Cq}, \mathrm{Ph}, J_{\mathrm{C}, \mathrm{P}}=7.4\right), 134.6(7(\mathrm{Cq}, \mathrm{Ph}), 129.9,129.1,128.8,128.2,125.6(\mathrm{CH}$, $3 \times \mathrm{Ph}), 123.0\left(\mathrm{C}-3^{\prime}\right) 120.12\left(\mathrm{~d}, C \mathrm{H}-o, \mathrm{Ph}, J_{\mathrm{C}-\mathrm{P}}=4.9\right), 120.10\left(\mathrm{~d}, C \mathrm{H}-o, \mathrm{Ph}, J_{\mathrm{C}-\mathrm{P}}=4.9\right)$, 112.1 (Cq, $i$-Prop), 105.3 (C-1), 81.9 (C-2), $81.4(\mathrm{C}-3), 78.2\left(\mathrm{~d}, \mathrm{C}-4, J_{\mathrm{C}-4, \mathrm{P}}=9.4\right), 65.6$ $\left(\mathrm{d}, \mathrm{C}-5, J_{\mathrm{C}-5, \mathrm{P}}=5.7\right), 63.7\left(\mathrm{C}-1^{\prime}\right), 54.2\left(\mathrm{CH}_{2} \mathrm{Ph}\right), 26.9,26.3\left(2 \times \mathrm{CH}_{3}, i-\mathrm{Pr}\right) .{ }^{31} \mathrm{P} \mathrm{NMR}$ $\left(162 \mathrm{MHz}, \mathrm{CDCl}_{3}\right): \delta-12.1 \mathrm{ppm}$. HRMS: calcd for $\mathrm{C}_{30} \mathrm{H}_{32} \mathrm{~N}_{3} \mathrm{O}_{8} \mathrm{P}[M+\mathrm{H}]^{+}$594.2000, found 594.2004.

\section{5-Azido-5-deoxy-1,2-O-isopropylidene-3- $O$-propargyl- $\alpha$-D-xylofuranose (11)}

To a solution of 5-azido-5-deoxy-1,2- $O$-isopropylidene- $\alpha$-D-xylofuranose (10, $600 \mathrm{mg}$, $2.8 \mathrm{mmol})$ in DMF $(15 \mathrm{~mL})$, at $0^{\circ} \mathrm{C}$ and under nitrogen atmosphere, sodium hydride (60\% in mineral oil, $132 \mathrm{mg}, 3.3 \mathrm{mmol}$ ) was added. After stirring during $10 \mathrm{~min}$, propargyl bromide $(0.36 \mathrm{~mL}, 3.3 \mathrm{mmol})$ was added. The reaction was stirred at room temperature during $5 \mathrm{~min}$, whereupon completed conversion was shown by TLC. Then, the mixture was diluted with diethyl ether and water was added. The phases were separated and the aqueous phase was extracted with diethyl ether $(2 \times)$. The combined organic layers were dried with anhydrous $\mathrm{MgSO}_{4}$, filtered and the solvent was evaporated in vacuum. The crude was subjected to column chromatography (EtOAc/hexane, 1:5) to afford the title compound $(673 \mathrm{mg}, 95 \%)$ as a yellow oil. $[\alpha]_{D}^{20}$ $=-25\left(\mathrm{c}=1\right.$, in $\left.\mathrm{CH}_{2} \mathrm{Cl}_{2}\right) .{ }^{1} \mathrm{H} \mathrm{NMR}\left(400 \mathrm{MHz}, \mathrm{CDCl}_{3}\right): \delta 5.88\left(\mathrm{~d}, 1 \mathrm{H}, \mathrm{H}-1, J_{1,2}=3.8\right)$,

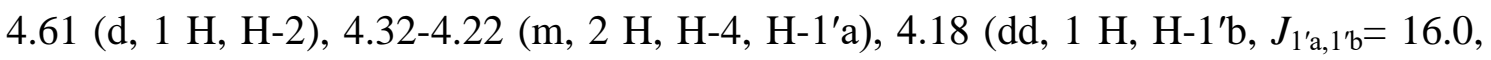
$\left.J_{1^{\prime} \mathrm{b}, 3^{\prime}}=2.4\right), 4.07\left(\mathrm{~d}, 1 \mathrm{H}, \mathrm{H}-3, J_{3,4}=3.2\right), 3.54(\mathrm{dd}$, part A of ABX system, $1 \mathrm{H}, \mathrm{H}-5 \mathrm{a}$, $\left.J_{5 \mathrm{a}, 5 \mathrm{~b}}=12.6, J_{4,5 \mathrm{a}}=6.8\right), 3.46\left(\mathrm{dd}\right.$, part B of ABX system $\left.1 \mathrm{H}, \mathrm{H}-5 \mathrm{~b}, J_{4,5 \mathrm{~b}}=6.3\right), 2.49(\mathrm{t}$, $\left.1 \mathrm{H}, \mathrm{H}-3^{\prime}, J=2.4\right), 1.47$ (s, $3 \mathrm{H}, \mathrm{CH}_{3}, i$-Pr), 1.29 (s, $3 \mathrm{H}, \mathrm{CH}_{3}, i$-Pr). ${ }^{13} \mathrm{C} \mathrm{NMR}(100$ $\mathrm{MHz}, \mathrm{CDCl} 3): \delta=112.0(\mathrm{Cq}, i$-Pr), 105.1 (C-1), $82.0(\mathrm{C}-2), 81.2$ (C-3), 78.8 (C-2'), 78.7 (C-4), $75.5\left(\mathrm{C}-3^{\prime}\right), 57.4\left(\mathrm{C}-1^{\prime}\right), 49.3(\mathrm{C}-5), 26.8,26.3\left(2 \times \mathrm{CH}_{3}, i\right.$-Pr). HRMS: calcd. for $\mathrm{C}_{11} \mathrm{H}_{15} \mathrm{~N}_{3} \mathrm{O}_{4}[\mathrm{M}+\mathrm{H}]^{+}$254.1135, found 254.1137 . 


\section{3,5'-Anhydro-5-deoxy-5-(5-hydroxymethyl-1H-1,2,3-triazol-1-yl)-1,2-O- isopropylidene- $\alpha$-D-xylofuranose (12-a)}

A solution of 5-azido-5-deoxy-1,2- $O$-isopropylidene-3- $O$-propargyl- $\alpha$-D-xylofuranose (11, $60 \mathrm{mg}, 0.23 \mathrm{mmol})$ in toluene $(5 \mathrm{~mL})$ was stirred at $110{ }^{\circ} \mathrm{C}$ for $2 \mathrm{~h}$. The solution was concentrated under vacuum, the solid was filtered off and washed with petroleum ether, affording the title compound as a white powder (40 mg, 67\%). Crystallization from EtOAc resulted in colourless monocrystals of 12-a. $[\alpha]_{D}^{20}=-12$ (c $=1$, in $\mathrm{CH}_{2} \mathrm{Cl}_{2}$ ). m.p.: 201.0-203.1 ${ }^{\circ} \mathrm{C}$. HRMS: calcd for $\mathrm{C}_{11} \mathrm{H}_{15} \mathrm{~N}_{3} \mathrm{O}_{4}[\mathrm{M}+\mathrm{H}]^{+}$254.1135, found 254.1136. NMR data were in accordance with the published data. ${ }^{27}$

\section{5-Azido-3-O-(1-benzyl-1H-1,2,3-triazol-4-yl)methyl-5-deoxy-1,2-O-isopropylidene- $\alpha$-D-xylofuranose (14)}

To a solution of 3-O-(1-benzyl-1H-1,2,3-triazol-4-yl)methyl-1,2- $O$-isopropylidene- $\alpha$-Dxylofuranose $(4,300 \mathrm{mg}, 0.83 \mathrm{mmol})$ in pyridine $(12 \mathrm{~mL})$, tosyl chloride $(320 \mathrm{mg}, 1.66$ mmol) was added. The solution was left stirring at room temperature and under nitrogen atmosphere for $16 \mathrm{~h}$. It was then diluted with ethyl acetate and washed with $2 \mathrm{M} \mathrm{HCl}$ soln. and water. The organic phase was dried with anhydrous magnesium sulfate, filtered and the solvent was evaporated under vacuum. The crude residue was dried under vacuum and then it was dissolved in DMF $(20 \mathrm{~mL})$. To the resulting solution and under nitrogen atmosphere, sodium azide (162 $\mathrm{mg}, 2.5 \mathrm{mmol})$ was added and the mixture was stirred at $80{ }^{\circ} \mathrm{C}$ for $16 \mathrm{~h}$. Then, the mixture was diluted with diethyl ether and water was added. The phases were separated and the aqueous phase was extracted with diethyl ether $(2 \times)$. The combined organic layers were dried with anhydrous $\mathrm{MgSO}_{4}$, filtered and the solvent was evaporated in vacuum. The crude was subjected to column chromatography (EtOAc/hexane, 1:2) to afford the title compound (251 mg, 78 $\%, 2$ steps) as a yellow oil. $[\alpha]_{D}^{20}=-23\left(\mathrm{c}=1\right.$, in $\left.\mathrm{CH}_{2} \mathrm{Cl}_{2}\right) .{ }^{1} \mathrm{H}$ NMR $(400 \mathrm{MHz}$, $\mathrm{CDCl}_{3}$ ): $\delta 7.45$ (s, $\left.1 \mathrm{H}, \mathrm{H}-3^{\prime}\right)$, 7.42-7.33 (m, $\left.3 \mathrm{H}, \mathrm{Ph}\right), 7.31-7.26(\mathrm{~m}, 2 \mathrm{H}, \mathrm{Ph}), 5.88$ (d, 1 $\left.\mathrm{H}, \mathrm{H}-2, J_{1,2}=3.7\right), 5.53\left(\mathrm{~s}, 2 \mathrm{H}, \mathrm{CH}_{2}, \mathrm{Bn}\right), 4.77(\mathrm{~d}, 1 \mathrm{H}$, part A of AB system, H-1'a, $\left.J_{1^{\prime} \mathrm{a}, 1^{\prime} \mathrm{b}}=12.3\right), 4.67-4.60\left(\mathrm{~m}, 2 \mathrm{H}, \mathrm{H}-1^{\prime} \mathrm{b}, \mathrm{H}-2\right), 4.28(\mathrm{td}, 1 \mathrm{H}, \mathrm{H}-4), 4.00$ (d, $1 \mathrm{H}, \mathrm{H}-3, J_{3,4}$ = 3.2), $3.49\left(\mathrm{dd}, 1 \mathrm{H}\right.$, part A of ABX system ABX, H-5a, $\left.J_{5 \mathrm{a}, 5 \mathrm{~b}}=12.3, J_{4,5 \mathrm{a}}=6.6\right), 3.41$ (dd, $1 \mathrm{H}$, part B of ABX system, H-5b, $\left.J_{4,5 \mathrm{~b}}=6.9\right), 1.49$ (s, $3 \mathrm{H}, \mathrm{CH}_{3}, i$-Pr), $1.31(\mathrm{~s}, 3 \mathrm{H}$, $\mathrm{CH}_{3}, i$-Pr). ${ }^{13} \mathrm{C}$ NMR (100 MHz, $\left.\mathrm{CDCl}_{3}\right): \delta 144.6\left(\mathrm{C}-2^{\prime}\right), 134.4(\mathrm{Cq}, \mathrm{Ph}), 129.2,128.9$, $128.1(\mathrm{CH}, \mathrm{Ph}), 122.6$ (C-3'), 112.0 (Cq, $i$-Pr), 105.1 (C-1), 82.0 (C-2), 81.7 (C-3), 78.5 (C-4), 63.4 (C-1'), 54.3 ( $\left.\mathrm{CH}_{2}, \mathrm{Bn}\right), 49.0$ (C-5), 26.8, 26.2 (2 × CH3, $i$-Pr). HRMS: calcd. for $\mathrm{C}_{18} \mathrm{H}_{22} \mathrm{~N}_{6} \mathrm{O}_{4}[\mathrm{M}+\mathrm{H}]^{+} 387.1775$, found 387.1779; calcd. for $\mathrm{C}_{18} \mathrm{H}_{22} \mathrm{~N}_{6} \mathrm{O}_{4}[\mathrm{M}+\mathrm{Na}]^{+}$ 409.1595, found 409.1599 .

\section{3-O-(1-Benzyl-1H-1,2,3-triazol-4-yl)methyl-5-deoxy-5-(diethylphosphono)amino- 1,2-O-isopropylidene- $\alpha$-D-xylofuranose (14)}

To a solution of 5-azido-3-O-(1-benzyl-1H-1,2,3-triazol-4-yl)methyl-5-deoxy-1,2-Oisopropylidene- $\alpha$-D-xylofuranose $(\mathbf{1 3}, 30 \mathrm{mg}, 0.08 \mathrm{mmol})$ in $\mathrm{CH}_{2} \mathrm{Cl}_{2}(5 \mathrm{~mL})$, triethyl 
phosphite $(0.07 \mathrm{~mL}, 0.39 \mathrm{mmol})$ was added. The reaction was stirred at room temperature for $48 \mathrm{~h}$. The solution was concentrated under vaccum and the residue was subjected to column chromatography (EtOAc/MeOH, 20:1) to afford the title compound (37 $\mathrm{mg}, 96 \%)$ as a colourless oil.

$[\alpha]_{D}^{20}=-3.6\left(\mathrm{c}=1\right.$, in $\left.\mathrm{CH}_{2} \mathrm{Cl}_{2}\right) .{ }^{1} \mathrm{H} \mathrm{NMR}\left(400 \mathrm{MHz}, \mathrm{CDCl}_{3}\right): \delta 7.45\left(\mathrm{~s}, 1 \mathrm{H}, \mathrm{H}-3^{\prime}\right)$, 7.40-7.32 (m, $3 \mathrm{H}, \mathrm{Ph}), 7.30-7.24(\mathrm{~m}, 2 \mathrm{H}, \mathrm{Ph}), 5.87\left(\mathrm{~d}, 1 \mathrm{H}, J_{1,2}=3.8\right), 5.51(\mathrm{~s}, 2 \mathrm{H}$, $\left.\mathrm{CH}_{2} \mathrm{Ph}\right), 4.76\left(\mathrm{~d}, 1 \mathrm{H}\right.$, part A of AB system, H-1'a, $\left.J_{1^{\prime} \mathrm{a}, 1^{\prime} \mathrm{b}}=12.4\right), 4.63-4.54(\mathrm{~m}, 2 \mathrm{H}, \mathrm{H}-$ $\left.1^{\prime} \mathrm{b}, \mathrm{H}-2\right), 4.25\left(\mathrm{td}, 1 \mathrm{H}, \mathrm{H}-4, J_{4,5 \mathrm{a}}=J_{4,5 \mathrm{~b}}=6.7, J_{3,4}=3.3\right), 4.08-3.94(\mathrm{~m}, 5 \mathrm{H}, \mathrm{H}-3,2 \times$ $\left.\mathrm{CH}_{2}, \mathrm{Et}\right), 3.35\left(\mathrm{dt}, 1 \mathrm{H}, \mathrm{NH}, J_{\mathrm{NH}, \mathrm{P}}=10.7, J_{\mathrm{NH}, 5 \mathrm{a}}=J_{\mathrm{NH}, 5 \mathrm{~b}}=7.3\right), 3.24-3.06$ (m, $2 \mathrm{H}, \mathrm{H}-5 \mathrm{a}$, $\mathrm{H}-5 \mathrm{~b}$ ), 1.46 (s, $3 \mathrm{H}, \mathrm{CH}_{3}, i$-Pr), 1.32-1.24 (m, $3 \mathrm{H}, \mathrm{CH}_{3}, i$-Pr, $2 \times \mathrm{CH}_{3}, \mathrm{Et}$ ). ${ }^{13} \mathrm{C} \mathrm{NMR}$ $\left(100 \mathrm{MHz}, \mathrm{CDCl}_{3}\right): \delta 144.6\left(\mathrm{C}-2^{\prime}\right), 134.5(\mathrm{Cq}, \mathrm{Ph}), 129.3,128.9,128.3(\mathrm{CH}, \mathrm{Ph}), 122.5$ (C-3'), $111.8\left(\mathrm{Cq}, i\right.$-Pr), 105.1 (C-1), $82.1(\mathrm{C}-2), 82.0(\mathrm{C}-3), 79.8\left(\mathrm{~d}, \mathrm{C}-4, J_{\mathrm{C}-4, \mathrm{P}}=5.1\right)$, $63.2\left(\mathrm{C}-1^{\prime}\right), 62.47,62.44\left(2 \mathrm{~d}, 2 \times \mathrm{CH}_{2}, \mathrm{Et}, J_{\mathrm{CH} 2, \mathrm{P}}=5.3\right), 54.3\left(\mathrm{CH}_{2}, \mathrm{Bn}\right), 39.7(\mathrm{C}-5)$, 26.8, $26.4\left(2 \times \mathrm{CH}_{3}, i\right.$-Pr), $16.3\left(\mathrm{~d}, 2 \times \mathrm{CH}_{3}, \mathrm{Et}, J_{\mathrm{C}, \mathrm{P}}=7.2\right) .{ }^{31} \mathrm{P} \mathrm{NMR}(162 \mathrm{MHz}$, $\left.\mathrm{CDCl}_{3}\right): \delta$ 9.11. HRMS: calcd. for $\mathrm{C}_{22} \mathrm{H}_{33} \mathrm{~N}_{4} \mathrm{O}_{7} \mathrm{P}[\mathrm{M}+\mathrm{H}]^{+}$497.2160, found 497.2176; calcd. for $\mathrm{C}_{22} \mathrm{H}_{33} \mathrm{~N}_{4} \mathrm{O}_{7} \mathrm{P}[\mathrm{M}+\mathrm{Na}]^{+}$519.1979, found 519.1990 .

\section{5-Bromo-5-deoxy-1,2- $O$-isopropylidene-3- $O$-propargyl- $\alpha$-D-xylofuranose (15)}

To a solution of 1,2- $O$-isopropylidene-3- $O$-propargyl- $\alpha$-D-xylofuranose $(3,100 \mathrm{mg}$, $0.44 \mathrm{mmol})$ in $\mathrm{CH}_{2} \mathrm{Cl}_{2}(10 \mathrm{~mL})$ under nitrogen, triphenilphosphine (230 mg, $\left.0.88 \mathrm{mmol}\right)$ and carbon tetrabromide ( $292 \mathrm{mg}, 0.88 \mathrm{mmol}$ ) were added. The mixture was stirred at room temperature and under nitrogen atmosphere for $24 \mathrm{~h}$. The mixture was concentrated under vacuum and the crude residue was subjected to column chromatography (EtOAc/hexane, 1:5) to afford the title compound (30 mg, 24\%) as a colourless oil.

$[\alpha]_{D}^{20}=-37\left(\mathrm{c}=1\right.$, in $\left.\mathrm{CH}_{2} \mathrm{Cl}_{2}\right) .{ }^{1} \mathrm{H} \mathrm{NMR}\left(400 \mathrm{MHz}, \mathrm{CDCl}_{3}\right): \delta 5.92\left(\mathrm{~d}, 1 \mathrm{H}, \mathrm{H}-1, J_{1,2}=\right.$ 3.6), 4.68 (d, $1 \mathrm{H}, \mathrm{H}-2), 4.46$ (ddd, $1 \mathrm{H}, \mathrm{H}-4), 4.34-4.24$ (br.s, $2 \mathrm{H}, \mathrm{H}-1^{\prime} \mathrm{a}, \mathrm{H}-1$ 'b, $J_{1^{\prime} \mathrm{a}, 1^{\prime} \mathrm{b}}=$ $\left.16.1, J_{1^{\prime} \mathrm{a}, 3^{\prime}}=2.3, J_{1^{\prime} \mathrm{b}, 3^{\prime}}=2.3\right), 4.17\left(\mathrm{~d}, 1 \mathrm{H}, \mathrm{H}-3, J_{3,4}=2.9\right), 3.57-3.49$ (m, $2 \mathrm{H}, \mathrm{H}-5 \mathrm{a}, \mathrm{H}-$ $\left.5 \mathrm{~b}, J_{5 \mathrm{a}, 5 \mathrm{~b}}=9.7, J_{4,5 \mathrm{a}}=8.7, J_{4,5 \mathrm{a}}=5.9\right), 2.50\left(\mathrm{~m}, 1 \mathrm{H}, \mathrm{H}-3^{\prime}, J=2.3\right), 1.51\left(\mathrm{~s}, 3 \mathrm{H}, \mathrm{CH}_{3}, i-\right.$ Pr), 1.33 (s,3 H, $\mathrm{CH}_{3}, i$-Pr). ${ }^{13} \mathrm{C} \mathrm{NMR}\left(100 \mathrm{MHz}, \mathrm{CDCl}_{3}\right): \delta 112.3(\mathrm{Cq}, i$-Pr), $105.6(\mathrm{C}-$ 1), 82.3 (C-2), 81.1 (C-3), 80.4 (C-4), $79.0\left(\mathrm{C}-2^{\prime}\right), 75.4\left(\mathrm{C}-33^{\prime}\right), 58.3\left(\mathrm{C}-1^{\prime}\right), 27.0\left(\mathrm{CH}_{3}, i-\right.$ Pr), 26.9 (C-5), $26.4\left(\mathrm{CH}_{3}, i\right.$-Pr). HRMS: calcd. for $\mathrm{C}_{11} \mathrm{H}_{15} \mathrm{BrO}_{4}[\mathrm{M}+\mathrm{Na}]^{+}$313.0046, found 313.0040.

\section{5-Azido-3- $O$-benzyl-5-deoxy-1,2- $O$-isopropylidene- $\alpha$-D-xylofuranose (18)}

To a solution of 3- $O$-benzyl-1,2- $O$-isopropylidene- $\alpha$-D-xylofuranose $\left(\mathbf{1 6},{ }^{13 \mathrm{e}} 785 \mathrm{mg}, 2.8\right.$ $\mathrm{mmol})$ in pyridine $(17 \mathrm{~mL})$, tosyl chloride $(640 \mathrm{mg}, 3.4 \mathrm{mmol})$ was added. The solution was left stirring at room temperature and under nitrogen atmosphere for $16 \mathrm{~h}$. It was then diluted with ethyl acetate and washed with $2 \mathrm{M} \mathrm{HCl}$ soln. and water. The organic phase was dried with anhydrous magnesium sulfate, filtered and the solvent was evaporated under vacuum. The crude residue was dried under vacuum and then it was 
dissolved in DMF (12 mL). To the resulting solution and under nitrogen atmosphere, sodium azide ( $546 \mathrm{mg}, 8.4 \mathrm{mmol}$ ) was added and the mixture was stirred at $80{ }^{\circ} \mathrm{C}$ for 16 $\mathrm{h}$. Then, the mixture was diluted with diethyl ether and water was added. The phases were separated and the aqueous phase was extracted with diethyl ether $(2 \times)$. The combined organic layers were dried with anhydrous $\mathrm{MgSO}_{4}$, filtered and the solvent was evaporated in vacuum. The crude was subjected to column chromatography (EtOAc/hexane, 1:7) to afford the title compound (684 mg, 80\%, 2 steps) as a colourless oil. The NMR data were in accordance with the reported data. ${ }^{33}$

General procedure for the thermal azide-alkyne cycloaddition between 5-azido-3$O$-protected 1,2- $O$-isopropylidene- $\alpha$-D-xylofuranoses and propargyl derivatives

To a solution of 5-azido-3- $O$-protected 1,2- $O$-isopropylidene- $\alpha$-D-xylofuranose (1 $\mathrm{mmol})$ in toluene $(7 \mathrm{~mL})$ under nitrogen, propargyl alcohol, propargylamine or propargyl bromide ( 2 equiv.) was added. The reaction mixture was stirred under reflux for 16-48 h. After concentration under vacuum, the residue was subjected to column chromatography.

\section{3-O-Benzyl-5-deoxy-5-(4-hydroxymethyl-1H-1,2,3-triazol-1-yl)-1,2-O-}

\section{isopropylidene- $\alpha$-D-xylofuranose $\quad(20) \quad$ and $\quad 3-O$-benzyl-5-deoxy-5-(5-} hydroxymethyl-1H-1,2,3-triazol-1-yl)-1,2-O-isopropylidene- $\alpha$-D-xylofuranose (21)

Obtained according to the general procedure for thermal azide-alkyne cycloaddition, starting from 5-azido-3- $O$-benzyl-5-deoxy-1,2- $O$-isopropylidene- $\alpha$-D-xylofuranose (18, $300 \mathrm{mg}, 0.98 \mathrm{mmol})$ and propargyl alcohol $(0.11 \mathrm{~mL}, 1.96 \mathrm{mmol})$. The reaction was stirred for $24 \mathrm{~h}$. Purification by column chromatography (EtOAc/Hex, 1:1) afforded the 1,4-disubstituted triazole $\mathbf{2 0}(163 \mathrm{mg}, 46 \%)$ as colourless crystals and the 1,5disubstituted regioisomer $\mathbf{2 1}(126 \mathrm{mg}, 36 \%)$ as a colourless oil.

Data for 20: $[\alpha]_{D}^{20}=-40\left(\mathrm{c}=1\right.$, in $\left.\mathrm{CH}_{2} \mathrm{Cl}_{2}\right) .{ }^{1} \mathrm{H} \mathrm{NMR}\left(400 \mathrm{MHz}, \mathrm{CDCl}_{3}\right): \delta 7.60(\mathrm{~s}, 1$ H, H-5), 7.43-7.29 (m, 5 H, Ph), 5.98 (d, 1 H, H-1', $J_{1^{\prime}, 2^{\prime}}=3.7$ ), 4.80-4.60 (m, 5 H, H-a, from $\mathrm{CH}_{2} \mathrm{Ph}, \mathrm{CH}_{2} \mathrm{OH}, \mathrm{H}-2^{\prime}, \mathrm{H}-5^{\prime} \mathrm{a}$ ), 4.59-4.46 (m, $3 \mathrm{H}, \mathrm{H}-4^{\prime}, \mathrm{H}-\mathrm{b}$ from $\mathrm{CH}_{2} \mathrm{Ph}, \mathrm{H}-5^{\prime} \mathrm{b}$ ), 4.00 (d, $\left.1 \mathrm{H}, \mathrm{H}-3^{\prime}, J_{3^{\prime}, 4^{\prime}}=2.7\right), 1.43$ (s, $3 \mathrm{H}, \mathrm{CH}_{3}, i$-Pr.), 1.31 (s, $3 \mathrm{H}, \mathrm{CH}_{3}, i$-Pr.). ${ }^{13} \mathrm{C}$ NMR (100 MHz, $\left.\mathrm{CDCl}_{3}\right): \delta 147.9(\mathrm{C}-4), 136.8(\mathrm{Cq}, \mathrm{Ph}), 128.7,128.3,128.0(\mathrm{CH}, \mathrm{Ph})$, 123.0 (C-5), 112.1 (Cq, i-Pr.), 105.2 (C-1'), 81.9 (C-2'), 81.5 (C-3'), 78.8 (C-4'), 71.9 $\left(\mathrm{CH}_{2} \mathrm{Ph}\right) 56.0\left(\mathrm{CH}_{2} \mathrm{OH}\right), 49.2\left(\mathrm{C}-5^{\prime}\right), 26.7,26.2\left(2 \times \mathrm{CH}_{3}, i\right.$-Pr $)$. HRMS: calcd. for $\mathrm{C}_{18} \mathrm{H}_{23} \mathrm{~N}_{3} \mathrm{O}_{5}[\mathrm{M}+\mathrm{H}]^{+} 362.1710$, found 362.1716; calcd. for $[\mathrm{M}+\mathrm{Na}]^{+} 384.1530$, found 384.1535 .

Data for 21: $[\alpha]_{D}^{20}=-32\left(\mathrm{c}=1\right.$, in $\left.\mathrm{CH}_{2} \mathrm{Cl}_{2}\right) .{ }^{1} \mathrm{H} \mathrm{NMR}\left(400 \mathrm{MHz}, \mathrm{CDCl}_{3}\right): \delta 7.58(\mathrm{~s}, 1$ $\mathrm{H}, \mathrm{H}-4), 7.44-7.27$ (m, $5 \mathrm{H}, \mathrm{Ph}), 5.99$ (d, 1H, H-1', $J_{1^{\prime}, 2^{\prime}}=3.7$ ), 4.74-4.49 (m, $8 \mathrm{H}, \mathrm{H}-2^{\prime}$, $\left.\mathrm{H}-4^{\prime}, \mathrm{CH}_{2} \mathrm{Ph}, \mathrm{CH}_{2} \mathrm{OH}, \mathrm{CH}_{2}-5^{\prime}, J_{\mathrm{a}, \mathrm{b}(\mathrm{Bn})}=11.8\right), 4.10\left(\mathrm{~d}, 1 \mathrm{H}, \mathrm{H}-3^{\prime}, J_{3,4}=1.8\right), 1.41(\mathrm{~s}, 3$ $\mathrm{H}, \mathrm{CH}_{3}, i$-Pr), $1.30\left(\mathrm{~s}, 3 \mathrm{H}, \mathrm{CH}_{3}, i\right.$-Pr). ${ }^{13} \mathrm{C} \mathrm{NMR}\left(100 \mathrm{MHz}, \mathrm{CDCl}_{3}\right): \delta 137.3(\mathrm{C}-5)$, 
$136.8(\mathrm{Cq}, \mathrm{Ph}), 133.3(\mathrm{C}-4), 128.7,128.4,128.0(\mathrm{CH}, \mathrm{Ph}), 123.0\left(\mathrm{C}-1^{\prime}\right), 112.4(\mathrm{Cq}, i-$ Pr), $105.1\left(\mathrm{C}-1^{\prime}\right), 82.0\left(\mathrm{C}-2^{\prime}\right), 81.7\left(\mathrm{C}-3^{\prime}\right), 79.6\left(\mathrm{C}^{\prime} 4^{\prime}\right), 72.1\left(\mathrm{CH}_{2} \mathrm{Ph}\right) 52.6\left(\mathrm{CH}_{2} \mathrm{OH}\right)$, $47.7\left(\mathrm{C}-5^{\prime}\right), 26.7,26.2\left(2 \times \mathrm{CH}_{3}, i\right.$-Pr $)$. HRMS: calcd. for $\mathrm{C}_{18} \mathrm{H}_{23} \mathrm{~N}_{3} \mathrm{O}_{5}[\mathrm{M}+\mathrm{H}]^{+}$ 362.1710, found 362.1715; calcd. for $[\mathrm{M}+\mathrm{Na}]^{+} 384.1530$, found 384.1537.

\section{5-Deoxy-3-O-dodecyl-5-(4-hydroxymethyl-1H-1,2,3-triazol-1-yl)-1,2-O-}

\section{isopropylidene- $\alpha$-D-xylofuranose (22) and 5-deoxy-3-O-dodecyl-5-(5-} hydroxymethyl-1H-1,2,3-triazol-1-yl)-1,2-O-isopropylidene- $\alpha$-D-xylofuranose (23)

Obtained according to the general procedure for thermal azide-alkyne cycloaddition, starting from 5-azido-5-deoxy-3- $O$-dodecyl-1,2- $O$-isopropylidene- $\alpha$-D-xylofuranose (19, $400 \mathrm{mg}, 1.04 \mathrm{mmol})$ and propargyl alcohol $(0.12 \mathrm{~mL}, 2.09 \mathrm{mmol})$. The reaction was stirred for $48 \mathrm{~h}$. Purification by column chromatography (EtOAc/Hex, 1:3) afforded the 1,4-disubstituted triazole 22 (248 $\mathrm{mg}, 54 \%)$ and the 1,5-disubstituted regioisomer $\mathbf{2 3}$ (143 $\mathrm{mg}, 31 \%)$ as colourless oils.

Data for 22: $[\alpha]_{D}^{20}=-17\left(\mathrm{c}=1\right.$, in $\left.\mathrm{CH}_{2} \mathrm{Cl}_{2}\right) \cdot{ }^{1} \mathrm{H}$ NMR $\left(400 \mathrm{MHz}, \mathrm{CDCl}_{3}\right): \delta 7.65(\mathrm{~s}, 1$ H, H-5), 5.93 (d, $1 \mathrm{H}, \mathrm{H}-1^{\prime}, J_{1^{\prime}, 2^{\prime}}=3.6$ ), 4.78-4.69 (m, $3 \mathrm{H}, \mathrm{CH}_{2} \mathrm{OH}, \mathrm{H}-5^{\prime} \mathrm{a}, J_{5^{\prime} \mathrm{a}, 5^{\prime} \mathrm{b}}=18.9$, $\left.J_{4,5^{\prime} \mathrm{a}}=8.4\right), 4.58\left(\mathrm{~d}, 1 \mathrm{H}, \mathrm{H}-2^{\prime}, J_{2^{\prime}, 1^{\prime}}=3.6\right), 4.54-4.43$ (m, $\left.2 \mathrm{H}, \mathrm{H}-4^{\prime}, \mathrm{H}-5{ }^{\prime} \mathrm{b}\right), 3.84$ (d, $1 \mathrm{H}$, H-3', $\left.J_{3^{\prime}, 4^{\prime}}=2.0\right), 3.63\left(\mathrm{dt}, 1 \mathrm{H}, \mathrm{H}-1^{\prime \prime} \mathrm{a}, J_{1^{\prime \prime} \mathrm{a}, 1^{\prime \prime} \mathrm{b}}=9.1, J_{1^{\prime \prime} \mathrm{a}, 2^{\prime \prime a}}=J_{1^{\prime \prime} \mathrm{a}, 2^{\prime \prime} \mathrm{b}}=6.5\right), 3.41(\mathrm{dt}$, $\left.1 \mathrm{H}, \mathrm{H}-1^{\prime \prime} \mathrm{b}, J_{1^{\prime \prime} \mathrm{b}, 2^{\prime \prime \mathrm{a}}}=J_{1^{\prime \prime} \mathrm{b}, 2^{\prime \prime} \mathrm{b}}=6.7\right), 1.63-1.49\left(\mathrm{~m}, 2 \mathrm{H}, \mathrm{H}-2^{\prime \prime}\right), 1.41\left(\mathrm{~s}, 3 \mathrm{H}, \mathrm{CH}_{3}, i-\mathrm{Pr}\right)$, 1.36-1.16 (m, $21 \mathrm{H}, \mathrm{CH}_{3}, i-\mathrm{Pr}, \mathrm{H}-3$ " to $\left.\mathrm{H}-11^{\prime \prime}\right), 0.86\left(\mathrm{t}, 3 \mathrm{H}, \mathrm{CH}_{3}-12^{\prime \prime}, J=6.8\right) .{ }^{13} \mathrm{C}$ NMR (100 MHz, $\left.\mathrm{CDCl}_{3}\right): \delta 147.9(\mathrm{C}-4), 123.0(\mathrm{C}-5), 112.1(\mathrm{Cq}, i-\mathrm{Pr}), 105.3\left(\mathrm{C}-1^{\prime}\right)$, $82.6\left(\mathrm{C}-3^{\prime}\right), 82.1\left(\mathrm{C}-3^{\prime}\right), 79.2\left(\mathrm{C}-4^{\prime}\right), 70.6\left(\mathrm{C}-1^{\prime \prime}\right), 56.5\left(\mathrm{CH}_{2} \mathrm{OH}\right), 49.5\left(\mathrm{C}-5^{\prime}\right), 32.0,29.8$, 29.8, 29.7, 29.7, 29.7, 29.5, 29.5, 26.3 (C-2" to C-10"), 26.8, $26.2\left(2 \times \mathrm{CH}_{3}, i\right.$-Pr), 22.8 $\left(\mathrm{C}-11^{\prime \prime}\right), 14.2\left(\mathrm{C}-3^{\prime \prime}\right)$. HRMS: calcd. for $\mathrm{C}_{23} \mathrm{H}_{41} \mathrm{~N}_{3} \mathrm{O}_{5}[\mathrm{M}+\mathrm{H}]^{+} 440.3119$, found 440.3125; calcd. for $[\mathrm{M}+\mathrm{Na}]^{+} 462.2938$, found 462.2941 .

Data for 23: $[\alpha]_{D}^{20}=-25\left(\mathrm{c}=1\right.$, in $\left.\mathrm{CH}_{2} \mathrm{Cl}_{2}\right) .{ }^{1} \mathrm{H} \mathrm{NMR}\left(400 \mathrm{MHz}, \mathrm{CDCl}_{3}\right): \delta 7.60(\mathrm{~s}, 1$ H, H-4), 5.92 (d, $1 \mathrm{H}, \mathrm{H}-1^{\prime}, J_{1^{\prime}, 2^{\prime}}=3.5$ ), 4.77-4.65 (m, $\left.3 \mathrm{H}, \mathrm{CH}_{2} \mathrm{OH}, \mathrm{H}-5^{\prime} \mathrm{a}\right), 4.61-4.45$ (m, $3 \mathrm{H}, \mathrm{H}-2^{\prime}, \mathrm{H}-4^{\prime}, \mathrm{H}-5^{\prime} \mathrm{b}, J_{5^{\prime} \mathrm{a}, 5^{\prime} \mathrm{b}}=14.2, J_{4,5^{\prime} \mathrm{b}}=8.6$ ), 3.94 (d, $\left.1 \mathrm{H}, \mathrm{H}-3^{\prime}, J_{3^{\prime}, 4^{\prime}}=3.0\right), 3.63$ $\left(\mathrm{dt}, 1 \mathrm{H}, \mathrm{H}-1^{\prime \prime} \mathrm{a}, J_{1 " \mathrm{a}, 1 " \mathrm{~b}}=9.1, J_{1 " \mathrm{a}, 2^{\prime \prime} \mathrm{a}}=J_{1^{\prime \prime} \mathrm{a}, 2^{\prime \prime} \mathrm{b}}=6.6\right), 3.43\left(\mathrm{dt}, 1 \mathrm{H}, \mathrm{H}-1{ }^{\prime \prime} \mathrm{b}, J_{1 " \mathrm{~b}, 2^{\prime \prime} \mathrm{a}}=\right.$ $\left.J_{1^{\prime \prime} \mathrm{b}, 2^{\prime \prime} \mathrm{b}}=6.7\right), 1.60-1.49\left(\mathrm{~m}, 2 \mathrm{H}, \mathrm{CH}_{2}-2^{\prime \prime}\right), 1.38$ (s, $\left.3 \mathrm{H}, \mathrm{CH}_{3}, i-\mathrm{Pr}\right), 1.34-1.16$ (m, $21 \mathrm{H}$, $\mathrm{CH}_{3}, i$-Pr, $\mathrm{CH}_{2}-3$ " to $\left.\mathrm{CH}_{2}-11^{\prime \prime}\right), 0.85$ (t, $3 \mathrm{H}, \mathrm{CH}_{3}-12$ ",$\left.J=6.5\right) .{ }^{13} \mathrm{C} \mathrm{NMR}(100 \mathrm{MHz}$, $\left.\mathrm{CDCl}_{3}\right): \delta=137.2(\mathrm{C}-5), 133.6(\mathrm{C}-4), 112.5$ (Cq, $i$-Pr), $105.2\left(\mathrm{C}-1^{\prime}\right), 82.8\left(\mathrm{C}-3^{\prime}\right), 82.1$ $\left(\mathrm{C}-2^{\prime}\right), 80.0\left(\mathrm{C}^{4} 4^{\prime}\right), 70.8\left(\mathrm{C}-1^{\prime \prime}\right), 52.6\left(\mathrm{CH}_{2} \mathrm{OH}\right), 47.9\left(\mathrm{C}-5^{\prime}\right), 32.0,29.7,29.7,29.7,29.5$, 29.4, 26.3 (C-2" to $\left.\mathrm{C}-10^{\prime \prime}\right), 26.8,26.2\left(2 \times \mathrm{CH}_{3}, i\right.$-Pr), $22.8\left(\mathrm{C}-11^{\prime \prime}\right), 14.2\left(\mathrm{C}-2^{\prime \prime}\right)$. HRMS: calcd. for $\mathrm{C}_{23} \mathrm{H}_{41} \mathrm{~N}_{3} \mathrm{O}_{5}[\mathrm{M}+\mathrm{H}]^{+} 440.3119$, found 440.3130; calcd. for [M+ $\mathrm{Na}]^{+}$462.2938, found 462.2953. 
General procedure for the phosphorylation of 3-O-protected 5-(hydroxymethyl$1 H$-1,2,3-triazol-1-yl)-1,2- $O$-isopropylidene- $\alpha$-D-xylofuranoses with diethyl phosphorochloridate

To a solution of 5-(hydroxymethyl-1H-1,2,3-triazol-1-yl)-xylofuranose derivative (0.14 $\mathrm{mmol})$ in dichloromethane $(3 \mathrm{~mL})$ and under nitrogen atmosphere, triethylamine $(1.5$ equiv.) was added. The reaction mixture was cooled to $0^{\circ} \mathrm{C}$ and diethyl phosphorochloridate (1.5 equiv) and DMAP (catalytic amount, one spatula point) were added. The reaction was stirred at room temperature overnight. The solution was concentrated under vacuum and the residue was purified by column chromatography.

\section{3-O-Benzyl-5-deoxy-5-[4-(diethoxyphosphoryloxy)methyl-1H-1,2,3-triazol-1-yl]- 1,2-O-isopropylidene- $\alpha$-D-xylofuranose (24)}

According to the general procedure, the treatment of 3-O-benzyl-5-deoxy-5-(4hydroxymethyl-1 $H$-1,2,3-triazol-1-yl)-1,2- $O$-isopropylidene- $\alpha$-D-xylofuranose $\quad(20,50$ $\mathrm{mg}, \quad 0.14 \mathrm{mmol})$ with triethylamine $(0.03 \mathrm{~mL}, 0.21 \mathrm{mmol})$ and diethyl phosphorochloridate $(0.03 \mathrm{~mL}, 0.21 \mathrm{mmol})$ and purification by flash column chromatography (EtOAc /Hexane, 1:2) afforded the title compound (54 mg, 78\%) as a colourless oil.

$[\alpha]_{D}^{20}=-20\left(\mathrm{c}=1\right.$, in $\left.\mathrm{CH}_{2} \mathrm{Cl}_{2}\right) .{ }^{1} \mathrm{H}$ NMR $\left(400 \mathrm{MHz}, \mathrm{CDCl}_{3}\right): \delta 7.72(\mathrm{~s}, 1 \mathrm{H}, \mathrm{H}-5), 7.43-$ $7.30(\mathrm{~m}, 5 \mathrm{H}, \mathrm{Ph}), 5.97\left(\mathrm{~d}, 1 \mathrm{H}, \mathrm{H}-1^{\prime}, J_{1^{\prime}, 2^{\prime}}=3.6\right), 5.16\left(\mathrm{~d}, 2 \mathrm{H}, \mathrm{CH}_{2} \mathrm{OP}, J_{\mathrm{CH} 2, \mathrm{P}}=8.8\right), 4.74$ (d, part A of AB system, $\left.1 \mathrm{H}, \mathrm{H}-\mathrm{a}, \mathrm{CH}_{2} \mathrm{Ph}, J_{\mathrm{a}, \mathrm{b}}=11.7\right)$, 4.70-4.62 (m, $2 \mathrm{H}, \mathrm{H}-2$ ', H-5'a), 4.59-4.49 (m, 3 H, H-5'b, H-4', H-b from $\mathrm{CH}_{2} \mathrm{Ph}$ ), 4.15-4.04 (m, $4 \mathrm{H}, 2 \times \mathrm{CH}_{2}, 2 \times \mathrm{Et}$ ), $3.99\left(\mathrm{~d}, 1 \mathrm{H}, \mathrm{H}-3^{\prime}, J_{3^{\prime}, 4^{\prime}}=2.2\right), 1.42\left(\mathrm{~s}, 3 \mathrm{H}, \mathrm{CH}_{3}, i-\mathrm{Pr}\right), 1.35-1.27\left(\mathrm{~m}, 9 \mathrm{H}, 2 \times \mathrm{CH}_{3}, 2 \times\right.$ Et, $\mathrm{CH}_{3}, i$-Pr) ppm. ${ }^{13} \mathrm{C}$ NMR (100 MHz, $\left.\mathrm{CDCl}_{3}\right): \delta 143.4\left(\mathrm{~d}, \mathrm{C}-4, J_{\mathrm{C}, \mathrm{P}}=7.5\right), 136.9$ $(\mathrm{Cq}, \mathrm{Ph}), 128.9,128.5,128.1(\mathrm{CH}, \mathrm{Ph}), 124.8$ (C-5), $112.3\left(\mathrm{Cq}, i\right.$-Prop), $105.4\left(\mathrm{C}-1^{\prime}\right)$, $82.1\left(\mathrm{C}-2^{\prime}\right), 81.6\left(\mathrm{C}-3^{\prime}\right), 78.9\left(\mathrm{C}-4^{\prime}\right), 72.1,\left(\mathrm{CH}_{2}, \mathrm{Bn}\right), 64.1\left(\mathrm{~d}, 2 \times \mathrm{CH}_{2}, 2 \times \mathrm{Et}, J_{\mathrm{C}, \mathrm{P}}=\right.$ 5.9), $60.6\left(\mathrm{~d}, \mathrm{CH}_{2} \mathrm{OP}, J_{\mathrm{C}, \mathrm{P}}=5.1\right), 49.6\left(\mathrm{C}-5^{\prime}\right), 26.9,26.3\left(2 \times \mathrm{CH}_{3}, i-\mathrm{Pr}\right), 16.1(\mathrm{~d}, 2 \times$ $\left.\mathrm{CH}_{3}, 2 \times \mathrm{Et}, J_{\mathrm{C}, \mathrm{P}}=6.9\right) .{ }^{31} \mathrm{P} \mathrm{NMR}\left(162 \mathrm{MHz}, \mathrm{CDCl}_{3}\right): \delta=-1.15$. HRMS: calcd. for $\mathrm{C}_{22} \mathrm{H}_{32} \mathrm{~N}_{3} \mathrm{O}_{8} \mathrm{P}[\mathrm{M}+\mathrm{H}]^{+}$498.2000, found 498.1995; calcd. for $[\mathrm{M}+\mathrm{Na}]^{+}$520.1819, found 520.1814 .

\section{3-O-Benzyl-5-deoxy-5-[5-(diethoxyphosphoryloxy)methyl-1H-1,2,3-triazol-1-yl]-}

\section{1,2-O-isopropylidene- $\alpha$-D-xylofuranose (25)}

According to the general procedure, the treatment of 3-O-benzyl-5-deoxy-5-(5hydroxymethyl-1 $H$-1,2,3-triazol-1-yl)-1,2- $O$-isopropylidene- $\alpha$-D-xylofuranose $(\mathbf{2 1}, 50$ $\mathrm{mg}, \quad 0.14 \mathrm{mmol})$ with triethylamine $(0.03 \mathrm{~mL}, 0.21 \mathrm{mmol})$ and diethyl phosphorochloridate $(0.03 \mathrm{~mL}, 0.21 \mathrm{mmol})$ and purification by flash column chromatography (EtOAc/hexane, 1:2) afforded the title compound (47 mg, 68\%) as a colourless oil.

$[\alpha]_{D}^{20}=-23\left(\mathrm{c}=1\right.$, in $\left.\mathrm{CH}_{2} \mathrm{Cl}_{2}\right) .{ }^{1} \mathrm{H}$ NMR $\left(400 \mathrm{MHz}, \mathrm{CDCl}_{3}\right): \delta 7.70(\mathrm{~s}, 1 \mathrm{H}, \mathrm{H}-4), 7.42-$ $7.28(\mathrm{~m}, 5 \mathrm{H}, \mathrm{Ph}), 5.95\left(\mathrm{~d}, 1 \mathrm{H}, \mathrm{H}-1^{\prime}, J_{1^{\prime}, 2^{\prime}}=3.6\right), 5.24(\mathrm{dd}, 1 \mathrm{H}$, part A of ABX system, 
$\mathrm{H}$-a from $\left.\mathrm{CH}_{2} \mathrm{OP}, J_{\mathrm{a}, \mathrm{b}}=13.1, J_{\mathrm{H}-\mathrm{a}, \mathrm{P}}=9.2\right) 5.11(\mathrm{dd}$, part B of ABX system, $1 \mathrm{H}, \mathrm{H}-\mathrm{b}$ from $\left.\mathrm{CH}_{2} \mathrm{OP}, J_{\mathrm{H}-\mathrm{b}, \mathrm{P}}=8.4\right)$, 4.76-4.55 (m, $5 \mathrm{H}, \mathrm{H}-\mathrm{a}$ from $\mathrm{CH}_{2} \mathrm{Ph}, \mathrm{CH}_{2}-5^{\prime}, \mathrm{H}-2^{\prime}, \mathrm{H}-4^{\prime}, J_{\mathrm{a}, \mathrm{b}}$ = 11.7), $4.50\left(\mathrm{~d}, 1 \mathrm{H}, \mathrm{H}-\mathrm{b}\right.$ from $\left.\mathrm{CH}_{2} \mathrm{Ph}\right), 4.11-3.96\left(\mathrm{~m}, 5 \mathrm{H}, 2 \times \mathrm{CH}_{2}, 2 \times \mathrm{Et}, \mathrm{H}-3^{\prime}\right), 1.40$ $\left(\mathrm{s}, 3 \mathrm{H}, \mathrm{CH}_{3}, i\right.$-Pr), 1.32-1.20 (m, $9 \mathrm{H}, 2 \times \mathrm{CH}_{3}$ from $2 \times \mathrm{Et}, \mathrm{CH}_{3}, i$-Pr). ${ }^{13} \mathrm{C} \mathrm{NMR}(100$ $\mathrm{MHz}_{\mathrm{CDCl}}$ ): $\delta 136.9(\mathrm{Cq}, \mathrm{Ph}), 134.3(\mathrm{C}-4), 133.0\left(\mathrm{~d}, \mathrm{C}-5, J_{\mathrm{C}-5, \mathrm{P}}=7.8\right)$, 128.8, 128.5,

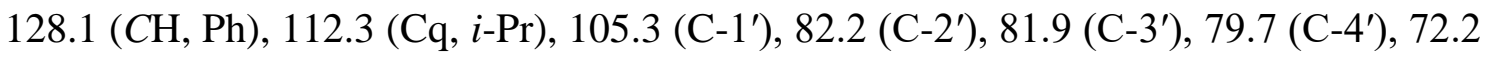
$\left(\mathrm{CH}_{2} \mathrm{Ph}\right), 64.3\left(\mathrm{~d}, 2 \times \mathrm{CH}_{2}, 2 \times \mathrm{Et}, J_{\mathrm{C}, \mathrm{P}}=5.9\right), 57.0\left(\mathrm{~d}, C \mathrm{H}_{2} \mathrm{OP}, J_{\mathrm{C}, \mathrm{P}}=4.9\right), 47.9\left(\mathrm{C}-5^{\prime}\right)$, 26.8, $26.3\left(2 \times \mathrm{CH}_{3}, i\right.$-Pr) $16.2\left(\mathrm{~d}, 2 \times \mathrm{CH}_{3}, 2 \times \mathrm{Et}, J_{\mathrm{C}, \mathrm{P}}=6.8\right) .{ }^{31} \mathrm{P} \mathrm{NMR}(162 \mathrm{MHz}$, $\mathrm{CDCl}_{3}$ ): $\delta-1.17$. HRMS: calcd. for $\mathrm{C}_{22} \mathrm{H}_{32} \mathrm{~N}_{3} \mathrm{O}_{8} \mathrm{P}[\mathrm{M}+\mathrm{H}]^{+}$498.2000, found 498.2010; calcd. for $[\mathrm{M}+\mathrm{Na}]^{+}$520.1819, found 520.1830.

\section{5-Deoxy-5-[4-(diethoxyphosphoryloxy)methyl-3-O-dodecyl-1H-1,2,3-triazol-1-yl]-}

\section{1,2-O-isopropylidene- $\alpha$-D-xylofuranose (26)}

According to the general procedure, the treatment of 5-deoxy-3-O-dodecyl-5-(4hydroxymethyl-1H-1,2,3-triazol-1-yl)-1,2- $O$-isopropylidene- $\alpha$-D-xylofuranose $\quad(22,50$ $\mathrm{mg}, \quad 0.11 \mathrm{mmol})$ with triethylamine $(0.02 \mathrm{~mL}, 0.17 \mathrm{mmol})$ and diethyl phosphorochloridate $(0.02 \mathrm{~mL}, 0.17 \mathrm{mmol})$ and purification by column chromatography (EtOAc/Hexane, 1:2) afforded the title compound (53 $\mathrm{mg}, 81 \%)$ as a colourless oil. $[\alpha]_{D}^{20}=-15\left(\mathrm{c}=1\right.$, in $\left.\mathrm{CH}_{2} \mathrm{Cl}_{2}\right) .{ }^{1} \mathrm{H}$ NMR $\left(400 \mathrm{MHz}, \mathrm{CDCl}_{3}\right): \delta 7.80(\mathrm{~s}, 1 \mathrm{H}, \mathrm{H}-5), 5.93$ $\left(\mathrm{d}, 1 \mathrm{H}, \mathrm{H}-1^{\prime} J_{1^{\prime}, 2^{\prime}}=3.6\right), 5.18\left(\mathrm{~d}, 2 \mathrm{H}, \mathrm{CH}_{2} \mathrm{OP}, J_{\mathrm{CH} 2, \mathrm{P}}=8.8\right), 4.72(\mathrm{dd}$, part A of ABX system, $\left.1 \mathrm{H}, \mathrm{H}-5^{\prime} \mathrm{a}, J_{4^{\prime}, 5^{\prime} \mathrm{a}}=7.5, J_{5^{\prime} \mathrm{a}, 5^{\prime} \mathrm{b}}=17.5\right), 4.59\left(\mathrm{~d}, 1 \mathrm{H}, \mathrm{H}-2^{\prime}\right), 4.56-4.45$ (m, $2 \mathrm{H}, \mathrm{H}-$ $\left.4^{\prime}, \mathrm{H}-5^{\prime} \mathrm{b}\right), 4.15-4.04$ (m, $4 \mathrm{H}, 2 \times \mathrm{CH}_{2}, 2 \times \mathrm{Et}$ ), 3.85 (d, $\left.1 \mathrm{H}, \mathrm{H}-3^{\prime}, J_{3^{\prime}, 4^{\prime}}=2.4\right), 3.64$ (dt, 1 $\left.\mathrm{H}, \mathrm{H}-1{ }^{\prime \prime} \mathrm{a}, J_{1 " \mathrm{a}, 2 \mathrm{a}}=J_{1 " \mathrm{a}, 2^{\prime \prime} \mathrm{b}}=6.5, J_{1 " \mathrm{a}, 1^{\prime \prime} \mathrm{b}}=9.2\right), 3.43\left(\mathrm{dt}, 1 \mathrm{H}, \mathrm{H}-1^{\prime \prime} \mathrm{b}, J_{1 " \mathrm{~b}, 2^{\prime \prime a}}=J_{1 " \mathrm{~b}, 2^{\prime \prime} \mathrm{b}}=\right.$ 6.6), 1.62-1.52 (m, $\left.2 \mathrm{H}, \mathrm{CH}_{2}-2^{\prime \prime}\right), 1.42$ (s, $3 \mathrm{H}, \mathrm{CH}_{3}, i$-Pr), 1.38-1.18 (m, $27 \mathrm{H}, 2 \times \mathrm{CH}_{3}$, $2 \times \mathrm{Et}, \mathrm{CH}_{3}, i$-prop, $\mathrm{CH}_{2}-3^{\prime \prime}$ to $\left.\mathrm{CH}_{2}-11^{\prime \prime}\right), 0.90$ (t, $\left.3 \mathrm{H}, \mathrm{CH}_{3}-12^{\prime \prime}, J=6.6\right) .{ }^{13} \mathrm{C} \mathrm{NMR} \mathrm{(100}$ $\left.\mathrm{MHz}, \mathrm{CDCl}_{3}\right): \delta 143.4\left(\mathrm{~d}, \mathrm{C}-4, J_{\mathrm{C}-4, \mathrm{P}}=7.3\right), 124.8(\mathrm{C}-5), 112.2\left(\mathrm{Cq}, i\right.$-Pr), $105.4\left(\mathrm{C}-1^{\prime}\right)$, $82.6\left(\mathrm{C}-3^{\prime}\right), 82.2\left(\mathrm{C}-2^{\prime}\right), 79.0\left(\mathrm{C}-4^{\prime}\right), 70.7\left(\mathrm{C}-1^{\prime \prime}\right), 64.1\left(\mathrm{~d}, 2 \times \mathrm{CH}_{2}, 2 \times\right.$ Et $\left.J_{\mathrm{C}, \mathrm{P}}=5.9\right)$, $60.6\left(\mathrm{~d}, \mathrm{CH}_{2} \mathrm{OP}, J_{\mathrm{C}, \mathrm{P}}=5.2\right), 49.6\left(\mathrm{C}-5^{\prime}\right), 32.1,29.8,29.8,29.8,29.7,29.6,29.5,26.3(\mathrm{C}-$ $2^{\prime \prime}$ to $\left.\mathrm{C}-10^{\prime \prime}\right), 26.9,26.3\left(2 \times \mathrm{CH}_{3}, i\right.$-Pr), $\left.22.8 \mathrm{C}-11^{\prime \prime}\right), 16.2\left(\mathrm{~d}, 2 \times \mathrm{CH}_{3}, 2 \times \mathrm{Et}, J_{\mathrm{C}, \mathrm{P}}=\right.$ 6.8), $14.3\left(\mathrm{C}-12^{\prime \prime}\right) .{ }^{31} \mathrm{P}$ NMR $\left(162 \mathrm{MHz}, \mathrm{CDCl}_{3}\right): \delta-1.13$. HRMS: calcd. for $\mathrm{C}_{27} \mathrm{H}_{50} \mathrm{~N}_{3} \mathrm{O}_{8} \mathrm{P}[\mathrm{M}+\mathrm{H}]^{+}$576.3408, found 576.3405; calcd. for $[\mathrm{M}+\mathrm{Na}]^{+}$598.3228, found 598.3224 .

\section{5-Deoxy-5-[5-(diethoxyphosphoryloxy)methyl-3-O-dodecyl-1H-1,2,3-triazol-1-yl]- 1,2-O-isopropylidene- $\alpha$-D-xylofuranose (27)}

According to the general procedure, the treatment of 5-deoxy-3-O-dodecyl-5-(5hydroxymethyl-1 $H$-1,2,3-triazol-1-yl)-1,2- $O$-isopropylidene- $\alpha$-D-xylofuranose $(\mathbf{2 3}, 50$ $\mathrm{mg}, \quad 0.11 \mathrm{mmol})$ with triethylamine $(0.02 \mathrm{~mL}, 0.17 \mathrm{mmol})$ and diethyl phosphorochloridate $(0.02 \mathrm{~mL}, 0.17 \mathrm{mmol})$ and purification by column chromatography (EtOAc/Hexane, 1:2) afforded the title compound (61 mg, 93\%) as a colourless oil. 
$[\alpha]_{D}^{20}=-10\left(\mathrm{c}=1\right.$, in $\left.\mathrm{CH}_{2} \mathrm{Cl}_{2}\right) .{ }^{1} \mathrm{H} \mathrm{NMR}\left(400 \mathrm{MHz}, \mathrm{CDCl}_{3}\right): \delta 7.72(\mathrm{~s}, 1 \mathrm{H}, \mathrm{H}-4), 5.92$ $\left(\mathrm{d}, 1 \mathrm{H}, \mathrm{H}-1^{\prime} J_{1^{\prime}, 2^{\prime}}=3.6\right), 5.29\left(\mathrm{dd}\right.$, part A of ABX system, $1 \mathrm{H}, \mathrm{H}$-a from $\mathrm{CH}_{2} \mathrm{OP}, J_{\mathrm{a}, \mathrm{b}}=$ $\left.13.2, J_{\mathrm{H}-\mathrm{a}, \mathrm{P}}=9.2\right), 5.17$ (dd, part B of ABX system, $1 \mathrm{H}, \mathrm{H}-\mathrm{b}$ from $\mathrm{CH}_{2} \mathrm{OP}, J_{\mathrm{H}-\mathrm{b}, \mathrm{P}}=8.4$ ), 4.73 (dd, 1 H, H-5'a, $J_{4^{\prime}, 5^{\prime} \mathrm{a}}=8.3, J_{\mathrm{a}, \mathrm{b}}=19.3$ ), 4.61-4.50 (m, $\left.3 \mathrm{H}, \mathrm{H}-2^{\prime}, \mathrm{H}^{\prime} 4^{\prime}, \mathrm{H}-5{ }^{\prime} \mathrm{b}\right), 4.15-$ $4.01\left(\mathrm{~m}, 4 \mathrm{H}, 2 \times \mathrm{CH}_{2}, 2 \times \mathrm{Et}\right), 3.92\left(\mathrm{~d}, 1 \mathrm{H}, \mathrm{H}-3^{\prime}, J_{3^{\prime}, 4^{\prime}}=2.1\right), 3.63\left(\mathrm{dt}, 1 \mathrm{H}, \mathrm{H}-1^{\prime \prime} \mathrm{a}\right.$,

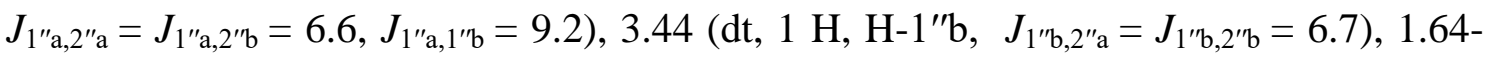
1.49 (m, $\left.2 \mathrm{H}, \mathrm{CH}_{2}-2^{\prime \prime}\right), 1.41$ (s, $\left.3 \mathrm{H}, \mathrm{CH}_{3}, i-\mathrm{Pr}\right), 1.37-1.19$ (m, $27 \mathrm{H}, 2 \times \mathrm{CH}_{3}, 2 \times \mathrm{Et}$, $\mathrm{CH}_{3}, i$-Pr, $\mathrm{CH}_{2}-3^{\prime \prime}$ to $\left.\mathrm{CH}_{2}-11^{\prime \prime}\right), 0.87\left(\mathrm{t}, 3 \mathrm{H}, \mathrm{CH}_{3}-12^{\prime \prime}, J=6.5\right) .{ }^{13} \mathrm{C}$ NMR $(100 \mathrm{MHz}$, $\left.\mathrm{CDCl}_{3}\right): \delta 134.4(\mathrm{C}-4), 133.2\left(\mathrm{~d}, \mathrm{C}-5, J_{\mathrm{C}-5, \mathrm{P}}=7.8\right), 112.2(\mathrm{Cq}, i-\mathrm{P}), 105.3\left(\mathrm{C}-1^{\prime}\right), 82.7$ (C-3'), $82.2\left(\mathrm{C}-2^{\prime}\right), 79.8\left(\mathrm{C}-4^{\prime}\right), 70.7\left(\mathrm{C}-1^{\prime \prime}\right), 64.4\left(\mathrm{~d}, 2 \times \mathrm{CH}_{2}, 2 \times \mathrm{Et}, J_{\mathrm{C}, \mathrm{P}}=5.9\right), 57.1$ $\left(\mathrm{d}, \mathrm{CH}_{2} \mathrm{OP}, J_{\mathrm{C}, \mathrm{P}}=5.1\right), 48.0\left(\mathrm{C}-5^{\prime}\right), 32.1,29.8,29.8,29.7,29.6,29.5,26.4\left(\mathrm{C}-2^{\prime \prime}\right.$ to C$\left.\left.10^{\prime \prime}\right), 26.8,26.3\left(2 \times \mathrm{CH}_{3}, i-\mathrm{Pr}\right), 22.8 \mathrm{C}-11^{\prime \prime}\right), 16.2\left(\mathrm{~d}, 2 \times \mathrm{CH}_{3}, 2 \times \mathrm{Et}, J_{\mathrm{C}, \mathrm{P}}=6.8\right), 14.3$ $\left(\mathrm{C}-12^{\prime \prime}\right) .{ }^{31} \mathrm{P}$ NMR $\left(162 \mathrm{MHz}, \mathrm{CDCl}_{3}\right): \delta-1.13 \mathrm{ppm}$. HRMS: calcd. for $\mathrm{C}_{27} \mathrm{H}_{50} \mathrm{~N}_{3} \mathrm{O}_{8} \mathrm{P}$ $[\mathrm{M}+\mathrm{H}]^{+} 576.3408$, found 576.3405 ; calcd. for $[\mathrm{M}+\mathrm{Na}]^{+}$598.3228, found 598.3224 .

\section{5-(4-Aminomethyl-1H-1,2,3-triazol-1-yl)-3-O-benzyl-5-deoxy-1,2- $O$-isopropylidene- $\alpha$-D-xylofuranose (28) and 5-(5-aminomethyl-1H-1,2,3-triazol-1-yl)-3-O-benzyl-5- deoxy-1,2- $O$-isopropylidene- $\alpha$-D-xylofuranose (29)}

Obtained according to the general procedure for thermal azide-alkyne cycloaddition, starting from 5-azido-3-O-benzyl-5-deoxy-1,2- $O$-isopropylidene- $\alpha$-D-xylofuranose (18, $600 \mathrm{mg}, 1.97 \mathrm{mmol})$ and propargylamine $(0.25 \mathrm{~mL}, 3.93 \mathrm{mmol})$. The reaction was stirred for 24 h. Purification by column chromatography (from EtOAc to EtOAc/MeOH, 18:1) afforded the 1,4-disubstituted triazole $22(20 \mathrm{mg}, 3 \%)$ as a yellow oil and the 1,5disubstituted regioisomer 29 (20 mg, 3\%, not isolated from 18), along with recovered 18 (480 mg, 80\%).

Data for 28: $[\alpha]_{D}^{20}=-12\left(\mathrm{c}=1\right.$, in $\left.\mathrm{CH}_{2} \mathrm{Cl}_{2}\right) .{ }^{1} \mathrm{H} \mathrm{NMR}\left(400 \mathrm{MHz}, \mathrm{CDCl}_{3}\right): \delta 7.61(\mathrm{~s}, 1$ $\mathrm{H}, \mathrm{H}-5), 7.42-7.28$ (m, $5 \mathrm{H}, \mathrm{Ph}), 5.95$ (d, 1H, H-1', $J_{1^{\prime}, 2^{\prime}}=3.3$ ), 4.76-4.58 (m, $3 \mathrm{H}, \mathrm{H}-2^{\prime}$, $\mathrm{H}-\mathrm{a}$ from $\mathrm{CH}_{2} \mathrm{Ph}, \mathrm{H}-5$ 'a), 4.57-4.43 (m, $3 \mathrm{H}, \mathrm{H}-4{ }^{\prime}, \mathrm{H}-\mathrm{b}$ from $\mathrm{CH}_{2} \mathrm{Ph}, \mathrm{H}-5$ 'b), 4.07-3.93 (m, $\left.3 \mathrm{H}, \mathrm{CH}_{2} \mathrm{~N}, \mathrm{H}-3^{\prime}\right), 1.40$ (s, $3 \mathrm{H}, \mathrm{CH}_{3}, i$-Pr), 1.29 (s, 3H, $\mathrm{CH}_{3}, i$-Prop). ${ }^{13} \mathrm{C}$ NMR (100 $\mathrm{MHz}, \mathrm{CDCl}_{3}$ ): $\delta 146.9(\mathrm{C}-4), 137.0(\mathrm{Cq}, \mathrm{Ph}), 128.8,128.4,128.1(\mathrm{CH}, \mathrm{Ph}), 123.0(\mathrm{C}-5)$, $112.2\left(\mathrm{Cq}, i\right.$-Pr), $105.3\left(\mathrm{C}-1^{\prime}\right), 82.1\left(\mathrm{C}-2^{\prime}\right), 81.6\left(\mathrm{C}-3^{\prime}\right), 79.0\left(\mathrm{C}-4^{\prime}\right), 72.1\left(\mathrm{CH}_{2} \mathrm{Ph}\right), 49.4$ $\left(\mathrm{C}-5^{\prime}\right), 36.8\left(\mathrm{CH}_{2} \mathrm{~N}\right), 26.8,26.3\left(2 \times \mathrm{CH}_{3}, i\right.$-Pr). HRMS: calcd. for $\mathrm{C}_{18} \mathrm{H}_{24} \mathrm{~N}_{4} \mathrm{O}_{4}[\mathrm{M}+\mathrm{H}]^{+}$ 361.1870, found 361.1870; calcd. for $\mathrm{C}_{18} \mathrm{H}_{24} \mathrm{~N}_{4} \mathrm{O}_{4}[\mathrm{M}+\mathrm{Na}]^{+} 383.1690$, found 383.1690.

NMR data for 29: ${ }^{1} \mathrm{H}$ NMR (400 MHz, $\left.\mathrm{CDCl}_{3}\right): \delta 7.56$ (s, $\left.1 \mathrm{H}, \mathrm{H}-4\right), 7.41-7.28(\mathrm{~m}, 5$ $\mathrm{H}, \mathrm{Ph}$ ), 5.96 (d, 1H, H-1', $J_{1^{\prime}, 2^{\prime}}=3.7$ ), 4.79-4.57 (m, $4 \mathrm{H}, \mathrm{H}-\mathrm{a}$ from $\mathrm{CH}_{2} \mathrm{Ph}, \mathrm{H}-2^{\prime}, \mathrm{H}-4^{\prime}$, H-5'a), 4.55-4.43 (m, 2 H, H-b from $\mathrm{CH}_{2} \mathrm{Ph}, \mathrm{H}-5^{\prime} \mathrm{b}$ ), 4.07 (d, $1 \mathrm{H}, \mathrm{H}-3^{\prime}, J_{3^{\prime}, 4^{\prime}}=3.1$ ), 3.94 $\left(\mathrm{s}, 2 \mathrm{H}, \mathrm{CH}_{2} \mathrm{~N}\right), 1.41$ (s, $\left.3 \mathrm{H}, \mathrm{CH}_{3}, i-\mathrm{Pr}\right), 1.29$ (s, $3 \mathrm{H}, \mathrm{CH}_{3}, i$-Pr). ${ }^{*}{ }^{13} \mathrm{C} \mathrm{NMR}(100 \mathrm{MHz}$, $\left.\mathrm{CDCl}_{3}\right): \delta 139.3(\mathrm{C}-5), 137.0(\mathrm{Cq}, \mathrm{Ph}), 132.4(\mathrm{C}-4), 128.8,128.5,128.1(\mathrm{CH}, \mathrm{Ph}), 112.3$ 


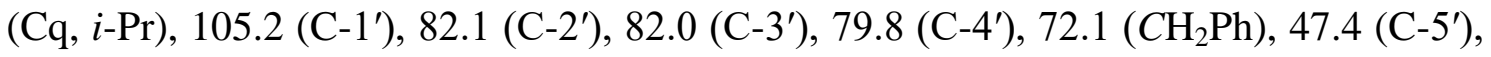
$35.0\left(\mathrm{CH}_{2} \mathrm{~N}\right), 26.8,26.3\left(2 \times \mathrm{CH}_{3}, \mathrm{i}-\mathrm{Pr}\right){ }^{*}$

*Data extracted from the spectrum of a mixture containing 18/29.

\section{5-(4-Aminomethyl-1H-1,2,3-triazol-1-yl)-5-deoxy-3-O-dodecyl-1,2-O-}

isopropylidene- $\alpha$-D-xylofuranose (30) and 5-(5-aminomethyl-1H-1,2,3-triazol-1-yl)5-deoxy-3-O-dodecyl-1,2- $O$-isopropylidene- $\alpha$-D-xylofuranose (31)

Obtained according to the general procedure for thermal azide-alkyne cycloaddition, starting from 5-azido-5-deoxy-3- $O$-dodecyl-1,2- $O$-isopropylidene- $\alpha$-D-xylofuranose $(19,600 \mathrm{mg}, 1.56 \mathrm{mmol})$ and propargylamine $(0.2 \mathrm{~mL}, 3.12 \mathrm{mmol})$. The reaction was stirred for $16 \mathrm{~h}$. Purification by column chromatography (from EtOAc to EtOAc/MeOH, 18:1) afforded the 1,4-disubstituted triazole $\mathbf{3 0}$ (140 mg, 20\%) and the 1,5-disubstituted regioisomer 31 (140 mg, 20\%) as yellow oils, along with recovered 19 (239 mg, 40\%).

Data for 30: $[\alpha]_{D}^{20}=-45\left(\mathrm{c}=1\right.$, in $\left.\mathrm{CH}_{2} \mathrm{Cl}_{2}\right) .{ }^{1} \mathrm{H}$ NMR $\left(400 \mathrm{MHz}, \mathrm{CDCl}_{3}\right): \delta 7.67(\mathrm{~s}, 1$ $\mathrm{H}, \mathrm{H}-5), 5.93\left(\mathrm{~d}, 1 \mathrm{H}, \mathrm{H}-1^{\prime}, J_{1^{\prime}, 2^{\prime}}=3.6\right), 4.69$ (dd, $1 \mathrm{H}$, part A of ABX system, H-5'a, $\left.J_{4^{\prime}, 5^{\prime} \mathrm{a}}=8.9, J_{5^{\prime} \mathrm{a}, 5^{\prime} \mathrm{b}}=20.9\right) 4.58\left(\mathrm{~d}, 1 \mathrm{H}, \mathrm{H}-2^{\prime}, J_{2^{\prime}, 1^{\prime}}=3.6\right), 4.56-4.42\left(\mathrm{~m}, 2 \mathrm{H}, \mathrm{H}-55^{\prime} \mathrm{b}, \mathrm{H}-4^{\prime}\right)$, $4.04\left(\mathrm{~s}, 2 \mathrm{H}, \mathrm{CH}{ }_{2} \mathrm{~N}\right), 3.85\left(\mathrm{~d}, 1 \mathrm{H}, \mathrm{H}-3, J_{3^{\prime}, 4^{\prime}}=2.4\right) 3.63\left(\mathrm{dt}, 1 \mathrm{H}, \mathrm{H}-1^{\prime \prime} \mathrm{a}, J_{1^{\prime \prime} \mathrm{a}, 2^{\prime \prime} \mathrm{a}}=J_{1^{\prime \prime} \mathrm{a}, 2^{\prime \prime} \mathrm{b}}=\right.$ $\left.6.7, J_{1^{\prime \prime}, 1^{\prime \prime} \mathrm{b}}=9.1\right), 3.43\left(\mathrm{dt}, 1 \mathrm{H}, \mathrm{H}-1^{\prime \prime} \mathrm{b}, J_{1^{\prime \prime} \mathrm{b}, 2^{\prime \prime} \mathrm{a}}=J_{1^{\prime \prime} \mathrm{b}, 2^{\prime \prime} \mathrm{b}}=6.6\right), 1.66-1.55\left(\mathrm{~m}, 2 \mathrm{H}, \mathrm{CH}_{2^{-}}\right.$ $2^{\prime \prime}$ ), 1.38 (s, $3 \mathrm{H}, \mathrm{CH}_{3}, i$-Prop), 1.26-1.22 (m, $21 \mathrm{H}, \mathrm{CH}_{3}, i$-Pr, $\mathrm{CH}_{2}-3$ " to $\mathrm{CH}_{2}-11^{\prime \prime}$ ), 0.87 (t, $3 \mathrm{H}, \mathrm{CH}_{3}-12$ ", $\left.\left.J=6.9\right) .{ }^{13} \mathrm{C} \mathrm{NMHz}, \mathrm{CDCl}_{3}\right): \delta 147.8(\mathrm{C}-4), 122.4(\mathrm{C}-5), 112.0$ (Cq, $i$-Pr), $105.2\left(\mathrm{C}-1^{\prime}\right), 82.5$ (C-3'), $82.0\left(\mathrm{C}-2^{\prime}\right), 79.0$ (C-4'), $70.5\left(\mathrm{C}-1^{\prime \prime}\right), 49.3\left(\mathrm{C}-5^{\prime}\right)$, $36.9\left(\mathrm{CH}_{2} \mathrm{~N}\right), 31.9,29.7,29.6,29.6,29.6,29.4,29.3,26.2\left(\mathrm{C}-2^{\prime \prime}\right.$ to $\left.\mathrm{C}-10^{\prime \prime}\right), 26.7,26.1$ (2 $\times \mathrm{CH}_{3}, i$-Pr), $22.7\left(\mathrm{C}-11^{\prime \prime}\right), 14.1\left(\mathrm{C}-12^{\prime \prime}\right)$. HRMS: calcd for $\mathrm{C}_{23} \mathrm{H}_{42} \mathrm{~N}_{4} \mathrm{O}_{4}[\mathrm{M}+\mathrm{H}]^{+}$ 439.3279, found 439.3280 .

Data for 31: $[\alpha]_{D}^{20}=-10\left(\mathrm{c}=1\right.$, in $\left.\mathrm{CH}_{2} \mathrm{Cl}_{2}\right) .{ }^{1} \mathrm{H}$ NMR $\left(400 \mathrm{MHz}, \mathrm{CDCl}_{3}\right): \delta 7.57(\mathrm{~s}, 1$ H, H-4), 5.92 (d, 1 H, H-1', $J_{1^{\prime}, 2^{\prime}}=3.5$ ), 4.73-4.55 (m, 3 H, H-5'a, H-4', H-2', $J_{4^{\prime}, 5^{\prime} \mathrm{a}}=3.2$, $\left.J_{5^{\prime} \mathrm{a}, 5^{\prime} \mathrm{b}}=14.2\right), 4.45\left(\mathrm{dd}\right.$, part B of ABX system $\left.1 \mathrm{H}, \mathrm{H}-5^{\prime} \mathrm{b}, J_{4^{\prime}, 5^{\prime} \mathrm{b}}=7.9\right), 3.98(\mathrm{~s}, 2 \mathrm{H}$, $\left.\mathrm{CH}_{2} \mathrm{~N}\right), 3.93\left(\mathrm{~d}, 1 \mathrm{H}, J_{3^{\prime}, 4^{\prime}}=3.2\right), 3.64\left(\mathrm{dt}, 1 \mathrm{H}, \mathrm{H}-1^{\prime \prime} \mathrm{a}, J_{1^{\prime \prime} \mathrm{a}, 2^{\prime \prime} \mathrm{a}}=J_{1^{\prime \prime} \mathrm{a}, 2^{\prime \prime} \mathrm{b}}=6.6, J_{1^{\prime \prime} \mathrm{a}, 1^{\prime \prime} \mathrm{b}}=\right.$ 9.2), 3.44 (dt, $\left.1 \mathrm{H}, \mathrm{H}-1^{\prime \prime} \mathrm{b}, J_{1^{\prime \prime} \mathrm{b}, 2^{\prime \prime} \mathrm{a}}=J_{1^{\prime \prime} \mathrm{b}, 2^{\prime \prime} \mathrm{b}}=6.7\right), 1.63-1.49$ (m, $\left.2 \mathrm{H}, \mathrm{CH}_{2}-2^{\prime \prime}\right), 1.41$ (s, 3 $\mathrm{H}, \mathrm{CH}_{3}, i$-Pr), $1.38-1.16\left(\mathrm{~m}, 21 \mathrm{H}, \mathrm{CH}_{3}, i\right.$-Prop, $\mathrm{CH}_{2}-3^{\prime \prime}$ to $\left.\mathrm{CH}_{2}-11^{\prime \prime}\right), 0.87$ (t, $3 \mathrm{H}, \mathrm{CH}_{3^{-}}$ $12 ", J=6.9) .{ }^{13} \mathrm{C}$ NMR (100 MHz, $\left.\mathrm{CDCl}_{3}\right): \delta 139.2(\mathrm{C}-5), 132.4(\mathrm{C}-4), 112.2(\mathrm{Cq}, i-$ Pr), 105.3 (C-1'), 82.9 (C-3'), 82.2 (C-2'), 80.0 (C-4'), 70.7 (C-1'), 47.5 (C-5'), 35.0 $\left(\mathrm{CH}_{2} \mathrm{~N}\right), 32.1,29.8,29.8,29.7,29.5,29.5,26.3\left(\mathrm{C}-2^{\prime \prime}\right.$ to $\left.\mathrm{C}-10^{\prime \prime}\right), 26.8,26.2\left(2 \times \mathrm{CH}_{3}, i-\right.$ Pr), $22.8\left(\mathrm{C}-11^{\prime \prime}\right), \quad 14.3\left(\mathrm{C}-12^{\prime \prime}\right)$. HRMS: calcd for $\mathrm{C}_{23} \mathrm{H}_{42} \mathrm{~N}_{4} \mathrm{O}_{4}[\mathrm{M}+\mathrm{H}]^{+} 439.3279$, found 439.3280 . 
General procedure for the N-phosphorylation of 3-O-protected 5-(aminomethyl$1 H$-1,2,3-triazol-1-yl)-1,2- $O$-isopropylidene- $\alpha$-D-xylofuranoses with diethyl phosphorochloridate.

To a solution of 5-(aminomethyl-1H-1,2,3-triazol-1-yl) xylofuranose derivative $(0.35$ $\mathrm{mmol})$ in dichloromethane $(5 \mathrm{~mL})$ at $0{ }^{\circ} \mathrm{C}$ and under nitrogen atmosphere, diethyl phosphorochloridate (1.2 equiv.) was added. The mixture was stirred at room temperature for $2 \mathrm{~h}$. The solution was concentrated under vacuum and the residue was subjected to column chromatography.

\section{3-O-Benzyl-5-deoxy-5-[4-(diethylphosphono)aminomethyl-1H-1,2,3-triazol-1-yl]- 1,2-O-isopropylidene- $\alpha$-D-xylofuranose (32)}

Obtained according to the general procedure, starting from 5-(4-aminomethyl-1H-1,2,3triazol-1-yl)-3-O-benzyl-5-deoxy-1,2- $O$-isopropylidene- $\alpha$-D-xylofuranose $(\mathbf{2 8}, 30 \mathrm{mg}$, $0.08 \mathrm{mmol})$ and diethyl phosphorochloridate $(0.01 \mathrm{~mL}, 0.1 \mathrm{mmol})$. Purification by column chromatography (from EtOAc to EtOAc/MeOH, 25:1, then 18:1) afforded the title compound $(23 \mathrm{mg}, 56 \%)$ as a colourless oil.

$[\alpha]_{D}^{20}=-32\left(\mathrm{c}=1\right.$, in $\left.\mathrm{CH}_{2} \mathrm{Cl}_{2}\right) .{ }^{1} \mathrm{H}$ NMR $\left(400 \mathrm{MHz}, \mathrm{CDCl}_{3}\right): \delta 7.56(\mathrm{~s}, 1 \mathrm{H}, \mathrm{H}-5), 7.43-$ $7.30(\mathrm{~m}, 5 \mathrm{H}, \mathrm{Ph}), 5.99\left(\mathrm{~d}, 1 \mathrm{H}, \mathrm{H}-1^{\prime}, J_{1^{\prime}, 2^{\prime}}=3.6\right), 4.74(\mathrm{~d}, 1 \mathrm{H}$, part A of AB system, H-a from $\left.\mathrm{CH}_{2} \mathrm{Ph}, J_{\mathrm{a}, \mathrm{b}}=11.7\right)$, 4.70-4.60 (m, 2H, H-2', H-5'a), 4.56-4.51 (m, $3 \mathrm{H}, \mathrm{H}-\mathrm{b}$ from $\left.\mathrm{CH}_{2} \mathrm{Ph}, \mathrm{H}-4^{\prime}, \mathrm{H}-5^{\prime} \mathrm{b}\right), 4.19\left(\mathrm{dd}, 2 \mathrm{H}, \mathrm{CH}_{2} \mathrm{NH}, J_{\mathrm{CH} 2, \mathrm{P}}=10.6, J_{\mathrm{CH} 2, \mathrm{NH}}=6.8\right), 4.13-3.95(\mathrm{~m}$, $\left.5 \mathrm{H}, \mathrm{H}-3{ }^{\prime}, 2 \times \mathrm{CH}_{2}, 2 \times \mathrm{Et}\right), 3.13\left(\mathrm{dt}, 1 \mathrm{H}, \mathrm{NH}, J_{\mathrm{N} H, \mathrm{P}}=10.2\right), 1.41\left(\mathrm{~s}, 3 \mathrm{H}, \mathrm{CH}_{3}, i\right.$-Pr), 1.34-1.18 (m, $9 \mathrm{H}, \mathrm{CH}_{3}, i$-Prop, $\left.2 \times \mathrm{CH}_{3}, 2 \times \mathrm{Et}\right) .{ }^{13} \mathrm{C} \mathrm{NMR}\left(100 \mathrm{MHz}, \mathrm{CDCl}_{3}\right): \delta 146.7$ $\left(\mathrm{d}, \mathrm{C}-4, J_{\mathrm{C}, \mathrm{P}}=6.3\right), 136.9(\mathrm{Cq}, \mathrm{Ph}), 128.9,128.5,128.1(\mathrm{CH}, \mathrm{Ph}), 122.8(\mathrm{C}-5), 112.2$ (Cq, $i$-Prop), $105.4\left(\mathrm{C}-1 '^{\prime}\right), 82.1\left(\mathrm{C}-3^{\prime}\right), 81.7\left(\mathrm{C}-3^{\prime}\right), 79.0\left(\mathrm{C}-4^{\prime}\right), 71.9\left(\mathrm{CH}_{2} \mathrm{Ph}\right), 62.7$ (d, 2 $\left.\times \mathrm{CH}_{2}, 2 \times \mathrm{Et}, J_{\mathrm{C}, \mathrm{P}}=5.5\right), 49.6\left(\mathrm{C}-5^{\prime}\right), 37.0\left(\mathrm{CH}_{2} \mathrm{~N}\right), 26.8,26.3\left(2 \times \mathrm{CH}_{3}, i-\mathrm{Pr}\right), 16.3(\mathrm{~d}$, $\left.2 \times \mathrm{CH}_{3}, 2 \times \mathrm{Et}, J_{\mathrm{C}, \mathrm{P}}=7.0\right) .{ }^{31} \mathrm{P} \mathrm{NMR}\left(162 \mathrm{MHz}, \mathrm{CDCl}_{3}\right): \delta$ 8.19. HRMS: calcd. for $\mathrm{C}_{22} \mathrm{H}_{33} \mathrm{~N}_{4} \mathrm{O}_{7} \mathrm{P}[\mathrm{M}+\mathrm{H}]^{+}$497.2160, found 497.2173; calcd. for $[\mathrm{M}+\mathrm{Na}]^{+}$519.1979, found 519.1997 .

\section{5-Deoxy-3-O-dodecyl-5-[4-(diethylphosphono)aminomethyl-1H-1,2,3-triazol-1-yl]-}

\section{1,2-O-isopropylidene- $\alpha$-D-xylofuranose (33)}

Obtained according to the general procedure, starting from 5-(4-aminomethyl-1H-1,2,3triazol-1-yl)-5-deoxy-3- $O$-dodecyl-1,2- $O$-isopropylidene- $\alpha$-D-xylofuranose $(\mathbf{3 0}, 89 \mathrm{mg}$, $0.2 \mathrm{mmol})$ and diethyl phosphorochloridate $(0.04 \mathrm{~mL}, 0.24 \mathrm{mmol})$. Purification by flash column chromatography (from EtOAc to EtOAc/MeOH, 28:1, then 22:1) afforded the title compound $(58 \mathrm{mg}, 50 \%)$ as a colourless oil.

$[\alpha]_{D}^{20}=-23\left(\mathrm{c}=1\right.$, in $\left.\mathrm{CH}_{2} \mathrm{Cl}_{2}\right) .{ }^{1} \mathrm{H} \mathrm{NMR}\left(400 \mathrm{MHz}, \mathrm{CDCl}_{3}\right): \delta 7.63(\mathrm{~s}, 1 \mathrm{H}, \mathrm{H}-5), 5.93$ $\left(\mathrm{d}, 1 \mathrm{H}, \mathrm{H}-1^{\prime}, J_{1^{\prime}, 2^{\prime}}=3.6\right), 4.70\left(\mathrm{dd}, 1 \mathrm{H}, \mathrm{H}-5^{\prime} \mathrm{a}, J_{4^{\prime}, 5^{\prime} \mathrm{a}}=8.3, J_{5^{\prime} \mathrm{a}, 5^{\prime} \mathrm{b}}=18.9\right), 4.58(\mathrm{~d}, 1 \mathrm{H}$, $\left.\mathrm{H}-2^{\prime}\right)$, 4.55-4.41 (m, $\left.2 \mathrm{H}, \mathrm{H}-4^{\prime}, \mathrm{H}-5^{\prime} \mathrm{b}\right), 4.21\left(\mathrm{dd}, 2 \mathrm{H}, \mathrm{CH}_{2} \mathrm{NH}, J_{\mathrm{CH}, \mathrm{P}}=10.7, J_{\mathrm{CH} 2, \mathrm{NH}}=\right.$ 7.0), 4.14-3.97 (m, $4 \mathrm{H}, 2 \times \mathrm{CH}_{2}, 2 \times \mathrm{Et}$ ), 3.85 (d, $1 \mathrm{H}, \mathrm{H}-3, J_{3^{\prime}, 4^{\prime}}=2.1$ ), 3.63 (dt, $1 \mathrm{H}$, $\left.\mathrm{H}-1^{\prime \prime} \mathrm{a}, J_{1^{\prime \prime}, 2^{\prime \prime} \mathrm{a}}=J_{1^{\prime \prime} \mathrm{a}, 2^{\prime \prime} \mathrm{b}}=6.5, J_{1 " \mathrm{a}, 1^{\prime \prime} \mathrm{b}}=9.1\right), 3.42\left(\mathrm{dt}, 1 \mathrm{H}, \mathrm{H}-1^{\prime \prime} \mathrm{b}, J_{1^{\prime \prime} \mathrm{b}, 2^{\prime \prime} \mathrm{a}}=J_{1^{\prime \prime} \mathrm{b}, 2^{\prime \prime} \mathrm{b}}=6.5\right)$, 
$3.18\left(\mathrm{dt}, 1 \mathrm{H}, \mathrm{N} H, J_{\mathrm{N} H, \mathrm{P}}=10.3\right), 1.61-1.50\left(\mathrm{~m}, 2 \mathrm{H}, \mathrm{CH}_{2}-2^{\prime \prime}\right), 1.41\left(\mathrm{~s}, 3 \mathrm{H}, \mathrm{CH}_{3}, i-\mathrm{Pr}\right)$, 1.37-1.15 (m, $27 \mathrm{H}, \mathrm{CH}_{3}, i-\mathrm{Pr}, 2 \times \mathrm{CH}_{3}, 2 \times \mathrm{Et}, \mathrm{CH}_{2}-3^{\prime \prime}$ to $\left.\mathrm{CH}_{2}-11^{\prime \prime}\right), 0.86$ (t, $3 \mathrm{H}, \mathrm{CH}_{3^{-}}$ $\left.12^{\prime \prime}, J=6.9\right) .{ }^{13} \mathrm{C}$ NMR (100 MHz, $\left.\mathrm{CDCl}_{3}\right): \delta 146.7\left(\mathrm{~d}, \mathrm{C}-4, J_{\mathrm{C}-4, \mathrm{P}}=6.2\right), 122.8(\mathrm{C}-5)$, 112.1 (Cq, $i$-Pr), $105.3\left(\mathrm{C}-1^{\prime}\right), 82.7\left(\mathrm{C}-3^{\prime}\right), 82.1\left(\mathrm{C}-2^{\prime}\right), 79.1\left(\mathrm{C}-4^{\prime}\right), 70.7\left(\mathrm{C}-1^{\prime \prime}\right), 62.6$ (d, $\left.2 \times \mathrm{CH}_{2}, 2 \times \mathrm{Et}, J_{\mathrm{C}, \mathrm{P}}=5.4\right), 49.6\left(\mathrm{C}^{-5}\right), 37.1\left(\mathrm{CH}_{2} \mathrm{~N}\right), 32.0,29.8,29.8,29.8,29.7,29.5$, 29.5, $26.3\left(\mathrm{CH}_{2}-3^{\prime \prime}\right.$ to $\left.\mathrm{CH}_{2}-10^{\prime \prime}\right)$ 26.9, $26.2\left(2 \times \mathrm{CH}_{3}, i\right.$-Pr), $22.8\left(\mathrm{CH}_{2}-10^{\prime \prime}\right), 16.3(\mathrm{~d}, 2 \times$ $\left.\mathrm{CH}_{3}, 2 \times \mathrm{Et}, J_{\mathrm{C}, \mathrm{P}}=7.1\right), 14.3\left(\mathrm{C}-12^{\prime \prime}\right) .{ }^{31} \mathrm{P} \mathrm{NMR}\left(162 \mathrm{MHz}, \mathrm{CDCl}_{3}\right): \delta=8.20 . \mathrm{HRMS}$ : calcd for $\mathrm{C}_{27} \mathrm{H}_{51} \mathrm{~N}_{4} \mathrm{O}_{7} \mathrm{P}[M+\mathrm{H}]^{+} 575.3568$, found 575.3572 ; calcd for $[M+\mathrm{Na}]^{+}$ 597.3388, found 597.3390.

\section{5-Deoxy-3-O-dodecyl-5-[5-(diethylphosphono)aminomethyl-1H-1,2,3-triazol-1-yl]- 1,2- $O$-isopropylidene- $\alpha$-D-xylofuranose (34)}

Obtained according to the general procedure, starting from 5-(5-aminomethyl-1H-1,2,3triazol-1-yl)-5-deoxy-3- $O$-dodecyl-1,2- $O$-isopropylidene- $\alpha$-D-xylofuranose $\quad(31, \quad 155$ $\mathrm{mg}, 0.35 \mathrm{mmol})$ and diethyl phosphorochloridate $(0.06 \mathrm{~mL}, 0.42 \mathrm{mmol})$. Purification by flash column chromatography (from EtOAc to EtOAc/MeOH, 28:1, then 22:1) afforded the title compound (105 $\mathrm{mg}, 52 \%)$ as a colourless oil.

$[\alpha]_{D}^{20}=-16\left(\mathrm{c}=1\right.$, in $\left.\mathrm{CH}_{2} \mathrm{Cl}_{2}\right) .{ }^{1} \mathrm{H} \mathrm{NMR}\left(400 \mathrm{MHz}, \mathrm{CDCl}_{3}\right): \delta 7.63(\mathrm{~s}, 1 \mathrm{H}, \mathrm{H}-4), 5.92$ $\left(\mathrm{d}, 1 \mathrm{H}, \mathrm{H}-1^{\prime}, J_{1^{\prime}, 2^{\prime}}=3.6\right), 4.73\left(\mathrm{dd}\right.$, part A of ABX system, $1 \mathrm{H}, \mathrm{H}-5^{\prime} \mathrm{a}, J_{4^{\prime}, 5^{\prime} \mathrm{a}}=2.8, J_{5^{\prime} \mathrm{a}, 5^{\prime} \mathrm{b}}$ =14.7), 4.62-4.50 (m, 2 H, H-2', H-4'), 4.40 (dd, part B of ABX system, $1 \mathrm{H}, \mathrm{H}-5$ 'b, $\left.J_{4^{\prime}, 5^{\prime} \mathrm{b}}=8.6\right), 4.29\left(\mathrm{dt}, 1 \mathrm{H}, \mathrm{H}-\mathrm{a}\right.$ from $\left.\mathrm{CH}_{2} \mathrm{NH}, J_{\mathrm{H}-\mathrm{a}, \mathrm{NH}}=J_{\mathrm{H}-\mathrm{a}, \mathrm{P}}=9.5, J_{\mathrm{a}, \mathrm{b}}=15.7\right), 4.17$ $\left(\mathrm{ddd}, 1 \mathrm{H}, \mathrm{H}-\mathrm{b}\right.$ from $\left.\mathrm{CH}_{2} \mathrm{NH}, J_{\mathrm{N} H, \mathrm{Hb}}=5.4, J_{\mathrm{H}-\mathrm{b}, \mathrm{P}}=12.1\right), 4.11-3.89\left(\mathrm{~m}, 4 \mathrm{H}, 2 \times \mathrm{CH}_{2}, 2\right.$ $\left.\times \mathrm{Et}, \mathrm{H}-3^{\prime}, J_{3^{\prime}, 4^{\prime}}=3.0\right), 3.64\left(\mathrm{dt}, 1 \mathrm{H}, \mathrm{H}-1^{\prime \prime} \mathrm{a}, J_{1^{\prime \prime} \mathrm{a}, 1^{\prime \prime} \mathrm{b}}=9.2, J_{1^{\prime \prime} \mathrm{a}, 2^{\prime \prime} \mathrm{a}}=J_{1^{\prime \prime} \mathrm{a}, 2^{\prime \prime} \mathrm{b}}=6.6\right), 3.57-$ $3.38\left(\mathrm{~m}, 2 \mathrm{H}, \mathrm{H}-1^{\prime \prime} \mathrm{b}, \mathrm{N} H, J_{1^{\prime \prime} \mathrm{b}, 2^{\prime \prime} \mathrm{a}}=J_{1^{\prime \prime} \mathrm{b}, 2^{\prime \prime} \mathrm{b}}=6.7, J_{\mathrm{N} H, \mathrm{P}}=J_{\mathrm{H}-\mathrm{a}, \mathrm{N} H}=9.5, J_{\mathrm{N} H, \mathrm{Hb}}=5.4\right), 1.63-$ $1.50\left(\mathrm{~m}, 2 \mathrm{H}, \mathrm{CH}_{2}-2^{\prime \prime}\right), 1.38$ (s, $\left.3 \mathrm{H}, \mathrm{CH}_{3}, i-\mathrm{Pr}\right), 1.35-1.19\left(\mathrm{~m}, 27 \mathrm{H}, \mathrm{CH}_{3}, i-\mathrm{Pr}, 2 \times \mathrm{CH}_{3}\right.$, $2 \times \mathrm{Et}, \mathrm{CH}_{2}-3^{\prime \prime}$ to $\left.\mathrm{CH}_{2}-11^{\prime \prime}\right), 0.86\left(\mathrm{t}, 3 \mathrm{H}, \mathrm{CH}_{3}-12^{\prime \prime}, J=6.9\right) .{ }^{13} \mathrm{C}$ NMR (100 MHz, $\left.\mathrm{CDCl}_{3}\right): \delta 136.5\left(\mathrm{~d}, \mathrm{C}-5, J_{\mathrm{C}-5, \mathrm{P}}=6.0\right), 133.5(\mathrm{C}-4), 112.3\left(\mathrm{Cq}, i\right.$-Prop), $105.2\left(\mathrm{C}-1^{\prime}\right), 82.9$ $\left(\mathrm{C}-3^{\prime}\right), 82.1\left(\mathrm{C}-2^{\prime}\right), 80.0\left(\mathrm{C}-4^{\prime}\right), 70.6\left(\mathrm{C}-1^{\prime \prime}\right), 62.8,62.7\left(2 \mathrm{~d}, 2 \times \mathrm{CH}_{2}, 2 \times \mathrm{Et}, J_{\mathrm{C}-\mathrm{P}}=5.2\right)$, $47.9\left(\mathrm{C}-5{ }^{\prime}\right), 34.2\left(\mathrm{CH}_{2} \mathrm{~N}\right), 32.0,29.8,29.7,29.7,29.5,29.5,26.3\left(\mathrm{CH}_{2}-3^{\prime \prime}\right.$ to $\left.\mathrm{CH}_{2}-10^{\prime \prime}\right)$ 26.8, $26.2\left(2 \times \mathrm{CH}_{3}, i\right.$-Pr), $22.8\left(\mathrm{CH}_{2}-11^{\prime \prime}\right), 16.30,16.25\left(\mathrm{~d}, 2 \times \mathrm{CH}_{3}, 2 \times \mathrm{Et}, J_{\mathrm{C}, \mathrm{P}}=6.8\right)$, $14.2\left(\mathrm{C}-12{ }^{\prime \prime}\right) .{ }^{31} \mathrm{P}$ NMR (162 MHz, $\left.\mathrm{CDCl}_{3}\right): \delta 7.83$. HRMS: calcd for $\mathrm{C}_{27} \mathrm{H}_{51} \mathrm{~N}_{4} \mathrm{O}_{7} \mathrm{P}[\mathrm{M}$ $+\mathrm{H}]^{+} 575.3568$, found 575.3570 ; calcd for $[\mathrm{M}+\mathrm{Na}]^{+} 597.3388$, found 597.3388.

\section{3-O-Benzyl-5-(4-bromomethyl-1H-1,2,3-triazol-1-yl)-5-deoxy-1,2-O-}

isopropylidene- $\alpha$-D-xylofuranose (35) and 3-O-benzyl-5-(5-bromomethyl-1H-1,2,3triazol-1-yl)-5-deoxy-1,2- $O$-isopropylidene- $\alpha$-D-xylofuranose (36)

Obtained according to the general procedure for thermal azide-alkyne cycloaddition, starting from 5-azido-3-O-benzyl-5-deoxy-1,2- $O$-isopropylidene- $\alpha$-D-xylofuranose (18, $159 \mathrm{mg}, 0.52 \mathrm{mmol}$ ) and propargyl bromide (80 wt.\% soln. in toluene, $0.12 \mathrm{~mL}, 1.04$ $\mathrm{mmol})$. The reaction was stirred for $16 \mathrm{~h}$. Purification by column chromatography (EtOAc/cyclohexane, 1:6) afforded the 1,4-disubstituted triazole 35 (106 mg, 48\%) as white crystals and the 1,5-disubstituted regioisomer $\mathbf{3 6}(4 \mathrm{mg}, 2 \%)$ as yellowish oil. 
Data for 35: $[\alpha]_{D}^{20}=-19\left(\mathrm{c}=1\right.$, in $\left.\mathrm{CH}_{2} \mathrm{Cl}_{2}\right)$. m.p.: $122.2-123.7^{\circ} \mathrm{C} .{ }^{1} \mathrm{H}$ NMR $(400$ $\left.\mathrm{MHz}, \mathrm{CDCl}_{3}\right): \delta 7.57$ (s, $\left.1 \mathrm{H}, \mathrm{H}-5\right), 7.42-7.30(\mathrm{~m}, 5 \mathrm{H}, \mathrm{Ph}), 5.97\left(\mathrm{~d}, 1 \mathrm{H}, \mathrm{H}-1^{\prime}, J_{1^{\prime}, 2^{\prime}}=\right.$ 3.7), 4.73 (d, part A of AB system, $1 \mathrm{H}, \mathrm{H}-\mathrm{a}$ from $\left.\mathrm{CH}_{2} \mathrm{Ph}, J_{\mathrm{a}, \mathrm{b}}=11.8\right), 4.70-4.59$ (m, 2 H, H-2', H-5'a, $J_{4^{\prime}, 5^{\prime} \mathrm{a}}=7.4, J_{5^{\prime} \mathrm{a}, 5^{\prime} \mathrm{b}}=17.1$ ), 4.57-4.42 (m, $4 \mathrm{H}, \mathrm{H}-4^{\prime}, \mathrm{H}-5{ }^{\prime} \mathrm{b}, \mathrm{CH}_{2} \mathrm{Br}, \mathrm{H}-\mathrm{b}$ from $\left.\mathrm{CH}_{2} \mathrm{Ph}\right), 4.50\left(\mathrm{~d}, 1 \mathrm{H}\right.$, Part B of $\mathrm{ABX}$ system, $\left.\mathrm{CH}_{2}-\mathrm{Bn}, J_{\mathrm{b}, \mathrm{a}}=11.9\right), 3.97(\mathrm{~d}, 1 \mathrm{H}$, $\mathrm{H}-3^{\prime}, J_{3^{\prime}, 4^{\prime}}=2.7$ ), 1.42 (s, $3 \mathrm{H}, \mathrm{CH}_{3}, i$-Pr), 1.30 (s, $3 H$, $\mathrm{CH}_{3}, i$-Pr). ${ }^{13} \mathrm{C} \mathrm{NMR}(100 \mathrm{MHz}$, $\left.\mathrm{CDCl}_{3}\right): \delta 144.6(\mathrm{C}-4), 136.8(\mathrm{Cq}, \mathrm{Ph}), 128.8,128.5,128.1(\mathrm{CH}, \mathrm{Ph}), 124.0(\mathrm{C}-5), 112.2$ (Cq, i-Prop), $105.3\left(\mathrm{C}-1 '^{\prime}\right), 82.0\left(\mathrm{C}-2^{\prime}\right), 81.5\left(\mathrm{C}-3^{\prime}\right), 78.8\left(\mathrm{C}-4^{\prime}\right), 72.0\left(\mathrm{CH}_{2} \mathrm{Ph}\right), 49.5(\mathrm{C}-$ 5'), 26.8, $26.3\left(2 \times \mathrm{CH}_{3}, i\right.$-Pr), $21.7\left(\mathrm{CH}_{2} \mathrm{Br}\right)$. HRMS: calcd for $\mathrm{C}_{18} \mathrm{H}_{22} \mathrm{BrN}_{3} \mathrm{O}_{4}[\mathrm{M}+\mathrm{H}]^{+}$ 424.0866, found 424.0867 .

Data for 36: $[\alpha]_{D}^{20}=-26\left(\mathrm{c}=1\right.$, in $\left.\mathrm{CH}_{2} \mathrm{Cl}_{2}\right) .{ }^{1} \mathrm{H} \mathrm{NMR}\left(400 \mathrm{MHz}, \mathrm{CDCl}_{3}\right): \delta 7.65(\mathrm{~s}, 1$ $\mathrm{H}, \mathrm{H}-4), 7.43-7.29(\mathrm{~m}, 5 \mathrm{H}, \mathrm{Ph}) 5.97\left(\mathrm{~d}, 1 \mathrm{H}, \mathrm{H}-1^{\prime}, J_{1^{\prime}, 2^{\prime}}=3.7\right), 4.75$ (d, part A of AB system, $1 \mathrm{H}, \mathrm{H}-\mathrm{a}$ from $\left.\mathrm{CH}_{2} \mathrm{Ph}, J_{\mathrm{a}, \mathrm{b}}=11.8\right), 4.71-4.56\left(\mathrm{H}-2^{\prime}, \mathrm{H}-4^{\prime}, \mathrm{CH}_{2}-5^{\prime}, \mathrm{H}-\mathrm{a}\right.$ from $\mathrm{CH}_{2} \mathrm{Br}$ ), 4.54-4.42 (m, $2 \mathrm{H}, \mathrm{H}-\mathrm{b}$ from $\mathrm{CH}_{2} \mathrm{Ph}, \mathrm{H}-\mathrm{b}$ from $\mathrm{CH}_{2} \mathrm{Br}, J_{\mathrm{a}, \mathrm{b}}=12.1$ ), 4.07 (d, 1 $\left.\mathrm{H}, \mathrm{H}-3^{\prime}, J_{3^{\prime}, 4^{\prime}}=2.6\right), 1.41\left(\mathrm{~s}, 3 \mathrm{H}, \mathrm{CH}_{3}, i\right.$-Pr), 1.30 ( s, $3 \mathrm{H}, \mathrm{CH}_{3}, i$-Pr). ${ }^{13} \mathrm{C} \mathrm{NMR}(100$ $\left.\mathrm{MHz}, \mathrm{CDCl}_{3}\right): \delta 136.0(\mathrm{C}-5), 134.2(\mathrm{Cq}, \mathrm{Ph}), 134.0(\mathrm{C}-4), 128.9,128.5,128.1(\mathrm{CH}$, $\mathrm{Ph}), 112.3\left(\mathrm{Cq}, i\right.$-Pr), $105.3\left(\mathrm{C}-1^{\prime}\right), 82.2\left(\mathrm{C}-2^{\prime}\right), 81.8\left(\mathrm{C}-3^{\prime}\right), 79.6\left(\mathrm{C}-4^{\prime}\right), 72.2\left(\mathrm{CH}_{2} \mathrm{Ph}\right)$, $47.8\left(\mathrm{C}-5^{\prime}\right), 26.8,26.3\left(2 \times \mathrm{CH}_{3}, i-\mathrm{Pr}\right), 17.5\left(\mathrm{CH}_{2} \mathrm{Br}\right)$.

\section{5-(4-Bromomethyl-1H-1,2,3-triazol-1-yl)-5-deoxy-3-O-dodecyl-1,2-O-}

\section{isopropylidene- $\alpha$-D-xylofuranose (37) and 5-(5-bromomethyl-1H-1,2,3-triazol-1-yl)-} 5-deoxy-3- $O$-dodecyl-1,2- $O$-isopropylidene- $\alpha$-D-xylofuranose (38)

Obtained according to the general procedure for thermal azide-alkyne cycloaddition, starting from 5-azido-5-deoxy-3- $O$-dodecyl-1,2- $O$-isopropylidene- $\alpha$-D-xylofuranose (19, $300 \mathrm{mg}, 0.78 \mathrm{mmol}$ ) and propargyl bromide (80 wt.\% soln. in toluene, $0.17 \mathrm{~mL}$, $1.56 \mathrm{mmol})$. The reaction was stirred for $48 \mathrm{~h}$. Purification by column chromatography (EtOAc/cyclohexane, 1:7) afforded the 1,4-disubstituted triazole 35 (227 mg, 58\%) as and the 1,5-disubstituted regioisomer $\mathbf{3 6}(20 \mathrm{mg}, 5 \%)$ as yellowish oils.

Data for 37: $[\alpha]_{D}^{20}=-12\left(\mathrm{c}=1\right.$, in $\left.\mathrm{CH}_{2} \mathrm{Cl}_{2}\right) .{ }^{1} \mathrm{H} \mathrm{NMR}\left(400 \mathrm{MHz}, \mathrm{CDCl}_{3}\right): \delta 7.71(\mathrm{~s}, 1$ H, H-5), 5.95 (d, $\left.1 \mathrm{H}, \mathrm{H}-1^{\prime}, J_{1^{\prime}, 2^{\prime}}=3.7\right), 4.71$ (m, $\left.1 \mathrm{H}, \mathrm{H}-5^{\prime} \mathrm{a}, J_{4^{\prime}, 5^{\prime} \mathrm{a}}=2.2, J_{5^{\prime} \mathrm{a}, 5^{\prime} \mathrm{b}}=12.3\right)$, 4.63-4.43 (m, 5 H, H-2', H-4', H-5'b, CH $\mathrm{CH}_{2} \mathrm{Br}$ ), 3.84 (d, 1H, H-3', $J_{3^{\prime}, 4^{\prime}}=2.7$ ), 3.64 (dt, 1 $\left.\mathrm{H}, \mathrm{H}-1{ }^{\prime \prime} \mathrm{a}, J_{1 " \mathrm{a}, 2^{\prime \prime} \mathrm{a}}=J_{1 " \mathrm{a}, 2^{\prime \prime} \mathrm{b}}=6.5, J_{1 " \mathrm{a}, 1^{\prime \prime} \mathrm{b}}=9.2\right), 3.41\left(\mathrm{dt}, 1 \mathrm{H}, \mathrm{H}-1^{\prime \prime} \mathrm{b}, J_{1^{\prime \prime} \mathrm{b}, 2^{\prime \prime} \mathrm{a}}=J_{1^{\prime \prime} \mathrm{b}, 2^{\prime \prime} \mathrm{b}}=\right.$ 6.7), 1.62-1.50 (m, $\left.2 \mathrm{H}, \mathrm{CH}_{2}-2^{\prime \prime}\right), 1.43$ (s, $3 \mathrm{H}, \mathrm{CH}_{3}, i$-Pr), 1.39-1.16 (m, $21 \mathrm{H}, \mathrm{CH}_{3}, i$ Pr, $\mathrm{CH}_{2}-3$ "to $\left.\mathrm{CH}_{2}-11^{\prime \prime}\right), 0.87$ (t, $3 \mathrm{H}, \mathrm{CH}_{3}-12$ ", $\left.J=6.9\right) .{ }^{13} \mathrm{C} \mathrm{NMR}\left(100 \mathrm{MHz}, \mathrm{CDCl}_{3}\right): \delta$ 144.7 (C-4), 124.1 (C-5), $112.2(\mathrm{Cq}, i-\mathrm{Pr}), 105.4\left(\mathrm{C}-1^{\prime}\right), 82.6\left(\mathrm{C}-3^{\prime}\right), 82.1\left(\mathrm{C}-2^{\prime}\right), 78.9$ (C-4'), 70.7 (C-1"'), 49.6 (C-5'), 32.1, 29.8, 29.8, 29.7, 29.5, 29.5, 26.3 (C-2" to C-10"), 26.9, $26.2\left(2 \times \mathrm{CH}_{3}, i\right.$-Pr $), 22.8\left(\mathrm{C}-11^{\prime \prime}\right), 21.8\left(\mathrm{CH}_{2} \mathrm{Br}\right), 14.3\left(\mathrm{C}-12^{\prime \prime}\right)$. HRMS: calcd for $\mathrm{C}_{23} \mathrm{H}_{40} \mathrm{BrN}_{3} \mathrm{O}_{4}[M+\mathrm{H}]^{+}$502.2275, found 502.2277. 
Data for 38: $[\alpha]_{D}^{20}=-50\left(\mathrm{c}=1\right.$, in $\left.\mathrm{CH}_{2} \mathrm{Cl}_{2}\right) .{ }^{1} \mathrm{H} \mathrm{NMR}\left(400 \mathrm{MHz}, \mathrm{CDCl}_{3}\right): \delta 7.67(\mathrm{~s}, 1$ $\mathrm{H}, \mathrm{H}-4), 5.93$ (d, 1H, H-1', $J_{1^{\prime}, 2^{\prime}}=3.7$ ), 4.80-4.67 (m, $2 \mathrm{H}, \mathrm{H}-5^{\prime} \mathrm{a}, \mathrm{H}-\mathrm{a}$ from $\mathrm{CH}_{2} \mathrm{Br}$ ), 4.65-4.47 (m, 4 H, H-2', H-4', H-5'b, H-b from $\mathrm{CH}_{2} \mathrm{Br}$ ), 3.94 (br. s, 1H, H-3'), 3.66 (dt, $\left.1 \mathrm{H}, \mathrm{H}-1^{\prime \prime} \mathrm{a}, J_{1 " \mathrm{a}, 2^{\prime \prime} \mathrm{a}}=J_{1^{\prime \prime} \mathrm{a}, 2^{\prime \prime} \mathrm{b}}=6.5, J_{1 " \mathrm{a}, 1^{\prime \prime} \mathrm{b}}=9.1\right), 3.44\left(\mathrm{dt}, 1 \mathrm{H}, \mathrm{H}-1^{\prime \prime} \mathrm{b}, J_{1 " \mathrm{~b}, 2^{\prime \prime} \mathrm{a}}=J_{1^{\prime \prime} \mathrm{b}, 2^{\prime \prime} \mathrm{b}}=\right.$ 6.6), 1.67-1.49 (m, $\left.2 \mathrm{H}, \mathrm{CH}_{2}-2^{\prime \prime}\right), 1.42$ (s, $3 \mathrm{H}, \mathrm{CH}_{3}, i$-Pr), 1.37-1.19 (m, $21 \mathrm{H}, \mathrm{CH}_{3}, i$ Pr, $\mathrm{CH}_{2}-3^{\prime \prime}$ to $\left.\mathrm{CH}_{2}-11^{\prime \prime}\right), 0.87$ (t, $\left.3 \mathrm{H}, \mathrm{CH}_{3}-12^{\prime \prime}, J=6.9\right) .{ }^{13} \mathrm{C} \mathrm{NMR}\left(100 \mathrm{MHz}, \mathrm{CDCl}_{3}\right): \delta$ $=133.4(\mathrm{C}-4)^{*}, 112.2(\mathrm{Cq}, i-\mathrm{Pr}), 105.3\left(\mathrm{C}-1^{\prime}\right), 82.7\left(\mathrm{C}-3^{\prime}\right), 82.2\left(\mathrm{C}-2^{\prime}\right), 79.7\left(\mathrm{C}-4^{\prime}\right), 70.7$ $\left(\mathrm{C}-1^{\prime \prime}\right), 47.8$ (C-5'), 32.0, 29.8, 29.8, 29.7, 29.7, 29.5, 29.5, 27.0, 26.3 (C-2" to C-10"), 26.8, $26.2\left(2 \times \mathrm{CH}_{3}, i\right.$-Pr), $22.8\left(\mathrm{C}-11^{\prime \prime}\right), 17.5\left(\mathrm{CH}_{2} \mathrm{Br}\right), 14.3\left(\mathrm{C}-12^{\prime \prime}\right)$. *inferred from HSQC. HRMS: calcd for $\mathrm{C}_{23} \mathrm{H}_{40} \mathrm{BrN}_{3} \mathrm{O}_{4}[M+\mathrm{H}]^{+} 502.2275$, found 502.2276.

General procedure for the Michaelis-Arbuzov reaction between 3-O-protected 5(bromomethyl-1H-1,2,3-triazol-1-yl)-1,2-O-isopropylidene- $\alpha$-D-xylofuranoses with triethyl phosphite.

A solution of 5-(bromomethyl-1H-1,2,3-triazol-1-yl) xylofuranose derivative $(0.17$ mmol) in triethyl phosphite $(1.5 \mathrm{~mL})$ was stirred at $110^{\circ} \mathrm{C}$ for $2 \mathrm{~h}$. The solution was concentrated under vacuum and the residue was subjected to column chromatography.

\section{3-O-Benzyl-5-deoxy-5-[4-(diethylphosphono)methyl-1H-1,2,3-triazol-1-yl]-1,2-O- isopropylidene- $\alpha$-D-xylofuranose (39)}

Obtained according to the general procedure, starting from 3-O-benzyl-5-(4bromomethyl-1 $H$-1,2,3-triazol-1-yl)-5-deoxy-1,2- $O$-isopropylidene- $\alpha$-D-xylofuranose (35, $80 \mathrm{mg}, 0.19 \mathrm{mmol})$ and triethyl phosphite $(1.5 \mathrm{~mL})$. Purification by column chromatography (EtOAc/hexane, 1:3) afforded the title compound (75 $\mathrm{mg}, 83 \%)$ as a colourless oil.

$[\alpha]_{D}^{20}=-33\left(\mathrm{c}=1\right.$, in $\left.\mathrm{CH}_{2} \mathrm{Cl}_{2}\right) .{ }^{1} \mathrm{H}$ NMR $\left(400 \mathrm{MHz}, \mathrm{CDCl}_{3}\right): 7.64(\mathrm{~d}, 1 \mathrm{H}, \mathrm{H}-5, J=$ 2.1), 7.43-7.29 (m, $5 \mathrm{H}, \mathrm{Ph}), 5.98\left(\mathrm{~d}, 1 \mathrm{H}, \mathrm{H}^{-1} 1^{\prime}, J_{1^{\prime}, 2^{\prime}}=3.7\right), 4.72(\mathrm{~d}, 1 \mathrm{H}$, part A of AB system, H-a, $\left.\mathrm{CH}_{2} \mathrm{Ph}, J_{\mathrm{a}, \mathrm{b}}=11.6\right)$, 4.68-4.60 (m, $2 \mathrm{H}, \mathrm{H}-2^{\prime}, \mathrm{H}-5^{\prime} \mathrm{a}, J_{5^{\prime} \mathrm{a}, 5^{\prime} \mathrm{b}}=13.3, J_{4^{\prime}, 5^{\prime} \mathrm{a}}=$ 7.7), 4.57-4.46 (m, $3 \mathrm{H}, \mathrm{H}-4^{\prime}, \mathrm{H}-5$ 'b, H-b from $\mathrm{CH}_{2} \mathrm{Ph}$ ), 4.14-4.01 (m, $4 \mathrm{H}, 2 \times \mathrm{CH}_{2}, 2 \times$ Et), $3.97\left(\mathrm{~d}, 1 \mathrm{H}, \mathrm{H}-3^{\prime}, J_{3^{\prime}, 4^{\prime}}=2.5\right), 3.38-3.21\left(\mathrm{~m}, 2 \mathrm{H}, \mathrm{CH}_{2} \mathrm{P}, J_{\mathrm{a}, \mathrm{b}}=20.5, J_{\mathrm{H}-\mathrm{a}, \mathrm{P}}=15.8, J_{\mathrm{H}-}\right.$ b,P $=16.0), 1.41\left(\mathrm{~s}, 3 \mathrm{H}, \mathrm{CH}_{3}, i-\mathrm{Pr}\right), 1.33-1.21\left(\mathrm{~m}, 9 \mathrm{H}, \mathrm{CH}_{3}, i-\mathrm{Pr}, 2 \times \mathrm{CH}_{3}, 2 \times \mathrm{Et}\right) .{ }^{13} \mathrm{C}$ NMR (100 MHz, $\left.\mathrm{CDCl}_{3}\right): \delta 138.5\left(\mathrm{~d}, \mathrm{C}-4, J_{\mathrm{C}-4, \mathrm{P}}=7.1\right), 136.9(\mathrm{Cq}, \mathrm{Ph}), 128.8,128.4$, $128.0(\mathrm{CH}, \mathrm{Ph}), 123.9$ (d, C-5, $\left.J_{\mathrm{C}-5, \mathrm{P}}=4.1\right), 112.2\left(\mathrm{Cq}, i\right.$-Pr), $105.3\left(\mathrm{C}-1^{\prime}\right), 82.0\left(\mathrm{C}-2^{\prime}\right)$, $81.6\left(\mathrm{C}-3^{\prime}\right), 78.9\left(\mathrm{C}-4^{\prime}\right), 71.9\left(\mathrm{CH}_{2} \mathrm{Ph}\right), 62.49,62.45\left(2 \mathrm{~d}, 2 \times \mathrm{CH}_{2}, 2 \times \mathrm{Et}, J_{\mathrm{C}, \mathrm{P}}=6.5\right)$, $49.5\left(\mathrm{C}-5^{\prime}\right), 26.8,26.3\left(2 \times \mathrm{CH}_{3}, i\right.$-Pr), $24.3\left(\mathrm{~d}, \mathrm{CH}_{2} \mathrm{P}, J_{\mathrm{C}, \mathrm{P}}=142.6\right), 16.5\left(\mathrm{~d}, 2 \times \mathrm{CH}_{3}, 2\right.$ $\times$ Et, $\left.\quad J_{\mathrm{C}, \mathrm{P}}=6.0\right) .{ }^{31} \mathrm{P} \mathrm{NMR}\left(162 \mathrm{MHz}, \mathrm{CDCl}_{3}\right): \delta$ 24.87. HRMS: calcd for $\mathrm{C}_{22} \mathrm{H}_{32} \mathrm{~N}_{3} \mathrm{O}_{7} \mathrm{P}[\mathrm{M}+\mathrm{H}]^{+} 482.2051$, found 482.2063; calcd for $\mathrm{C}_{22} \mathrm{H}_{32} \mathrm{~N}_{3} \mathrm{O}_{7} \mathrm{P}[\mathrm{M}+\mathrm{Na}]^{+}$ 504.1870, found 504.1873. 


\section{5-Deoxy-5-[4-(diethylphosphono)methyl-1H-1,2,3-triazol-1-yl]-3-O-dodecyl-1,2-O- isopropylidene- $\alpha$-D-xylofuranose (40)}

Obtained according to the general procedure, starting from 5-(4-bromomethyl-1H-1,2,3triazol-1-yl)-5-deoxy-3-O-dodecyl-1,2- $O$-isopropylidene- $\alpha$-D-xylofuranose $(37,85 \mathrm{mg}$, $0.17 \mathrm{mmol})$ and triethyl phosphite $(1.5 \mathrm{~mL})$. Purification by column chromatography (EtOAc/hexane, 1:3) afforded the title compound (91 mg, 96\%) as a colourless oil.

$[\alpha]_{D}^{20}=-14\left(\mathrm{c}=1\right.$, in $\left.\mathrm{CH}_{2} \mathrm{Cl}_{2}\right) .{ }^{1} \mathrm{H} \mathrm{NMR}\left(400 \mathrm{MHz}, \mathrm{CDCl}_{3}\right): \delta 7.71(\mathrm{~d}, 1 \mathrm{H}, \mathrm{H}-5, J=$ 1.6), $5.94\left(\mathrm{~d}, 1 \mathrm{H}, \mathrm{H}-1^{\prime}, J_{1^{\prime}, 2^{\prime}}=3.6\right), 4.71\left(\mathrm{dd}, 1 \mathrm{H}\right.$, part A of ABX system, H-5'a, $J_{4^{\prime}, 5^{\prime} \mathrm{a}}=$ 7.9, $\left.J_{5^{\prime} \mathrm{a}, 5^{\prime} \mathrm{b}}=17.5\right), 4.59$ (d, $\left.1 \mathrm{H}, \mathrm{H}-2^{\prime}\right), 4.56-4.46$ (m, 2 H, H-4', H-5'b, ), 4.17-4.03 (m, 4 $\left.\mathrm{H}, 2 \times \mathrm{CH}_{2}, 2 \times \mathrm{Et}\right), 3.84\left(\mathrm{~d}, 1 \mathrm{H}, \mathrm{H}-3^{\prime}, J_{3^{\prime}, 4^{\prime}}=2.5\right), 3.64\left(\mathrm{dt}, 1 \mathrm{H}, \mathrm{H}-1^{\prime \prime} \mathrm{a}, J_{1^{\prime \prime} \mathrm{a}, 2^{\prime \prime} \mathrm{a}}=J_{1^{\prime \prime} \mathrm{a}, 2^{\prime \prime b}}\right.$ $\left.=6.6, J_{1 " \mathrm{a}, 1 " \mathrm{~b}}=9.0\right), 3.50-3.23\left(\mathrm{~m}, 3 \mathrm{H}, \mathrm{H}-1^{\prime \prime} \mathrm{b}, \mathrm{C} H_{2} \mathrm{P}, J_{1^{\prime \prime} \mathrm{b}, 2^{\prime \prime} \mathrm{a}}=J_{1^{\prime \prime} \mathrm{b}, 2^{\prime \prime} \mathrm{b}}=6.6, J_{\mathrm{a}, \mathrm{b}}(\mathrm{CH} 2 \mathrm{P})=\right.$ $\left.20.5, J_{\mathrm{H}-\mathrm{a}, \mathrm{P}}=15.7, J_{\mathrm{H}-\mathrm{b}, \mathrm{P}}=15.9\right), 1.66-1.52\left(\mathrm{~m}, 2 \mathrm{H}, \mathrm{CH}_{2}-2^{\prime \prime}\right), 1.43\left(\mathrm{~s}, 3 \mathrm{H}, \mathrm{CH}_{3}, i-\mathrm{Pr}\right)$, 1.39-1.17 (m, $27 \mathrm{H}, \mathrm{CH}_{3}, i-\mathrm{Pr}, \mathrm{CH}_{2}-3^{\prime \prime}$ to $\left.\mathrm{CH}_{2}-11^{\prime \prime}, 2 \times \mathrm{CH}_{3}, 2 \times \mathrm{Et}\right), 0.88$ (t, $3 \mathrm{H}, \mathrm{CH}_{3}-$ $12 ", J=6.9) .{ }^{13} \mathrm{C}$ NMR (100 MHz, $\left.\mathrm{CDCl}_{3}\right): \delta=138.48\left(\mathrm{~d}, \mathrm{C}-4, J_{\mathrm{C}-4, \mathrm{P}}=7.2\right), 123.9$ (d, $\left.\mathrm{C}-5, J_{\mathrm{C}-5, \mathrm{P}}=3.4\right), 112.1(\mathrm{Cq}, i-\mathrm{Pr}), 105.3\left(\mathrm{C}-1^{\prime}\right), 82.5\left(\mathrm{C}-3^{\prime}\right), 82.1\left(\mathrm{C}-2^{\prime}\right), 79.0\left(\mathrm{C}-4^{\prime}\right), 70.6$ $\left(\mathrm{C}-1^{\prime \prime}\right), 62.53,62.51\left(2 \mathrm{~d}, 2 \times \mathrm{CH}_{2}, 2 \times \mathrm{Et}, J_{\mathrm{C}, \mathrm{P}}=6.6\right), 49.4\left(\mathrm{C}-5^{\prime}\right), 32.0,29.8,29.8$, 29.7, 29.7, 29.5, 29.5, 26.3 (C-2" to C-10"), 26.9, $26.2\left(2 \times \mathrm{CH}_{3}, i-\mathrm{Pr}\right), 24.3\left(\mathrm{~d}, \mathrm{CH}_{2} \mathrm{P}\right.$, $\left.J_{\mathrm{C}, \mathrm{P}}=142.6\right), 22.8\left(\mathrm{C}-11^{\prime \prime}\right), 16.5\left(\mathrm{~d}, 2 \times \mathrm{CH}_{3}, 2 \times \mathrm{Et}, J_{\mathrm{C}, \mathrm{P}}=6.0\right), 14.3\left(\mathrm{C}-12^{\prime \prime}\right) .{ }^{31} \mathrm{P} \mathrm{NMR}$ $\left(162 \mathrm{MHz}, \mathrm{CDCl}_{3}\right): \delta 24.91$ ppm. HRMS: calcd for $\mathrm{C}_{27} \mathrm{H}_{50} \mathrm{~N}_{3} \mathrm{O}_{7} \mathrm{P}[M+\mathrm{H}]^{+} 560.3459$, found 560.3462 .

\section{5-Deoxy-5-[5-(diethylphosphono)methyl-1H-1,2,3-triazol-1-yl]-3-O-dodecyl-1,2-O- isopropylidene- $\alpha$-D-xylofuranose (41)}

Obtained according to the general procedure, starting from 5-(5-bromomethyl-1H-1,2,3triazol-1-yl)-5-deoxy-3- $O$-dodecyl-1,2- $O$-isopropylidene- $\alpha$-D-xylofuranose $(38,90 \mathrm{mg}$, $0.18 \mathrm{mmol})$ and triethyl phosphite $(1.5 \mathrm{~mL})$. Purification by column chromatography (EtOAc/hexane, 1:3) afforded the title compound (76 mg, 76\%) as a colourless oil. $[\alpha]_{D}^{20}=-18\left(\mathrm{c}=1\right.$, in $\left.\mathrm{CH}_{2} \mathrm{Cl}_{2}\right) .{ }^{1} \mathrm{H} \mathrm{NMR}\left(400 \mathrm{MHz}, \mathrm{CDCl}_{3}\right): \delta 7.66$ (br.s, $\left.1 \mathrm{H}, \mathrm{H}-4\right)$, $5.90\left(\mathrm{~d}, 1 \mathrm{H}, \mathrm{H}-1^{\prime}, J_{1^{\prime}, 2^{\prime}}=3.6\right), 4.67$ (dd, part A of ABX system, $1 \mathrm{H}, \mathrm{H}-5^{\prime} \mathrm{a}, J_{4^{\prime} 5^{\prime} \mathrm{a}}=7.0$, $\left.J_{5^{\prime} \mathrm{a}, 5^{\prime} \mathrm{b}}=17.8\right), 4.61-4.46\left(\mathrm{~m}, 3 \mathrm{H}, \mathrm{H}-2^{\prime}, \mathrm{H}-4^{\prime}, \mathrm{H}-5^{\prime} \mathrm{b}\right), 4.16-3.99\left(\mathrm{~m}, 4 \mathrm{H}, 2 \times \mathrm{CH}_{2}, 2 \times\right.$ Et), $3.88\left(\mathrm{~d}, 1 \mathrm{H}, \mathrm{H}-3^{\prime}, J_{3^{\prime}, 4^{\prime}}=2.2\right), 3.61\left(\mathrm{dt}, 1 \mathrm{H}\right.$, part A of ABX system, H-1"a, $J_{1^{\prime \prime} \mathrm{a}, 2^{\prime \prime} \mathrm{a}}=$ $\left.J_{1^{\prime \prime}, 2^{\prime \prime} \mathrm{b}}=6.7, J_{1^{\prime \prime} \mathrm{a}, 1^{\prime \prime} \mathrm{b}}=9.0\right), 3.54-3.36\left(\mathrm{~m}, 2 \mathrm{H}, \mathrm{H}-1^{\prime \prime} \mathrm{b}, \mathrm{H}-\mathrm{a}\right.$ from $\mathrm{CH}_{2} \mathrm{P}, J_{1^{\prime \prime} \mathrm{b}, 2^{\prime \prime} \mathrm{a}}=J_{1^{\prime \prime} \mathrm{b}, 2^{\prime \prime} \mathrm{b}}=$ 6.6, $\left.J_{\mathrm{H}-\mathrm{a}, \mathrm{P}}=16.3\right), 3.29\left(\mathrm{dd}, \mathrm{H}-\mathrm{b}\right.$ from $\left.\mathrm{CH}_{2} \mathrm{P}, J_{\mathrm{a}, \mathrm{b}(\mathrm{CH} 2 \mathrm{P})}=21.2, J_{\mathrm{H}-\mathrm{b}, \mathrm{P}}=16.3\right), 1.63-1.48$ (m, $\left.2 \mathrm{H}, \mathrm{CH}_{2}-2^{\prime \prime}\right), 1.44-1.16\left(\mathrm{~m}, 30 \mathrm{H}, 2 \times \mathrm{CH}_{3}, i-\mathrm{Pr}, \mathrm{CH}_{2}-3\right.$ " to $\mathrm{CH}_{2}-11^{\prime \prime}, 2 \times \mathrm{CH}_{3}, 2 \times$ $\mathrm{Et}), 0.88$ (t, $\left.3 \mathrm{H}, \mathrm{CH}_{3}-12{ }^{\prime \prime}, J=6.9\right) .{ }^{13} \mathrm{C} \mathrm{NMR}\left(100 \mathrm{MHz}, \mathrm{CDCl}_{3}\right): \delta 134.0\left(\mathrm{~d}, \mathrm{C}-4, J_{\mathrm{C}-4, \mathrm{P}}\right.$ $=4.3), 129.4\left(\mathrm{~d}, \mathrm{C}-5, J_{\mathrm{C}-5, \mathrm{P}}=7.4\right), 112.1(\mathrm{Cq}, i-\mathrm{Pr}), 105.2\left(\mathrm{C}-1^{\prime}\right), 82.8\left(\mathrm{C}-3^{\prime}\right), 82.1(\mathrm{C}-$ $\left.2^{\prime}\right), 80.1\left(\mathrm{C}-4^{\prime}\right), 70.6\left(\mathrm{C}-1^{\prime \prime}\right), 62.76,62.73\left(2 \mathrm{~d}, 2 \times \mathrm{CH}_{2}, 2 \times \mathrm{Et}, J_{\mathrm{C}, \mathrm{P}}=6.5\right), 47.5\left(\mathrm{C}-5^{\prime}\right)$, 32.0, 29.7, 29.7, 29.5, 29.5, $26.3\left(\mathrm{C}-2^{\prime \prime}\right.$ to $\left.\mathrm{C}-10^{\prime \prime}\right), 26.8,26.2\left(2 \times \mathrm{CH}_{3}, i\right.$-Pr $), 22.8(\mathrm{C}-$ $\left.11^{\prime \prime}\right), 21.9\left(\mathrm{~d}, \mathrm{CH}_{2} \mathrm{P}, J_{\mathrm{C}, \mathrm{P}}=144.1\right), 16.5\left(\mathrm{~d}, 2 \times \mathrm{CH}_{3}, 2 \times \mathrm{Et}, J_{\mathrm{C}, \mathrm{P}}=6.0\right), 14.2\left(\mathrm{C}-12^{\prime \prime}\right) .{ }^{31} \mathrm{P}$ 
NMR (162 MHz, $\left.\mathrm{CDCl}_{3}\right): \delta$ 22.21. HRMS: calcd for $\mathrm{C}_{27} \mathrm{H}_{50} \mathrm{~N}_{3} \mathrm{O}_{7} \mathrm{P}[M+\mathrm{H}]^{+} 560.3459$, found 560.3479; calcd for $\mathrm{C}_{27} \mathrm{H}_{50} \mathrm{~N}_{3} \mathrm{O}_{7} \mathrm{P}[M+\mathrm{Na}]^{+}$582.3279, found 582.3295.

General procedure for TFA-mediated acetonide hydrolysis in 1,2-O-isopropylidene xylofuranose 5-(triazolyl)methyl phosphonates followed by acetylation

A solution of 3-O-protected 1,2- $O$-isopropylidene xylofuranose 5-(triazolyl)methyl phosphonate $(0.1 \mathrm{mmol})$ in aqueous trifluoroacetic acid $(1.5 \mathrm{~mL})$ was stirred at room temperature for $2 \mathrm{~h}$. The solvents were co-evaporated with toluene. The residue was dried under vacuum and was then treated with pyridine $(2 \mathrm{~mL})$ and acetic anhydride $(1.5 \mathrm{~mL})$. The mixture was stirred at room temp. for $90 \mathrm{~min}$. The solvents were coevaporated with toluene and the residue was subjected to column chromatography.

\section{1,2-Di- $O$-acetyl-3- $O$-benzyl-5-deoxy-5-[4-(diethylphosphono)methyl-1H-1,2,3- triazol-1-yl]- $\alpha, \beta$-D-xylofuranose $(42-\alpha, \beta)$}

Obtained according to the general procedure starting from 3-O-benzyl-5-deoxy-5-[4(diethylphosphono)methyl-1 $H$-1,2,3-triazol-1-yl]-1,2- $O$-isopropylidene- $\alpha$-D-

xylofuranose (39, $50 \mathrm{mg}, 0.1 \mathrm{mmol})$. Purification by column chromatography (EtOAc/hexane, 6:1) afforded the title compound $(28 \mathrm{mg}, 51 \%$, two steps, anomeric mixture, $\alpha / \beta$ ratio, $1: 0.5)$ as a colourless oil.

${ }^{1} \mathrm{H}$ NMR (400 MHz, $\mathrm{CDCl}_{3}$ ): $\delta$ 7.63, $7.62\left(2 \mathrm{~d}, 1.5 \mathrm{H}, \mathrm{H}-5^{\prime} \alpha, \mathrm{H}-5^{\prime} \beta\right)$ ) 7.42-7.29 (m, 7.5 $\mathrm{H}, \mathrm{Ph}), 6.44\left(\mathrm{~d}, 1 \mathrm{H}, \mathrm{H}-1^{\prime} \alpha, J_{1^{\prime}, 2^{\prime}(\alpha)}=4.5\right), 6.15$ (s, 0.5 H, H-1' $\beta$ ), 5.32-5.27 (m, 1.5 H, H$\left.2^{\prime} \alpha, \mathrm{H}-2^{\prime} \beta\right), 4.82\left(\mathrm{~d}, 0.5 \mathrm{H}\right.$, part $\mathrm{A}$ of $\mathrm{AB}$ system, $\mathrm{H}$-a from $\mathrm{CH}_{2} \mathrm{Ph}, \beta, J_{\mathrm{a}, \mathrm{b}(\beta)}=11.8$ ), 4.75-4.63 (m, 4 H, H-a from $\left.\mathrm{CH}_{2} \mathrm{Ph}, \alpha, \mathrm{H}^{-} 5^{\prime} \mathrm{a} \alpha, \mathrm{H}^{\prime} 5^{\prime} \mathrm{a} \beta, \mathrm{H}^{-} 4^{\prime} \alpha, \mathrm{H}^{\prime} 4^{\prime} \beta\right)$ ), 4.61-4.51 (m, 2 $\mathrm{H}, \mathrm{H}-\mathrm{b}$ from $\mathrm{CH}_{2} \mathrm{Ph}, \alpha, \mathrm{H}-\mathrm{b}$ from $\left.\mathrm{CH}_{2} \mathrm{Ph}, \beta, \mathrm{H}-5^{\prime} \mathrm{b} \beta\right), 4.46(\mathrm{dd}, 1 \mathrm{H}$, part B of ABX system, H-5'b $\left.\alpha, J_{4^{\prime}, 5^{\prime} \mathrm{b}(\alpha)}=7.7, J_{5^{\prime} \mathrm{a}, 5^{\prime} \mathrm{b}(\alpha)}=13.8\right), 4.25\left(\mathrm{~m}, 1 \mathrm{H}, \mathrm{H}-3^{\prime} \alpha, J_{2^{\prime}, 3^{\prime}(\alpha)}=3.7, J_{3^{\prime}, 4^{\prime}(\alpha)}\right.$ = 5.1), 4.15-4.01 (m, 6.5 H, H-3' $\left.\beta, 2 \times \mathrm{CH}_{2}, 2 \times \mathrm{Et}, \alpha, \beta\right), 3.38-3.21\left(\mathrm{~m}, 3 \mathrm{H}, \mathrm{CH}_{2} \mathrm{P}, \alpha, \beta\right.$, $\left.J_{\mathrm{a}, \mathrm{b}(\mathrm{CH} 2 \mathrm{P})}=18.0, J_{\mathrm{H}-\mathrm{a}, \mathrm{P}}=16.3, J_{\mathrm{H}-\mathrm{b}, \mathrm{P}}=15.8\right), 2.13,2.10\left(2 \mathrm{~s}, 3 \mathrm{H}, \mathrm{CH}_{3}, 2 \times \mathrm{Ac}, \beta\right), 2.07$, $2.03\left(2 \mathrm{~s}, 6 \mathrm{H}, \mathrm{CH}_{3}, 2 \times \mathrm{Ac}, \alpha\right), 1.28\left(\mathrm{t}, 9 \mathrm{H}, 2 \times \mathrm{CH}_{3}, 2 \times \mathrm{Et}, \alpha, \beta, J=7.0\right) .{ }^{13} \mathrm{C} \mathrm{NMR}$ $\left(100 \mathrm{MHz}, \mathrm{CDCl}_{3}\right): \delta 169.8,169.7,169.6,169.3(\mathrm{CO}, \mathrm{Ac}, \alpha, \beta), 138.6\left(\mathrm{~d}, \mathrm{C}-4, \alpha, \beta, J_{\mathrm{C}-}\right.$ $\left.{ }_{4, \mathrm{P}}=6.9\right), 137.0,136.8(\mathrm{Cq}, \mathrm{Ph}, \alpha, \beta), 128.8,128.8,128.5,128.4,128.1,128.1(\mathrm{CH}, \mathrm{Ph}$, $\alpha, \beta), 124.1\left(\mathrm{C}-5 \alpha, J_{\mathrm{C}-5, \mathrm{P}(\alpha)}=3.9\right), 124.0\left(\mathrm{C}-5 \beta, J_{\mathrm{C}-5, \mathrm{P}(\beta)}=4.3\right), 99.7\left(\mathrm{C}-1^{\prime} \beta\right), 94.1\left(\mathrm{C}-1^{\prime}\right.$ $\alpha), 81.7$ (C-4' $\beta$ ), 80.0, 80.0 (C-3' $\left.\alpha, \mathrm{C}-3^{\prime} \beta\right), 79.1$ (C- 2' $\beta$ ), $78.2\left(\mathrm{C}-4^{\prime} \alpha\right), 76.5\left(\mathrm{C}-2^{\prime} \alpha\right)$, $72.5,72.2\left(\mathrm{CH}_{2} \mathrm{Ph}, \alpha, \beta\right), 62.52,62.50\left(2 \mathrm{~d}, 2 \times \mathrm{CH}_{2,} \times \mathrm{Et}, \alpha, \beta, J_{\mathrm{C}, \mathrm{P}}=6.7, J_{\mathrm{C}, \mathrm{P}}=6.2\right)$, 50.6, $50.1(\mathrm{C}-5$ ' $, \alpha, \beta), 24.29,24.26\left(\mathrm{CH}_{2} \mathrm{P}, \alpha, \beta, J_{\mathrm{C}, \mathrm{P}}=142.8\right), 21.3,21.0,20.9,20.6$ $\left(\mathrm{CH}_{3}, \mathrm{Ac}, \alpha, \beta\right), 16.5$ (d, $\left.2 \times \mathrm{CH}_{3}, 2 \times \mathrm{Et}, \alpha, \beta, J_{\mathrm{C}, \mathrm{P}}=6.0\right) .{ }^{31} \mathrm{P} \mathrm{NMR}\left(162 \mathrm{MHz}, \mathrm{CDCl}_{3}\right)$ : $\delta$ 24.79. HRMS: calcd for $\mathrm{C}_{23} \mathrm{H}_{32} \mathrm{~N}_{3} \mathrm{O}_{9} \mathrm{P}[M+\mathrm{H}]^{+}$526.1949, found 526.1959; calcd for $\mathrm{C}_{23} \mathrm{H}_{32} \mathrm{~N}_{3} \mathrm{O}_{9} \mathrm{P}[M+\mathrm{Na}]^{+}$548.1768, found 548.1774. 


\section{1,2-Di- $O$-acetyl-5-deoxy-5-[4-(diethylphosphono)methyl- $1 H$-1,2,3-triazol-1-yl]-3-O- dodecyl- $\alpha$-D-xylofuranose $(43-\alpha, \beta)$}

Obtained according to the general procedure starting from 5-deoxy-5-[4(diethylphosphono)methyl-1H-1,2,3-triazol-1-yl]-3-O-dodecyl-1,2- $O$-isopropylidene- $\alpha$ D-xylofuranose $(40,109 \mathrm{mg}, 0.195 \mathrm{mmol})$. Purification by column chromatography (EtOAc/hexane, 6:1) afforded the title compound (80 mg, 68\%, two steps, anomeric mixture, $\alpha / \beta$ ratio, $1: 0.5)$ as a colourless oil.

${ }^{1} \mathrm{H}$ NMR (400 MHz, $\mathrm{CDCl}_{3}$ ): $\delta 7.63,7.62\left(2 \mathrm{~d}, 1.4 \mathrm{H}, \mathrm{H}-5^{\prime} \alpha, \mathrm{H}-5^{\prime} \beta, J=2.1, J=1.6\right.$ ), $6.44\left(\mathrm{~d}, 1 \mathrm{H}, \mathrm{H}-1^{\prime} \alpha, J_{1^{\prime}, 2^{\prime}(\alpha)}=4.4\right), 6.14\left(\mathrm{~s}, 0.4 \mathrm{H}, \mathrm{H}-1^{\prime} \beta\right), 5.26-5.21$ (m, 1.5 H, H-2' $\alpha, \mathrm{H}-$ $2^{\prime} \beta$ ), 4.81-4.65 (m, 2.8 H, H-5'a $\alpha, \mathrm{H}^{-5}$ 'a $\beta, \mathrm{H}^{-} 4^{\prime} \alpha, \mathrm{H}-4^{\prime} \beta$ ), 4.58-4.49 (m, H-5'b $\beta$ ), 4.44 $\left(\mathrm{dd}, 1 \mathrm{H}\right.$, part B of ABX system, H-5'b $\left.\alpha, J_{4^{\prime}, 5^{\prime} \mathrm{b}(\alpha)}=8.3, J_{5^{\prime} \mathrm{a}, 5^{\prime} \mathrm{b}(\alpha)}=14.1\right), 4.19-4.05(\mathrm{~m}$, $\left.7 \mathrm{H}, \mathrm{H}-3^{\prime} \alpha, \mathrm{H}-3^{\prime} \beta, 2 \times \mathrm{CH}_{2}, 2 \times \mathrm{Et}, \alpha, \beta\right), 3.79-3.59\left(\mathrm{~m}, 1.4 \mathrm{H}, \mathrm{H}-1^{\prime \prime} \mathrm{a}, \alpha, \beta, J_{1 " \mathrm{a}, 2^{\prime \prime a}}=\right.$ $\left.J_{1^{\prime \prime}, 2^{\prime \prime} \mathrm{b}}=6.4, J_{1^{\prime \prime}, 1^{\prime \prime} \mathrm{b}}=9.0\right), 3.54-3.41\left(\mathrm{~m}, 1.4 \mathrm{H}, \mathrm{H}-1^{\prime \prime} \mathrm{b}, \alpha, \beta\right), 3.40-3.25(\mathrm{~m}, 2.8 \mathrm{H}$, $\left.\mathrm{CH}_{2} \mathrm{P}, \alpha, \beta, J_{\mathrm{a}, \mathrm{b}}(\mathrm{CH} 2 \mathrm{P})=19.6, J_{\mathrm{H}-\mathrm{a}, \mathrm{P}}=16.1, J_{\mathrm{H}-\mathrm{b}, \mathrm{P}}=16.1\right), 2.15,2.11,2.06(3 \mathrm{~s}, 5.4 \mathrm{H}$, $\left.\mathrm{CH}_{3}, \mathrm{Ac}, \alpha, \beta\right), 1.63-1.54\left(\mathrm{~m}, 2.8 \mathrm{H}, \mathrm{CH}_{2}-2^{\prime \prime}, \alpha, \beta\right), 1.41-1.21\left(\mathrm{~m}, 33.6 \mathrm{H}, \mathrm{CH}_{2}-3^{\prime \prime}\right.$ to $\left.\mathrm{CH}_{2}-11^{\prime \prime}, 2 \times \mathrm{CH}_{3}, 2 \times \mathrm{Et}, \alpha, \beta\right), 0.88\left(\mathrm{t}, 4.2 \mathrm{H}, \mathrm{CH}_{3}-12^{\prime \prime}, \alpha, \beta, J=6.7\right) .{ }^{13} \mathrm{C}$ NMR (100 $\left.\mathrm{MHz}, \mathrm{CDCl}_{3}\right): \delta 169.9,169.6,169.5,169.2(\mathrm{CO}, \mathrm{Ac}, \alpha, \beta), 138.5\left(\mathrm{~d}, \mathrm{C}-4 \alpha, J_{\mathrm{C}-4, \mathrm{P}(\alpha)}=\right.$ $7.1), 138.4\left(\mathrm{~d}, \mathrm{C}-4 \beta, J_{\mathrm{C}-4, \mathrm{P}(\beta)}=6.6\right), 124.3\left(\mathrm{C}-5 \beta, J_{\mathrm{C}-5, \mathrm{P}(\beta)}=3.9\right), 124.1\left(\mathrm{C}-5 \alpha, J_{\mathrm{C}-5, \mathrm{P}(\alpha)}\right.$ = 4.2), $99.7\left(\mathrm{C}-1^{\prime} \beta\right), 94.0\left(\mathrm{C}-1^{\prime} \alpha\right), 81.4\left(\mathrm{C}-4^{\prime} \beta\right), 80.6,80.1$ (C-3' $\left.\alpha, \mathrm{C}-3^{\prime} \beta\right), 78.9$ (C-2' $\beta), 78.2\left(\mathrm{C}-4^{\prime} \alpha\right), 76.4\left(\mathrm{C}-2^{\prime} \alpha\right), 71.4,70.9\left(\mathrm{CH}_{2}-1^{\prime \prime}, \alpha, \beta\right), 62.51,62.48\left(2 \mathrm{~d}, 2 \times \mathrm{CH}_{2}, 2\right.$ $\left.\times \mathrm{Et}, \alpha, \beta, J_{\mathrm{C}, \mathrm{P}}=6.7, J_{\mathrm{C}, \mathrm{P}}=6.5\right), 51.1,50.1\left(\mathrm{C}-5^{\prime}, \alpha, \beta\right), 32.0,29.8,29.7,29.7,29.6,29.6$, $29.5,29.5,29.4,26.2,26.1\left(\mathrm{C}-2^{\prime \prime}\right.$ to $\left.\mathrm{C}-10^{\prime \prime}, \alpha, \beta\right), 24.18,24.13\left(\mathrm{CH}_{2} \mathrm{P}, \alpha, \beta, J_{\mathrm{C}, \mathrm{P}}=\right.$ 143.0), 22.8 (C-11", $\alpha, \beta), 21.2,20.9,20.8,20.6\left(\mathrm{CH}_{3}, \mathrm{Ac}, \alpha, \beta\right), 16.4\left(\mathrm{~d}, 2 \times \mathrm{CH}_{3}, 2 \times\right.$ Et, $\left.\alpha, \beta, J_{\mathrm{C}, \mathrm{P}}=6.0\right), 14.2\left(\mathrm{C}-12^{\prime \prime}, \alpha, \beta\right) .{ }^{31} \mathrm{P} \mathrm{NMR}\left(162 \mathrm{MHz}, \mathrm{CDCl}_{3}\right): \delta 24.85 . \mathrm{HRMS}$ : calcd for $\mathrm{C}_{28} \mathrm{H}_{50} \mathrm{~N}_{3} \mathrm{O}_{9} \mathrm{P}[\mathrm{M}+\mathrm{H}]^{+} 604.3357$, found 604.3362 ; calcd for $\mathrm{C}_{28} \mathrm{H}_{50} \mathrm{~N}_{3} \mathrm{O}_{9} \mathrm{P}$ $[\mathrm{M}+\mathrm{Na}]^{+} 626.3177$, found 626.3179 .

\section{General procedure for N-glycosylation of uracil with 1,2-O-acetyl xylofuranose 5- (triazolyl)methyl phosphonates}

To a suspension of uracil ( $0.15 \mathrm{mmol}, 2$ equiv. relatively to the glycosyl acetate) in anhydrous acetonitrile $(1.5 \mathrm{~mL}), \mathrm{N}, \mathrm{O}$-bis(trimethylsilyl)acetamide (BSA, 3 equiv.) was added and the mixture was stirred at room temperature for $20 \mathrm{~min}$, whereupon a clear solution of the silylated derivative was obtained. A solution of 1,2-O-acetyl xylofuranose 5-(triazolyl)methyl phosphonate $(0.1 \mathrm{mmol})$ in anhydrous acetonitrile $(1.5$ $\mathrm{mL}$ ) was added to the previous solution, followed by dropwise addition of trimethylsilyl triflate (TMSOTf, 6.5 equiv.). The mixture was stirred under microwave irradiation $(150 \mathrm{~W}, \mathrm{P} \max =250 \mathrm{Psi})$ at $65^{\circ} \mathrm{C}$ for $45 \mathrm{~min}$. It was then diluted with dichloromethane and neutralized with satd. aq. $\mathrm{NaHCO}_{3}$ solution. The aqueous phase was extracted with dichloromethane $(3 \mathrm{x})$ and the combined organic phases were washed with brine and dried with anhydrous $\mathrm{MgSO}_{4}$. After filtration and concentration under vacuum, the residue was subjected to column chromatography. 


\section{1-\{2-O-Acetyl-3-O-benzyl-1,5-dideoxy-5-[4-(diethoxyphosphono)methyl-1H-1,2,3- triazol-1-yl]- $\alpha$-D-xylofuranos-1-yl\}uracil (44)}

Obtained according to the general procedure, starting from 1,2-di- $O$-acetyl-3- $O$-benzyl5-deoxy-5-[4-(diethylphosphono)methyl-1H-1,2,3-triazol-1-yl]- $\alpha, \beta$-D-xylofuranose (42$\alpha, \beta, 50 \mathrm{mg}, 0.095 \mathrm{mmol})$ and uracil $(17 \mathrm{mg}, 0.152 \mathrm{mmol})$ and using BSA (0.07 $\mathrm{mL}$, $0.297 \mathrm{mmol})$ and TMSOTf $(0.12 \mathrm{~mL}, 0.644 \mathrm{mmol})$. Purification by flash column chromatography (EtOAc/MeOH, from 24:1 to 18:1) afforded the title compound (20 $\mathrm{mg}, 36 \%)$ as a colorless oil.

$[\alpha]_{D}^{20}=+6\left(\mathrm{c}=1\right.$, in $\left.\mathrm{CH}_{2} \mathrm{Cl}_{2}\right) .{ }^{1} \mathrm{H} \mathrm{NMR}\left(400 \mathrm{MHz}, \mathrm{CDCl}_{3}\right): \delta 9.21$ (br. s, $\left.1 \mathrm{H} \mathrm{NH}\right)$, 7.65 (d, 1H, H-5", J = 1.7), 7.60 (d, 1H, H-6, $J_{5,6}=8.2$ ), 7.43-7.28 (m, 5 H, Ph), 6.12 (s, $1 \mathrm{H}, \mathrm{H}-1^{\prime}$ ), 5.67 (br.d, $\left.1 \mathrm{H}, \mathrm{H}-5\right), 5.18$ (s, $1 \mathrm{H}, \mathrm{H}-2^{\prime}$ ), 4.78 (d, $1 \mathrm{H}$, part A of AB system, $\mathrm{H}$-a from $\left.\mathrm{CH}_{2} \mathrm{Ph}, \mathrm{Ja}, \mathrm{b}=11.5\right)$, 4.74-4.56 (m, $3 \mathrm{H}, \mathrm{CH}_{2}-5^{\prime}, \mathrm{H}-\mathrm{b}$ from $\mathrm{CH}_{2} \mathrm{Ph}, J_{5^{\prime} \mathrm{a}, 5^{\prime} \mathrm{b}}=$ $\left.14.3, J_{4^{\prime}, 5^{\prime} \mathrm{a}}=5.0, J_{4^{\prime}, 5^{\prime} \mathrm{b}}=7.4\right), 4.50\left(\mathrm{ddd}, 1 \mathrm{H}, \mathrm{H}-4^{\prime}\right), 4.15-4.02\left(\mathrm{~m}, 4 \mathrm{H}, 2 \times \mathrm{CH}_{2}, 2 \times \mathrm{Et}\right)$, $3.83\left(\mathrm{~d}, 1 \mathrm{H}, \mathrm{H}-3^{\prime}, J_{3^{\prime}, 4^{\prime}}=3.1\right), 3.38-3.21\left(\mathrm{~m}, 2 \mathrm{H}, \mathrm{CH}_{2} \mathrm{P}, J_{\mathrm{a}, \mathrm{b}(\mathrm{CH} 2 \mathrm{P})}=19.2, J_{\mathrm{H}-\mathrm{a}, \mathrm{P}}=16.3\right.$, $\left.J_{\mathrm{H}-\mathrm{b}, \mathrm{P}}=16.0\right), 2.13\left(\mathrm{~s}, 3 \mathrm{H}, \mathrm{CH}_{3}, \mathrm{Ac}\right), 1.31-1.21\left(\mathrm{~m}, 6 \mathrm{H}, 2 \times \mathrm{CH}_{3}, 2 \times \mathrm{Et}\right) .{ }^{13} \mathrm{C} \mathrm{NMR}$ (100 MHz, CDCl3): $\delta 169.8$ (CO, Ac), 163.0 (C-4), 150.1 (C-2), 140.1 (C-6), 138.8 (d, $\left.\mathrm{C}-4^{\prime \prime}, J_{\mathrm{C}-4^{\prime \prime}, \mathrm{P}}=6.8\right) 136.0(\mathrm{Cq}, \mathrm{Ph}), 129.0,128.9,128.7(\mathrm{CH}, \mathrm{Ph}), 123.9\left(\mathrm{C}-5^{\prime \prime}, J_{\mathrm{C}-5^{\prime \prime}, \mathrm{P}}=\right.$ 3.8), 103.2 (C-5), $88.9\left(\mathrm{C}-1^{\prime}\right), 80.8$ (C-4'), $79.8\left(\mathrm{C}-3^{\prime}\right), 79.6\left(\mathrm{C}-2^{\prime}\right), 72.2\left(\mathrm{CH}_{2} \mathrm{Ph}\right), 62.61$, $62.57\left(2 \mathrm{~d}, 2 \times \mathrm{CH}_{2}, 2 \times \mathrm{Et}, J_{\mathrm{C}, \mathrm{P}}=6.6\right), 48.9\left(\mathrm{C}-5^{\prime}\right), 24.1\left(\mathrm{CH}_{2} \mathrm{P}, J_{\mathrm{C}, \mathrm{P}}=142.7\right), 20.9$ $\left(\mathrm{CH}_{3}, \mathrm{Ac}\right), 16.5\left(\mathrm{~d}, 2 \times \mathrm{CH}_{3}, 2 \times \mathrm{Et}, J_{\mathrm{C}, \mathrm{P}}=6.0\right) .{ }^{31} \mathrm{P} \mathrm{NMR}(162 \mathrm{MHz}, \mathrm{CDCl} 3): \delta 24.78$. HRMS: calcd for $\mathrm{C}_{25} \mathrm{H}_{32} \mathrm{~N}_{5} \mathrm{O}_{9} \mathrm{P}[\mathrm{M}+\mathrm{H}]^{+}$578.2010, found 578.2011; calcd for $\mathrm{C}_{25} \mathrm{H}_{32} \mathrm{~N}_{5} \mathrm{O}_{9} \mathrm{P}[\mathrm{M}+\mathrm{Na}]^{+} 600.1830$, found 600.1830 .

1-\{2-O-Acetyl-1,5-dideoxy-5-[4-(diethoxyphosphono)methyl-1H-1,2,3-triazol-1-yl]3-O-dodecyl- $\alpha$-D-xylofuranos-1-yl\}uracil (45)

Obtained according to the general procedure, starting from 1,2-di- $O$-acetyl-5-deoxy-5[4-(diethylphosphono)methyl-1 $H$-1,2,3-triazol-1-yl]-3-O-dodecyl- $\alpha$-D-xylofuranose (43- $\alpha, \beta, 45 \mathrm{mg}, 0.074 \mathrm{mmol})$ and uracil $(13 \mathrm{mg}, 0.12 \mathrm{mmol})$ and using BSA (0.06 mL, $0.24 \mathrm{mmol})$ and TMSOTf $(0.09 \mathrm{~mL}, 0.50 \mathrm{mmol})$. Purification by flash column chromatography (EtOAc/MeOH, from 24:1 to 18:1) afforded the title compound (8 $\mathrm{mg}$, $16 \%$ ) as colorless oil.

$[\alpha]_{D}^{20}=+5\left(\mathrm{c}=1\right.$, in $\left.\mathrm{CH}_{2} \mathrm{Cl}_{2}\right) .{ }^{1} \mathrm{H}$ NMR $\left(400 \mathrm{MHz}, \mathrm{CDCl}_{3}\right): \delta 8.69$ (br. s, $\left.1 \mathrm{H} \mathrm{NH}\right)$, 7.70 (d, 1H, H-5", $J=1.7$ ), 7.63 (d, 1H, H-6, $J_{5,6}=8.2$ ), 6.10 (s, 1 H, H-1'), 5.76 (br.d, 1 H, H-5), 5.09 (s, $\left.1 \mathrm{H}, \mathrm{H}-2^{\prime}\right), 4.80\left(\mathrm{~d}, 1 \mathrm{H}\right.$, part A of AB system, H-5'a, $J_{4^{\prime}, 5^{\prime} \mathrm{a}}=4.7, J_{5 \mathrm{a}^{\prime}, 5^{\prime} \mathrm{b}}$ = 14.3), $4.64\left(\mathrm{~d}, 1 \mathrm{H}\right.$, part B of AB system, H-5'b, $\left.J_{4^{\prime}, 5^{\prime} \mathrm{b}}=7.6\right), 4.52$ (ddd, $\left.1 \mathrm{H}, \mathrm{H}-4^{\prime}\right)$, 4.16-4.02 (m, $\left.4 \mathrm{H}, 2 \times \mathrm{CH}_{2}, 2 \times \mathrm{Et}\right), 3.95$ (d, $\left.1 \mathrm{H}, \mathrm{H}-3^{\prime}, J_{3^{\prime}, 4^{\prime}}=3.2\right), 3.74$ (dt, $1 \mathrm{H}, \mathrm{H}-$ $\left.1^{\prime \prime \prime} \mathrm{a}, J_{1^{\prime \prime} \mathrm{a}, 2^{\prime \prime \prime} \mathrm{a}}=J_{1^{\prime \prime \prime}, 2^{\prime \prime \prime} \mathrm{b}}=6.6, J_{1 " \prime \mathrm{a}, 1^{\prime \prime \prime} \mathrm{b}}=9.3\right), 3.52\left(\mathrm{dt}, 1 \mathrm{H}, \mathrm{H}-1^{\prime \prime \prime} \mathrm{b}, J_{1^{\prime \prime \prime} \mathrm{b}, 2^{\prime \prime \prime} \mathrm{a}}=J_{1^{\prime \prime \prime}, 2^{\prime \prime \prime} \mathrm{b}}=\right.$ 6.6), 3.39-3.23 (m, $\left.2 \mathrm{H}, \mathrm{CH}_{2} \mathrm{P}, J_{\mathrm{a}, \mathrm{b}(\mathrm{CH} 2 \mathrm{P})}=19.9, J_{\mathrm{H}-\mathrm{a}, \mathrm{P}}=15.9, J_{\mathrm{H}-\mathrm{b}, \mathrm{P}}=15.8\right), 2.12(\mathrm{~s}, 3 \mathrm{H}$, $\left.\mathrm{CH}_{3}, \mathrm{Ac}\right), 1.63-1.52$ (m, $\left.2 \mathrm{H}, \mathrm{CH}_{2}-2^{\prime \prime \prime}\right), 1.37-1.17$ (m, $24 \mathrm{H}, \mathrm{CH}_{2}-3^{\prime \prime}$ to $\mathrm{CH}_{2}-11^{\prime \prime}, 2 \times$ $\left.\mathrm{CH}_{3}, 2 \times \mathrm{Et}\right), 0.87\left(\mathrm{t}, 3 \mathrm{H}, \mathrm{CH}_{3}-12 ", J=6.9\right) .{ }^{13} \mathrm{C} \mathrm{NMR}(100 \mathrm{MHz}, \mathrm{CDCl} 3): \delta 169.6$ (CO, Ac), 162.7 (C-4), 150.0 (C-2), 140.2 (C-6), 138.8 (C-4"), 123.8 (C-5", J $J_{\mathrm{C}-5^{\prime \prime}, \mathrm{P}}=$ 
4.1), 103.1 (C-5), 89.0 (C-1'), 81.0 (C-4'), 80.9 (C-3'), 79.5 (C-2'), 70.9 (C-1'"'), 62.60, $62.57\left(2 \mathrm{~d}, 2 \times \mathrm{CH}_{2}, 2 \times \mathrm{Et}, J_{\mathrm{C}, \mathrm{P}}=6.5\right), 48.8\left(\mathrm{C}-5^{\prime}\right), 32.0,29.8,29.8,29.7,29.5,29.5$, 26.4 (C-2" to C-10"), 24.2 (d, $\left.\mathrm{CH}_{2} \mathrm{P}, J_{\mathrm{C}, \mathrm{P}}=143.5\right), 22.8\left(\mathrm{C}-11^{\prime \prime}\right), 20.9\left(\mathrm{CH}_{3}, \mathrm{Ac}\right), 16.5$ $\left(\mathrm{d}, 2 \times \mathrm{CH}_{3}, 2 \times \mathrm{Et}, J_{\mathrm{C}, \mathrm{P}}=6.1\right), 14.3\left(\mathrm{C}-12^{\prime \prime}\right) .{ }^{31} \mathrm{P} \mathrm{NMR}\left(162 \mathrm{MHz}, \mathrm{CDCl}_{3}\right): \delta 24.79$. HRMS: calcd for $\mathrm{C}_{30} \mathrm{H}_{50} \mathrm{~N}_{5} \mathrm{O}_{9} \mathrm{P}[M+\mathrm{H}]^{+}$656.3419, found 656.3434; calcd for $\mathrm{C}_{30} \mathrm{H}_{50} \mathrm{~N}_{5} \mathrm{O}_{9} \mathrm{P}[M+\mathrm{Na}]^{+}$678.3238, found 678.3254.

\section{Crystal structure determination}

X-Ray diffraction data were collected at $296 \mathrm{~K}$ on a Bruker AXS-KAPPA D8 QUEST diffractometer using graphite monochromated Mo-K $\alpha$ radiation $(\lambda=0.71073 \AA)$.

All nonhydrogen atoms were refined anisotropically whereas the hydrogen atoms were placed in calculated positions and allowed to refine riding on the parent atom on compounds 12-a and 20 while in compound 35 were located from the electron density map and allowed to refine freely. All the structures were solved by direct methods with Bruker SHELXTL ${ }^{34}$ and refined by full-matrix least-squares on $F^{2}$ using SHELXL $2017 / 1,{ }^{35}$ both programs included in WINGX version $2018.3{ }^{36}$ Graphics were made with MERCURY 4.3.1 ${ }^{37}$ and PLATON ${ }^{38}$ was used for determination of hydrogen bond interactions.

Crystallographic data for the compounds 12-a, 20 and 35 were deposited at the Cambridge Crystallographic Data Centre under the CCDC numbers 2004694, 2004693 and 2004692, respectively.

Data for 12-a: Colourless monocrystals for X-ray studies were obtained from crystallization with ethyl acetate. Crystal data: orthorhombic; space group P $2_{1} 2_{1} 2_{1}$, a = 5.4925(4), $\mathrm{b}=10.0614(8), \mathrm{c}=21.3348(16) \AA ; \mathrm{V}=1179.01(15) \AA^{3}, \mathrm{Z}=4, \mu=0.110$ $\mathrm{mm}^{-1}$. A total of 10880 reflection intensities were collected up to $2 \theta_{\max }=25^{\circ}$; for structure refinement 2583 independent reflections with $\mathrm{I}>4 \sigma(\mathrm{I})$ were used. The final $\mathrm{R}_{\text {factor }}$ was 0.0317 and $\mathrm{Rw}=0.0705$.

Data for 20: Colourless monocrystals for X-ray studies were obtained. Crystal data: orthorhombic; $a=8.8217(16), b=11.0251(18), c=19.290(3) \AA ; \quad V=1876.2(5) \AA^{3}, Z=$ $4, \mu=0.110 \mathrm{~mm}^{-1}$; space group was P 212121 . A total of 7630 reflection intensities were collected up to $2 \theta_{\max }=21^{\circ}$; for structure refinement 3473 independent reflections with $\mathrm{I}>4 \sigma(\mathrm{I})$ were used. The final $\mathrm{R}_{\text {factor }}$ was 0.0390 and $\mathrm{Rw}=0.0811$.

Data for 35: Colourless monocrystals for X-ray studies were obtained. Crystal data: monoclinic; $\mathrm{a}=5.1766(5), \mathrm{b}=10.7265(13), \mathrm{c}=16.464(2) \AA ; \mathrm{V}=913.99(18) \AA^{3}, \mathrm{Z}=2$, $\mu=0.110 \mathrm{~mm}^{-1}$; space group was P 21. A total of 6749 reflection intensities were collected up to $2 \theta_{\max }=25^{\circ}$; for structure refinement 2583 independent reflections with I $>4 \sigma(\mathrm{I})$ were used. The final $\mathrm{R}_{\text {factor }}$ was 0.0372 and $\mathrm{Rw}=0.0753$. 


\section{Computational Details}

All DFT calculations were perfomed with Gaussian 09. ${ }^{31}$ Using three-dimensional structures of 12-a and 12-b built using Pymol, ${ }^{30}$ the geometries were optimized using the PBE1PBE functional, also known as PBE0, ${ }^{39}$ along with the standard 6-311G** basis set for all elements. Solvent effects (toluene or chloroform) were taken into account using a polarizable continuum model described by the SMD variation of the integral equation formalism variant (IEFPCM), ${ }^{40}$ as implemented in Gaussian09. Frequency calculations were performed to characterize the stationary points, all corresponding to true minima. The optimized structures of 12-a (toluene, chloroform) were compared with the experimental X-ray structure with Pymol by pair-fitting the non-hydrogen atoms, yielding RMSD values of $0.093 \AA$ and $0.096 \AA$, respectively.

\section{Conflicts of interest}

There are no conflicts to declare.

\section{Acknowledgments}

The authors thank 'Fundacão para a Ciência e Tecnologia' (FCT) for funding through the FCT Investigator Program - grant numbers IF/01488/2013 (N.M.X) and IF/00069/2014 (P.J.C), the exploratory projects IF/01488/2013/CP1159/CT0006 (N.M.X.) and IF/00069/2014/CP1216/CT0006 (P.J.C.), and the projects UIDB/00100/2020, UIDP/00100/2020 (CQE), UID/MULTI/04046/2019, UIDB/04046/2020, UIDP/04046/2020 (BioISI) and UID/MULTI/00612/2013, UID/MULTI/00612/2019 (Centro de Química e Bioquímica). Financial support from FCT/MCTES, Programa Operacional Regional de Lisboa (Lisboa 2020), Portugal 2020, FEDER/FN, and the European Union under Project No. 28455 (LISBOA-01-0145FEDER-028455, PTDC/QUI-QFI/28455/2017) is also acknowledged. 


\section{References}

[1] (a) L. P. Jordheim, D. Durantel, F. Zoulim and C. Dumontet, Nat. Rev. Drug Discov., 2013, 12, 447-464; (b) J. Shelton, X. Lu, J. A. Hollenbaugh, J. H. Cho, F. Amblard, R. F. Schinazi, Chem. Rev. 2016, 116, 14379-14455; (c) W. B. Parker, Chem. Rev. 2009, 109, 2880-2893.

[2] (a) V. L. Damaraju, S. Damaraju, J. D. Young, S. A. Baldwin, J. Mackey, M. B. Sawyer, C. E. Cass, Oncogene 2003, 22, 7524-7536; (b) J. Zhang, F. Visser, K. M. King, S. A. Baldwin, J. D. Young, C. E. Cass, Cancer Metastasis Rev. 2007, 26, 85110; (c) K. D. Ibarra, J. K. Pfeiffer, J. Virol. 2009, 83, 4538-4547.

[3] (a) C. M. Galmarini, M. L. Clarke, L. Jordheim, C. L. Santos, E. Cros, J. R. Mackey, C. Dumontet, BMC Pharmacol. 2004, 4, 8; (b) O. H. Temmink, I. V. Bijnsdorp, H.-J. Prins, N. Losekoot, A. D. Adema, K. Smid, R. J. Honeywell, B. Ylstra, P. P. Eijk, M. Fukushima, G. J. Peters, Mol Cancer Ther. 2010, 9, 1047-1057; (c) M. I. Besecker, C. L. Furness, D.M. Coen, A.Griffiths, J. Virol. 2007, 81, 8356-8360.

[4] (a) M. S. Patzak, V. Kari, S. Patil, F. H. Hamdan, R. G. Goetze, M. Brunner, J. Gaedcke, J. Kitz, D. I. Jodrell, F. M. Richards, C. Pilarsky, R.Gruetzmann, P. Rümmele, T. Knösel, E. Hessmann, V. Ellenrieder, S. A. Johnsen, A. Neesse, EBioMedicine 2019, 40, 394-405; (b) S. A. Hunsucker, J. Spychala, B. S. Mitchell, J. Biol. Chem. 2001, 276, 10498-10504.

[5] (a) E. De Clercq, A, Holý, Nat Rev Drug Discov. 2005, 4, 928-940; (b) E. De Clercq, Biochem. Pharmacol. 2011, 82, 99-109; (c) M.-S. Xie, H.-Y. Niu, G.-R. Qu, H.M. Guo, Tetrahedron Lett. 2014, 55, 7156-7166.

[6] (a) L. S. Jeong, J. A. Lee, Antiviral Chem. Chemother. 2004, 15, 235-250; (b) M. Bessières, F. Chevrier, V. Roy, L. A. Agrofoglio, Future Med. Chem. 2015, 7, 18091828.

[7] G. Romeo, U. Chiacchio, A. Corsaro, P. Merino, Chem. Rev. 2010, 110, 3337-3370.

[8] N. M. Xavier, A. Porcheron, D. Batista, R. Jorda, E. Řezníčková, V. Kryštof, M. C. Oliveira, Org. Biomol. Chem. 2017, 15, 4667-4680; N. M. Xavier, R. GonçalvesPereira, R. Jorda, D. Hendrychová, M. C. Oliveira, Pure Appl. Chem. 2019, 91, 10851105 .

[9] (a) J. Štambaský, M. Hocek, P. Kočovský, Chem. Rev. 2009, 109, 6729-6764; (b) E. De Clercq, J. Med. Chem. 2016, 59, 2301-2311; (b) K. Temburnikar, K. L. SeleyRadtke, Beilstein J. Org. Chem. 2018, 14, 772-785. 
[10] C.R. Wagner, V. V. Iyer, E. J. McIntee, Med. Res. Rev. 2000, 20, 417-451.

[11] (a) U. Pradere, E. C. Garnier-Amblard, S. J. Coats, F. Amblard, R. F. Schinazi, Chem. Rev. 2014, 114, 9154-9218; (b) N. M. Xavier, in Elsevier Reference Module in Chemistry, Molecular Sciences and Chemical Engineering, ed. J. Reedijk, Elsevier, Waltham, MA, 2017, pp. 1-15. DOI: 10.1016/B978-0-12-409547-2.12655-1.

[12] (a) M. Winn, R. J. M. Goss, K.-i. Kimura and T. D. H. Bugg, Nat. Prod. Rep., 2010, 27, 279-304; (b) M. Serpi, V. Ferrari and F. Pertusati, J. Med. Chem., 2016, 59, $10343-10382$.

[13] (a) S. Schwarz, R. Csuk and A. P. Rauter, Org. Biomol. Chem., 2014, 12, 24462456; (b) N. M. Xavier, S. Schwarz, P. D. Vaz, R. Csuk and A. P. Rauter, Eur. J. Org. Chem., 2014, 2014, 2770-2779; (c) N. M. Xavier, S. D. Lucas, R. Jorda, S. Schwarz, A. Loesche, R. Csuk, M. C. Oliveira, Synlett 2015, 26, 2663-2672; (d) D. Batista, S. Schwarz, A. Loesche, R. Csuk, P. J. Costa, M. C. Oliveira, N. M. Xavier, Pure Appl. Chem. 2016, 88, 363-379; (e) R. Gonçalves-Pereira, M. P. Pereira, S. G. Serra, A. Loesche, R. Csuk, S. Silvestre, P. J. Costa, M. C. Oliveira, N. M. Xavier, Eur. J. Org. Chem. 2018, 2018, 2667-2681; (f) N. M. Xavier, E. C. de Sousa, M. P. Pereira, A. Loesche, I. Serbian, R. Csuk, M. C. Oliveira, Pharmaceuticals 2019, 12, 103.

[14] (a) H.-W. Yu, L.-R. Zhang, J.-C. Zhuo, L.-T. Ma, L.-H. Zhang, Bioorg. Med. Chem. 1996, 4, 609-614; (b) H.-W.Yu, H.-Y. Zhang, Z.-J. Yang, J.-M. Min, L.-T. Ma, L.-H. Zhang, Pure Appl. Chem. 1998, 70, 435-438.

[15] (a) D. M. Huryn, B. C. Sluboski, S. Y. Tam, M. Weigele, I. Sim, B. D. Anderson, H. Mitsuya, S. Broder, J. Med. Chem. 1992, 35, 2347-2354; (b) J. A. Tino, J. M. Clark, A. K. Field, G. A. Jacobs, K. A. Lis, T. L. Michalik, B. McGeever-Rubin, W. A. Slusarchyk, S. H. Spergel, J. Med. Chem. 1993, 36, 1221-1229; (c) K. F. Solke, J.-L. Huang, J. W. Russell, V. J. Whiterock, J. E. Sundeen, L. W. Stratton, J. M. Clark, Antiviral Res. 1994, 23, 219-224; (d) V. Nair, D. G. Piotrowska, M. Okello, J. Vadakkan, Nucleosides Nucleotides Nucleic Acids 2007, 26, 687-690.

[16] S. G. Agalave, S. R. Maujan, V. S. Pore, Chem. Asian J. 2011, 6, $2696-2718$.

[17] (a) R. Huisgen, G, Szeimies, L. Möbius Chem. Ber. 1967, 100, 2494-2507; (b) V.V. Rostovtsev, L.G. Green, V.V. Fokin, K.B. Sharpless, Angew. Chem., Int. Ed. 2002, 41, 2596-2599; (c) V. D. Bock, H. Hiemstra, J. H. van Maarseveen. Eur. J. Org. Chem. 2006, 2006, 51-68; M. Meldal, C. W. Tornøe, Chem. Rev. 2008, 108, 29523015; (d) L. Liang, D. Astruc, Coord. Chem. Rev. 2011, 255, 2933-2945; (e) For a review on the use of Huisgen azide-alkyne cycloaddition reactions in nucleos(t)ide 
chemistry, see: F. Amblard, J. H. Cho, R. F. Schinazi, Chem. Rev. 2009, 109, 42074220 .

[18] (a) M. L. G. Ferreira, L. C. S. Pinheiro, O. A. Santos-Filho, M. D. S. Peçanha, C. Q. Sacramento, V. Machado, V. F. Ferreira, T. M. L. Souza, N. Boechat, Med. Chem. Res. 2014, 23, 1501-1511; (b) S. K. V. Vernekar, L. Qiu, J. Zhang, J. Kankanala, H. Li, R.J. Geraghty, Z. Wang, J. Med. Chem. 2015, 58, 4016-4028.

[19] (a) J.-L. Yu, Q.-P. Wu, Q.-S. Zhang, Y.-H Liu, Y.-Z. Li, Z.- M. Zhou, Bioorg. Med. Chem. Lett., 2010, 20, 240-243; (b) T. Ostrowski, P. Januszczyk, M. Cieslak, J. Kazmierczak-Baranska, B. Nawrot, E. Bartoszak-Adamska, J. Zeidler, Bioorg. Med. Chem., 2011, 19, 4386-4398; (c) M. Driowya, A. Puissant, G. Robert, P. Auberger, R. Benhida, K. Bougrin, Ultrason. Sonochem., 2012, 19, 1132-1138.

[20] B.Hu, H. Zhao, Z. Chen, C. Xu, J. Zhao, W. Zhao, Molecules 2018, 23, 709.

[21] (a) S. Van Poecke, A. Negri, F. Gago, I. Van Daele, N. Solaroli, A. Karlsson, J. Balzarini, S. Van Calenbergh, J. Med. Chem., 2010, 53, 2902-2912; (b) V. Parmenopoulou, D. S.M. Chatzileontiadou, S. Manta, S. Bougiatioti, P. Maragozidis, D.-N. Gkaragkouni, E.Kaffesaki, A. L. Kantsadi, V. T. Skamnaki, S. E. Zographos b , P. Zounpoulakis, N.A.A. Balatsos, D. Komiotis, D. D. Leonidas, Bioorg. Med. Chem. 2012, 20, 7184-7193; (c) T. N. Van, A. Hospital, C. Lionne, L. P. Jordheim, C. Dumontet, C. Périgaud, L. Chaloin, S. Peyrottes, Beilstein J Org Chem. 2016, 12, 14761486; (d) M. Kozarski, D. Kubacka, B. A. Wojtczak, R. Kasprzyk, M. R. Baranowski, J. Kowalska, Bioorg. Med. Chem. 2018, 26, 191-199.

[22] B. L. Wilkinson, H. Long, E. Sim, A. J. Fairbanks, Bioorg. Med. Chem. Lett. 2008, $18,6265-6267$.

[23] H. Isobe, T. Fujino, N. Yamazaki, M. Guillot-Nieckowski, E. Nakamura, Org. Lett., 2008, 10, 3729-3732.

[24] A. S. Rowan, N. I. Nicely, N. Cochrane, W. A. Wlassoff, A. Claiborne, C. J. Hamilton, Org. Biomol. Chem., 2009, 7, 4029-4036.

[25] A. S. Thompson, G. R. Humphrey, A. M. DeMarco, D. J. Mathre, E. J. J. Grabowski, J. Org. Chem. 1993, 58, 5886-5888.

[26] D. B. Tulshian, A. F. Fundes, M. Czarniecki, Bioorg. Med.Chem. Lett. 1992, 2, $515-518$.

[27] S. Hotha, R. I. Anegundi, A. A. Natu, Tetrahedron Lett. 2005, 46, 4585-4588. 
[28] X. Creary, A. Anderson, C. Brophy, F. Crowell, Z. Funk, J. Org. Chem. 2012, 77, 8756-8761.

[29] R. I. Anegundi, V. G. Puranik, S. Hotha, Org. Biomol. Chem. 2008, 6, 779-786.

[30] PyMOL Molecular Graphics System, Version 1.2r2, DeLano Scientific LLC, San Carlos, CA (USA), 2009.

[31] Gaussian 09, M. J. Frisch, G. W. Trucks, H. B. Schlegel, G. E. Scuseria, M. A. Robb, J. R. Cheeseman, G. Scalmani, V. Barone, G. A. Petersson, H. Nakatsuji, X. Li, M. Caricato, A. Marenich, J. Bloino, B. G. Janesko, R. Gomperts, B. Mennucci, H. P. Hratchian, J. V. Ortiz, A. F. Izmaylov, J. L. Sonnenberg, D. Williams-Young, F. Ding, F. Lipparini, F. Egidi, J. Goings, B. Peng, A. Petrone, T. Henderson, D. Ranasinghe, V. G. Zakrzewski, J. Gao, N. Rega, G. Zheng, W. Liang, M. Hada, M. Ehara, K. Toyota, R. Fukuda, J. Hasegawa, M. Ishida, T. Nakajima, Y. Honda, O. Kitao, H. Nakai, T. Vreven, K. Throssell, J. A. Montgomery Jr., J. E. Peralta, F. Ogliaro, M. Bearpark, J. J. Heyd, E. Brothers, K. N. Kudin, V. N. Staroverov, T. Keith, R. Kobayashi, J. Normand, K. Raghavachari, A. Rendell, J. C. Burant, S. S. Iyengar, J. Tomasi, M. Cossi, J. M. Millam, M. Klene, C. Adamo, R. Cammi, J. W. Ochterski, R. L. Martin, K. Morokuma, O. Farkas, J. B. Foresman, and D. J. Fox, Gaussian, Inc., Wallingford CT, 2016] at the PBE1PBE/6-31G* level of theory [C. Adamo, V. Barone, J. Chem. Phys. 1999, 110, 6158-6169.

[32] R. K. Harris, E. D. Becker, S. M. Cabral de Menezes, R. Goodfellow, P. Granger, Pure Appl. Chem., 2001, 73, 1795-1818.

[33] N. M. Xavier, R. G. Pereira, R. Jorda, E. Řezničkova, V. Kryštof, M. C. Oliveira, Pure Appl. Chem. 2017, 89, 1267-1281.

[34] G. M. Sheldrick, Acta Crystallogr., Sect. A: Found. Adv. 2015, 71, 3-8.

[35] G. M. Sheldrick, Acta Crystallogr., Sect. C: Struct. Chem. 2015, 71, 3-8.

[36] L. J. Farrugia, J. Appl. Crystallogr. 1999, 32, 837-838.

[37] C. F. Macrae, I. Sovago, S. J. Cottrell, P. T. A. Galek, P. McCabe, E. Pidcock, M. Platings, G. P. Shields, J. S. Stevens, M. Towler, P. A. Wood, J. Appl. Cryst., 2020, 53, 226-235.

[38] A. L. Spek, J. Appl. Crystallogr. 2003, 36, 7-13.

[39] C. Adamo, V. Barone, J. Chem. Phys. 1999, 110, 6158-6169. 
[40] A. V. Marenich, C. J. Cramer, and D. G. Truhlar, J. Phys. Chem. B, 2009, 113, 6378-6396. 